\title{
A PALEOECOLOGIC RECONSTRUCTION OF THE HISTORY OF FEATHERBED BANK, BISCAYNE NATIONAL PARK, BISCAYNE BAY, FLORIDA
}

\author{
By \\ Jeffery R. Stone, Thomas M. Cronin, G. Lynn Brewster-Wingard, Scott E. \\ Ishman, Bruce R. Wardlaw, and Charles W. Holmes \\ United States Geological Survey, Reston, VA 20192
}

\section{Open-File Report 00-191}

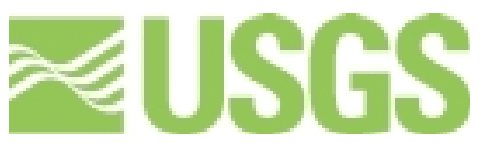

This report is preliminary and has not been reviewed for conformity with

U.S. Geological Survey editorial standards or with the North American Stratigraphic Code. Any use of trade, product, or firm names is for descriptive purposes only and does not imply endorsement by the U.S.

Government 


\section{ABSTRACT}

Using multiple-proxy biological indicators, a paleoecological history of the past 550 years of Featherbed Bank, Biscayne Bay, has been reconstructed from a short (2.26 m) sediment core. Paleoecological changes in ostracode, mollusc, and foraminifer assemblages show that core SEI297-FB-1 can be divided into three distinctly different zones, which together provide evidence for distinct changes in historical environmental conditions at Featherbed Bank.

Assemblages from fossil biotic communities within zone 1, representing approximately 1440 to $1550 \mathrm{AD}$, are characterized by open-marine biota with relatively limited numbers of epiphytic biota. Molluscan faunal indicators suggest the sediment was capable of supporting infaunal organisms and that faunal richness was relatively limited during this time period.

A change in the biotic community occurred around 1550 AD and continued until the late 1800's distinguishing zone 2. Fossil biotic indicators from zone 2 show a strong dominance of epiphytic organisms within all of the biotic communities examined. Foraminifers, molluscs, and ostracodes capable of subsisting in salinities slightly lower than normal marine begin to flourish in this time period, and there is a marked decline in infaunal molluscs.

Zone 2 assemblages are replaced around 1900 AD by increased numbers of organisms that typify open-marine conditions and a return to decreased epiphytic assemblages, similar to zone 1. Zone 3 assemblages, however, show some strong dissimilarities from zone 1, including limited infaunal molluscs, increased abundances of the ostracode $M$ alzella floridana, and a significant increase in molluscan faunal richness. 


\section{ACKNOWLED GEMENTS}

We wish to thank GeneShinn, Don Hickey, Chris Reich, and Marci Marot (USGS, St. Petersburg) for assisting in the collection and processing of Core SEI297-FB-1. A special thank you goes to Biscayne National Park for providing permits, which allowed for the collection of this core within the Park as well as data for modern analogues. We would also like to thank Dade-M etro County Department of Environmental Management. Nancy Carlin (USGS, Reston), helped with processing the core, and Jill D'A mbrosio, Ellen Seefelt, and Ian Graham (USGS, Reston) were responsible for picking the ostracodes and foraminifers used in this study. John M cGeehin provided analysis of the ${ }^{14} \mathrm{C}$ dates, and Sara Schwede assisted in producing an age model. This manuscript benefited from the thorough reviews provided by Laurel Bybell and Karlyn Westover (USGS, Reston). 


\section{TABLE OF CONTENTS}

\section{INTRODUCTION}

Ecosystems and Ecosystem History 1

Setting 2

Multiple-proxy Approach 2

METHODS

Core Collection 5

Core processing 6

Chronology $\quad 6$

\section{RESULTS}

O stracodes 9

Molluscs 11

Foraminifers $\quad 14$

DISCUSSION

Regional Model for Featherbed Bank 17

Bank M igration Model $\quad 19$

$\begin{array}{ll}\text { Suggestions for Future W ork } & 19\end{array}$

CONCLUSIONS

REFERENCES 


\section{LIST OF FIG URES}

Fig. 1: The location of Featherbed Bank within Biscayne N ational Park, Florida. The dot indicates the location where core SEI297-FB-1 was taken in February 1997.

Fig. 2: D escription of core SEI 297-FB-1 (M odified from Ishman, 1997).

Fig. 3: Age M odel for core SEI 297-FB-1.

Fig. 4: Ostracodes (in percent abundance) from Featherbed Bank Core, Biscayne Bay.

Fig. 5: M olluscs (in percent abundance) from Featherbed Bank Core, Biscayne Bay.

Fig. 6: Foraminifers (in percent abundance) from Featherbed Bank Core, Biscayne Bay.

Fig. 7: SEI 297-FB-1 key down-core indicators plotted against time. 


\section{INTRODUCTION}

\section{Ecosystems and Ecosystem History}

Coastal marine environments may change in response to both natural and anthropogenic forces. $N$ atural forces, such as seasonal rainfall variation, hurricanes, climatic variation, and sea-level fluctuation, can dramatically affect substrate and water quality for organisms living within an ecosystem. Human alteration of an environment, such as urbanization, modification of the flow of water and circulation patterns, dredging of natural channels, and introduction of additional nutrients, affect the stability and natural variability of an ecosystem. As a result of tolerances, preferences, and ecological balance, change in the conditions of an ecosystem will result in a concomitant change in biotic assemblages. Therefore, using biota as proxies, historical environmental conditions can be reconstructed from fossil assemblages preserved in short sediment cores.

In 1994, the Everglades Forever Act was passed to help stem the effects of anthropogenic influences upon the Florida Everglades, Florida Bay, and Biscayne Bay. Increasing evidence of changes in the ecosystem attributed to anthropogenic forces, such as industrial pollution, over-harvesting of fisheries, and alteration of natural water circulation patterns, sparked an effort to restore the environment to a more natural condition. However, the impact of natural hydrological and climatic forces had not been adequately investigated. The 'Ecosystem History of Biscayne Bay and the Southeast Coast' project of the USGS was designed to help water management agencies with decisions regarding water and land-use management in south Florida by producing a model for recognizing humaninduced and natural ecosystem variation using biological proxies. Documenting and correlating historical trends in the ecosystem's variation with the timing of man-made, hydrological, and climatic events can be used to help determine what 
aspect of the ecosystem has been affected and help distinguish between the factors influencing environmental change.

\section{Setting}

Featherbed Bank is located on the margin between central Biscayne Bay and southern Biscayne Bay, off the western coast of Sands Key (fig. 1). The Bank is composed of Holocene sediments that may be greater than 4 meters thick in areas and may represent more than 5000 years of sediment accumulation (Wanless and others, 1995). M onthly salinity readings from sites near Featherbed Bank ranged from 30 to 43.2 ppt between July 1988 and December 1998, averaging around 35.4 ppt (Metro-Dade Department of Environmental Resources Management monthly monitoring of Biscayne Bay, unpublished data).

\section{Multiple-proxy Approach}

Ostracodes, foraminifers, and molluscs are small calcareous organisms whose fossil assemblages and shell chemical composition are commonly used in quantitative paleoclimatic reconstruction in deep-sea, coastal, estuarine, and lacustrine environments. These organisms are a major part of the benthic community in Biscayne and Florida Bay, with more than 50 ostracode species, 70 foraminifer species, and over 240 molluscan species (Lyons, 1999). Several studies in Florida Bay and Biscayne Bay have been published that use these biota as a proxy to show changes in a variety of environmental conditions (Turney and Perkins, 1972; Lyons, 1996; Bock and others, 1971; Lidz and Rose, 1989). A nalyses of modern flora and fauna within Biscayne Bay (Ishman and others, 1998; Ishman and D'A mbrosio, 1997; Ishman, in press) and the surrounding environments (Brewster-Wingard and Ishman, 1999; Brewster-Wingard and others, 1996; Brewster-Wingard, Stone, \& Holmes, in press) have been used to help cal ibrate modern analogues for forami nifer, mollusc, and ostracode assemblages. In the present study, a multiple-proxy approach was taken to reconstruct the 
paleoenvironments in order to provide a preliminary interpretation for the last 500 years of Featherbed Bank's history. 


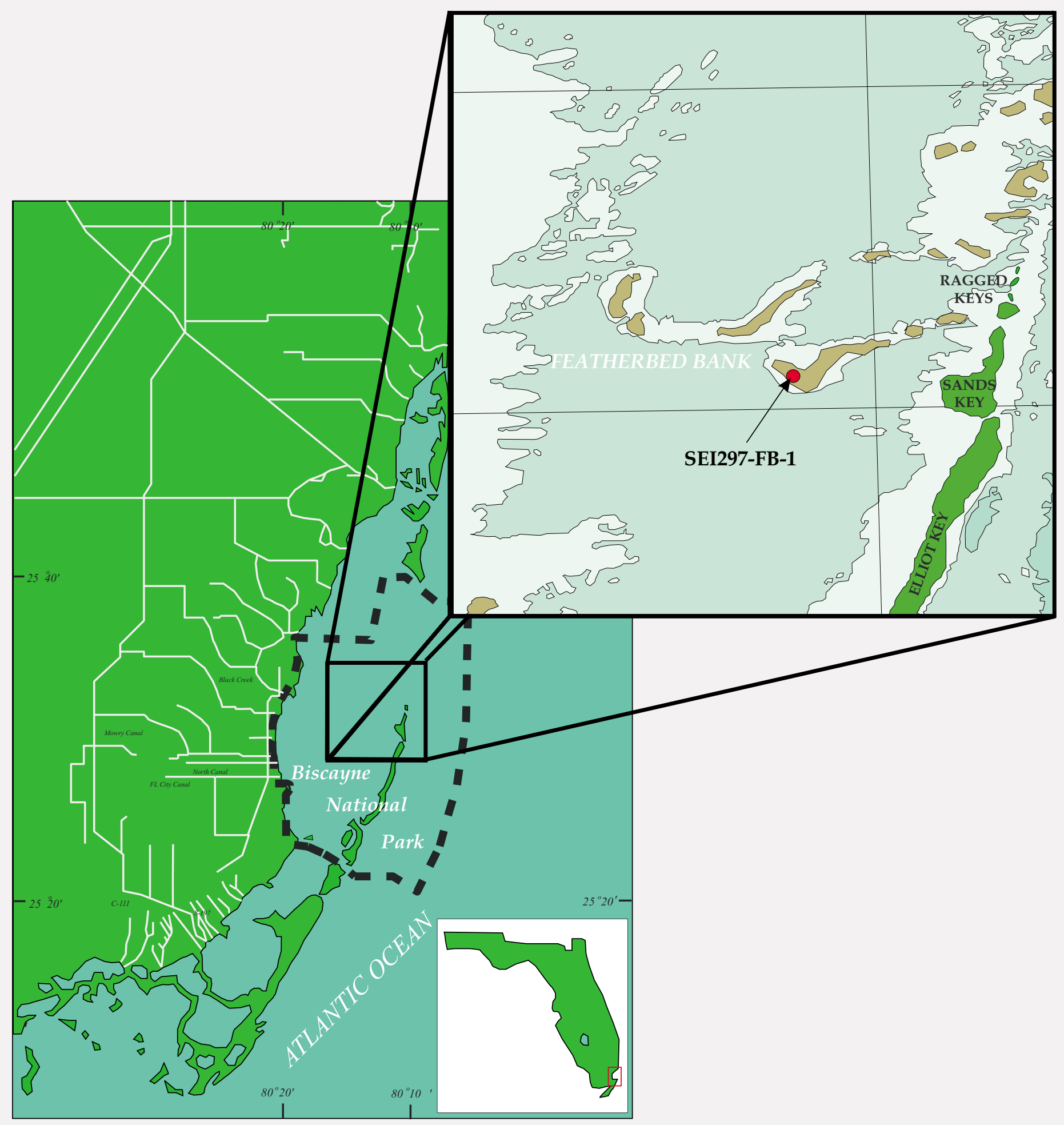

Figure 1. The location of Featherbed Bank within Biscayne National Park, Florida. The dot indicates the location where core SEI297-FB-1 was taken in February 1997. 


\section{METHODS}

\section{Core Collection}

Core SEI297-FB-1, a $2.26 \mathrm{~m}$ long sediment core $\left(25^{\circ} 31.31\right.$ 'N, $\left.80^{\circ} 15.39^{\prime} \mathrm{W}\right)$, was collected in February 1997 using a large (approximately 4 inches in diameter) piston core. The base of the core did not reach the underlying limestone bedrock (Ishman, 1997). The core was collected in water $63 \mathrm{~cm}$ deep,

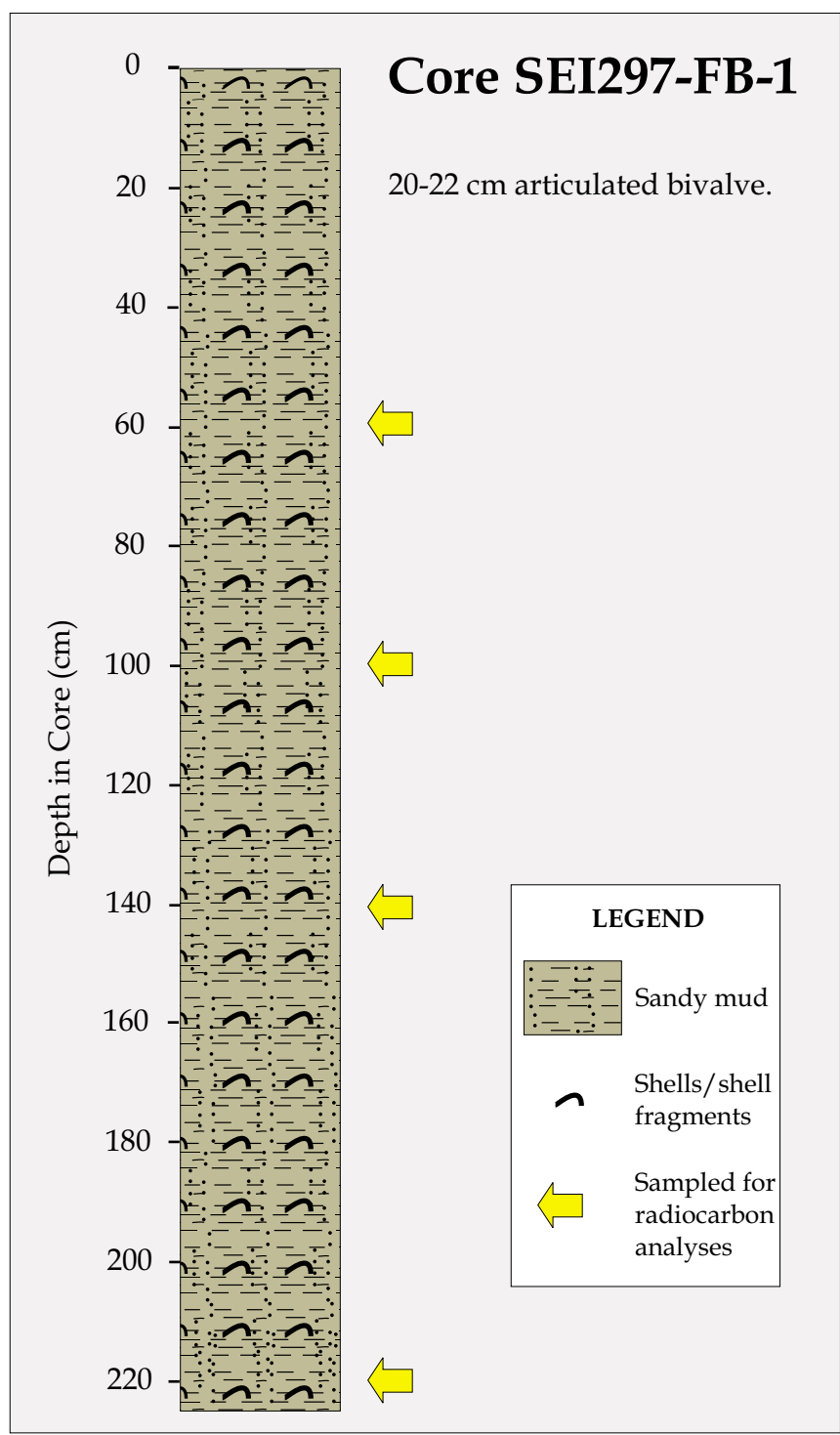

Figure 2. Description of core SEI297-FB-1

(Modified from Ishman, 1997). using the USGS pontoon barge.

A tripod suspended the core tube through a moon pool in the center of the barge. A piston, placed at the surfacewater interface within the core tube, was used to maintain a vacuum that prevented compaction of the sediments as the core tube was being inserted into the sediment. Steel cables attached to the tripod were used to hoist the core tube out of the sediment when the core tube could no longer penetrate the sediment. The recovered core tube was then capped to prevent loss of sediment and to maintain stability in the upper portion of the core before processing (Ishman, 1997). The core 
composition is primarily a homogeneous, light-olivegray, fine sandy mud with plant debris and shell material (fig. 2).

\section{Core Processing}

The core was sampled every 2 centimeters, from the top of the core to the base, for faunal and geochemical analysis. All samples were washed through a set of nested $63 \mu \mathrm{m}$ and $850 \mu \mathrm{m}$ sieves. Sample components from less than $63 \mu \mathrm{m}$ were dried at $50^{\circ} \mathrm{C}$ and weighed for ${ }^{210} \mathrm{~Pb}$ analyses. The fractions of the sample greater than $63 \mu \mathrm{m}$ were dried at $50^{\circ} \mathrm{C}$ and analyzed for ostracodes, foraminifers, and molluscs. All identifiable molluscs (ranging between 5 and 184 specimens), between 97 and 152 ostracode specimens, and 300 foraminifers (when attainable) were picked from each sample with a fine brush. Samples yielding less than 300 foraminifers were picked in their entirety.

Every other sample from the core was examined. Faunal groups and species were identified, counted, and standardized by calculating percent abundance within each sample. Ostracode species were identified using the taxonomy of Teeter (1975), Keyser (1975, 1976, 1977), and Garbett and M addocks (1978). Cronin and others (in press) reviewed the ecology of ostracode species. Molluscs were identified primarily using A bbott (1974), Warmke and A bbott (1961), Perry and Schwengel (1955), and Andrews (1971), and taxonomic nomenclature was updated following Turgeon and others (1998). Taxonomy of the benthic foraminiferal species were identified using Loeblich and Tappan (1988). Faunal slides are housed in the Eastern Earth Surface Processes Team, U.S. Geological Survey, Reston, Virginia.

\section{Chronology}

A provisional age model (fig. 3) for SEl297-FB-1 was developed from 210Pb (lead) dating for the uppermost $90 \mathrm{~cm}$ (Robbins and others, in press), and ${ }^{14} \mathrm{C}$ (radiocarbon) dating by John M cGeehin (USGS, Reston) for the interval from 60 


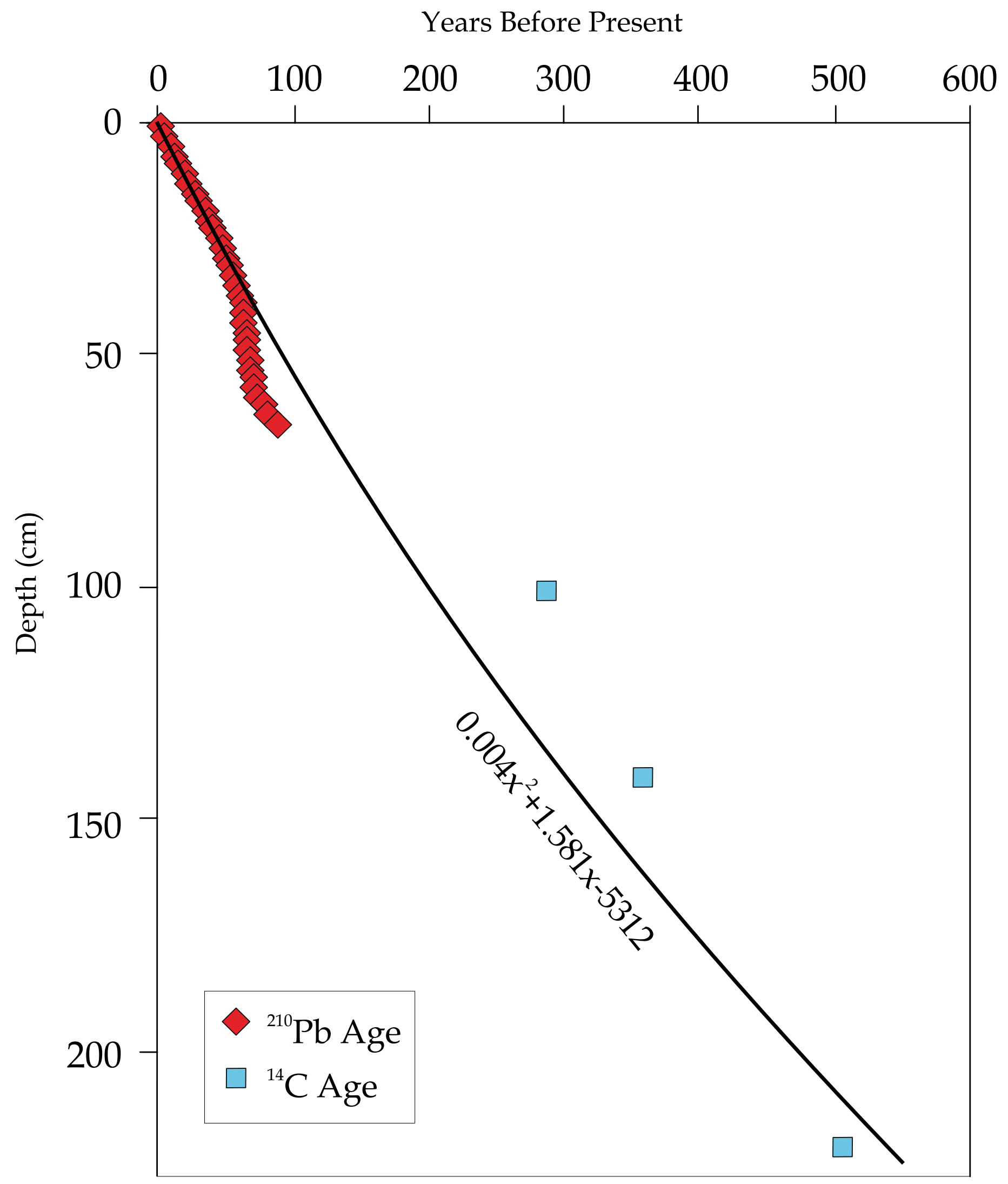

Figure 3. Age model for core SEI297-FB-1. 
to $222 \mathrm{~cm}$ (table 1). Using the ${ }^{210} \mathrm{~Pb}$ ages and lower three radiocarbon dates (101, 141 , and $221 \mathrm{~cm}$ ) as tie-points (fig. 2), a preliminary age model was obtained with a second order polynomial line yielding the following depth age relationship:

$$
\text { Age }=0.004\left(\text { depth }^{2}\right)+1.581(\text { depth })-5.312\left(r^{2}=0.944\right)
$$

Ages for each faunal sample were computed using this equation and are given in Tables 2 - 4.

One can obtain a slightly different age model by using the original age estimates from the ${ }^{210} \mathrm{~Pb}$ analyses for the upper $87 \mathrm{~cm}$ and a separate age-depth equation for the interval $65-225 \mathrm{~cm}$. This interval can be dated by fitting a line to the oldest $210 \mathrm{~Pb}$ age of 88 years at $65 \mathrm{~cm}$ and lowermost three radiocarbon dates. A ges in this latter model are 20-60 years older in the interval from $\sim 65$ to $190 \mathrm{~cm}$; thus it is important to keep in mind the age uncertainty. 


\section{RESULTS}

\section{O stracodes}

Core SEI297-FB-1 can be divided into three informal faunal zones based on changes in the relative frequencies of various environmentally sensitive ostracode taxa (fig. 4, table 2). The lowermost zone (zone 1) from $226-175 \mathrm{~cm}$ is dominated by the species of the genera $\mathrm{N}$ eonesidea and Paranesidea (lumped into the family Bairdiidae), Radimella and $\mathrm{H}$ ermanites (plotted together), $\mathrm{N}$ eocaudites and A ctinocythereis. These species typically live exclusively in waters of normal marine salinity on carbonate platforms in tropical and transitional subtropical regions. The age of this zone is estimated to be about 1450-1600 AD (400-550 yr BP).

The second zone (zone 2) occurs from 175 to $65 \mathrm{~cm}$ and is characterized by common Loxoconcha matagordensis (20-50\%) and X estoleberis spp. (20-40\%). Loxochoncha. matagordensis is an epiphytal species that lives along Atlantic and Gulf of Mexico coasts of North America on Thalassia, Z ostera (in mid-latitudes), and other marine seagrasses (Cronin and others, in press). Many species of $X$ estoleberis inhabit various types of marine algae in tropical and subtropical regions. In addition to their preference for sub-aquatic vegetation habitats, L. matagordensis and several species of $X$ estol eberis thrive in environments of reduced salinity, such as those found in coastal bays and lagoons. Therefore, the dominance of epiphytal euryhaline ostracode species in this zone suggests a period of both abundant seagrass and marine al gal habitats and also fluctuating salinity between about 20-35 ppt. The age of this zone is estimated to be about 1600-1850 AD (150-400 yr BP).

The third zone (zone 3) occurs in the interval from $65 \mathrm{~cm}$ to the top of the core and represents the period from 1850 to 1997. The ostracode assemblages consist mostly of carbonate platform species as were found in zone 1, and in addition, $10 \%$ or more of $\mathrm{M}$ alzella floridana. This latter species is a dominant form 
Figure 4. Ostracodes (in percent abundance) from Featherbed Bank Core, Biscayne Bay.
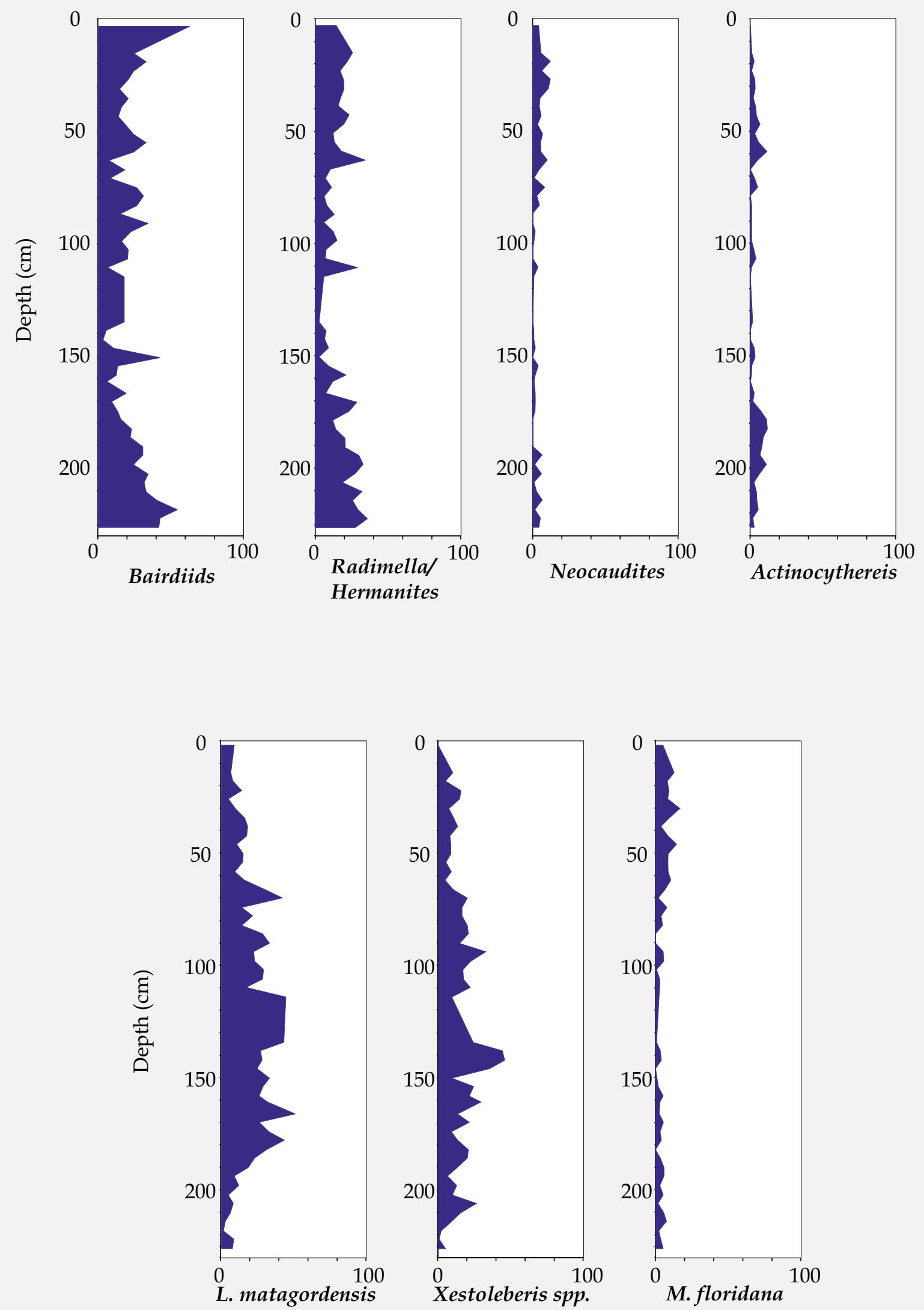
in Florida Bay in regions that experience periodic hypersalinity. While its presence in Biscayne Bay during the last 150 years does not necessarily indicate hypersal inity, it does distinguish the assemblages of zone 3 from zone 1. Epiphytal species al so decrease significantly in the transition from zone 2 to zone 1.

\section{Molluscs}

Examination of the molluscan fauna in Core SEI 297-FB-1 resulted in the identification of 96 faunal groups. The dominant species include Bittiolum varium, Turbo castaneus, Eulithidium sp., Tegula fasciata, and V ermicularia spirata. The assemblages and the molluscan faunal richness and abundance throughout the core indicate open-bay to open-marine conditions. On the basis of the molluscan assemblages, the salinity never dropped below $20 \mathrm{ppt}$, and was probably greater than 25 ppt throughout the time of deposition at this site. There are no molluscan indicators of proximity to land at this site.

The lower portion of Core SEI297-FB-1, from 226 to $175 \mathrm{~cm}$ (zone 1), is characterized by the presence of infaunal pelecypods (Pitar sp., Parastarte triquetra, Gouldia sp., N ucula proxima), perhaps indicating softer sediment available for habitation. Epiphytic and epibenthic gastropods (Eulithidium sp., Cerithium spp., Tegula fasciata, V ermicularia spirata, M odulus modulus, and Turbo castaneus) are also present in the lower portion of the core (fig. 5, table 3).

Bittiolum varium, a dominant epiphytic species in the upper portion of the core, is nearly absent from zone 1 . A verage molluscan faunal richness 1 is 13 and average abundance is 23 in the lower portion of the core; these numbers do not fluctuate significantly in this portion of the core, suggesting a relatively stable ecosystem. The molluscan fauna indicate near normal marine sal inities for zone 1. Samples between 190 and $175 \mathrm{~cm}$ mark a transition from zone 1 to zone 2 .

\footnotetext{
${ }^{1}$ Faunal richness is a measure of the number of faunal groups present in a given sample. In some cases, the fauna are grouped into genera and occasionally broader categories (e.g. marginellids), so this is not "species" richness in the usual sense.
} 
Figure 5. Molluscs (in percent abundance) from Featherbed Bank Core, Biscayne Bay.
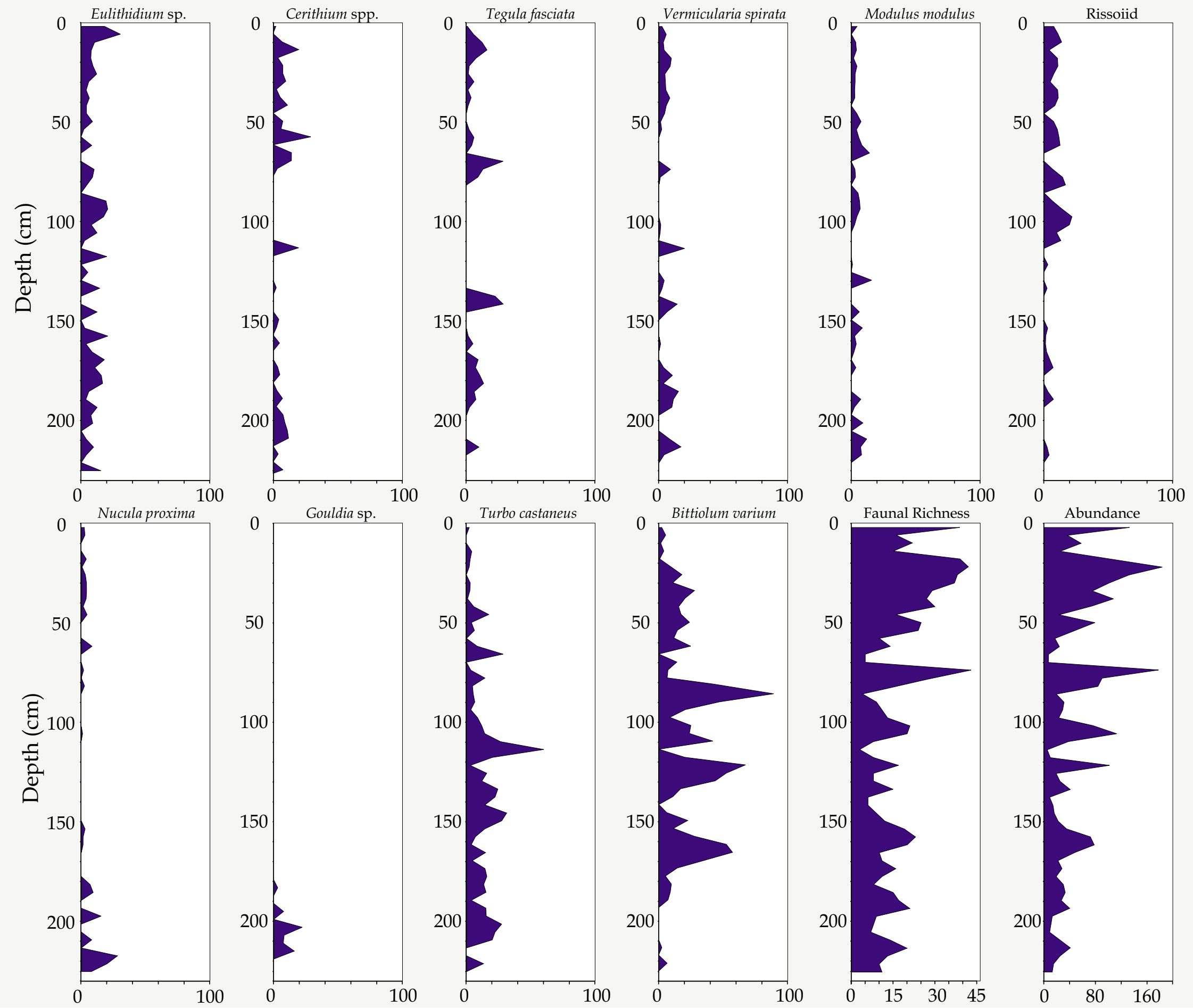
The middle portion of the core, from $175-65 \mathrm{~cm}$ (zone 2), is dominated by the presence of Bittiolum varium; T urbo castaneus also is relatively abundant. Burrowing pelecypods are sparse in zone 2 and almost completely absent from $118-90 \mathrm{~cm}$, indicating the substrate may not have been suitable for habitation; this corresponds to an increase in organisms that live on hard substrates and/ or sub-aquatic vegetation. Average molluscan faunal richness in the middle portion of the core is 12, nearly the same as the lower portion, but abundance is highly variable compared to zone 1, averaging 39 individuals per sample. The molluscan fauna indicate the salinity in this portion of the core may have been reduced periodically (perhaps as low as $25 \mathrm{ppt}$ ) compared to zone 1; the presence of several species that require near normal marine salinities, however, discounts sustained periods of lower salinities. Samples between 90 and $65 \mathrm{~cm}$ mark a transition from zone 2 to zone 3 , sharing characteristics of both.

The upper portion of the core, from 65 to $0 \mathrm{~cm}$ (zone 3), is marked by a decline in the dominance of Bittiol um varium, and a dramatic increase in faunal richness to an average of 25 faunal groups per sample. Cerithium spp., Tegula fasciata, V ermicularia spirata, M odulus modulus, rissoinids, N ucula proxima, and Transenella sp. increase in numbers in the upper portion of the core. This portion of the core contains a distinct assemblage very similar to the western margin of Florida Bay. The assemblage is dominated by Turbo castaneus and Tegula fasciata with minor percentages of Brachidontes exustus, Pteria longisquamosa, Cerithium muscarum, Chione cancellata, and other species more common in eastern and central Florida Bay. The assemblage requires relatively stable, near-normal marine sal inities (Brewster-Wingard, Stone, and Holmes, in press). This evidence is supported by the presence of $\mathrm{H}$ alimeda, in the upper $80 \mathrm{~cm}$ of the core. Halimeda is a green algae common in normal marine conditions and found along the western margin of Florida Bay. Faunal abundance, which fluctuates dramatically in zone 3, ranging from 7 to 184 individuals per sample (averaging 74), distinguishes this assemblage from the molluscan assemblage in zone 1. 


\section{Foraminifers}

The foraminiferal analyses resulted in the identification of 41 species (table 4). The assemblages are dominated by miliolid forms, with calcareous hyal ine taxa common, and agglutinated taxa rare. The dominant species include Q uinquel oculina bosciana, Q . poeyana, Q . polygona, Q . seminula, Q . tenagos, Triloculina trigonula, M iliolinella circularis, EIphidium delicatulum, A rchaias angulatus, A rticulina mucronata, Rosalina floridana, and R. globularis. Other subsidiary species include the rotaliids A mmonia parkinsoniana tepida, A . parkinsoniana typica, and Florilus auriculus, and the textulariid Clavulina tricarinata (fig. 6).

Ishman (in press) recognized four benthic foraminiferal assemblages from surficial sediment samples collected in Biscayne Bay. The foraminiferal faunas from Core SE1297-FB-1 represent a mixture of the open-bay grass assemblage and the open-bay coarse sediment assemblage, indicating open-bay normal circulation throughout the deposition of this core.

From the core bottom to $175 \mathrm{~cm}$ (zone 1), an open-bay seagrass form Elphidium delicatulum dominates to the near exclusion of other members of the seagrass assemblage. Within this interval, a variety of open-bay coarse sediment forms of the genera Q uinqueloculina (Q . tenagos, Q . poeyana, Q. polygona) and Triloculina (T. triacarinata) and Rosalina (R. floridana) are present to common, suggesting a patchy seagrass-bare substrate environment.

From $175 \mathrm{~cm}$ to $65 \mathrm{~cm}$ (zone 2), Elphidium delicatulum still dominates, but other representatives of the seagrass assemblage, such as Q uinquel oculina bosciana, A rticulina mucronata, and M iliammina circularis, are present to common, and forms of the open-bay coarse sediment assemblage are still present but less abundant, indicating an environment with fairly healthy seagrass and sparse, patchy bare sediment. Samples between 100 and $65 \mathrm{~cm}$ mark a transition from zone 2 to zone 3 assemblages. Foraminifer assemblages, commonly found in minor relative percentages in sal inities that range as low as $30 \mathrm{ppt}$, are present 
Figure 6. Foraminifers (in percent abundance) from Featherbed Bank Core, Biscayne Bay.
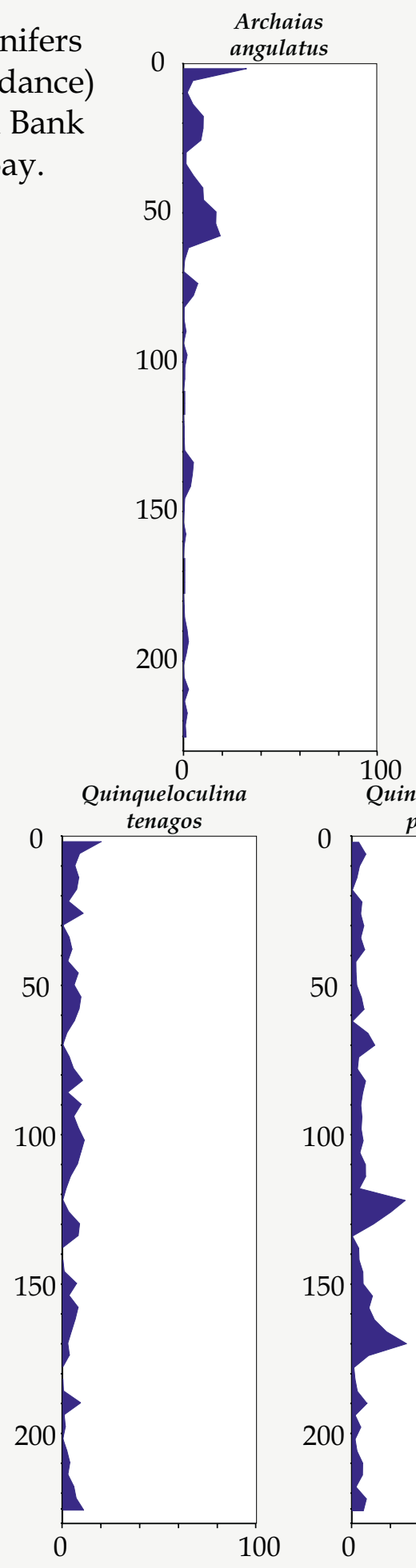
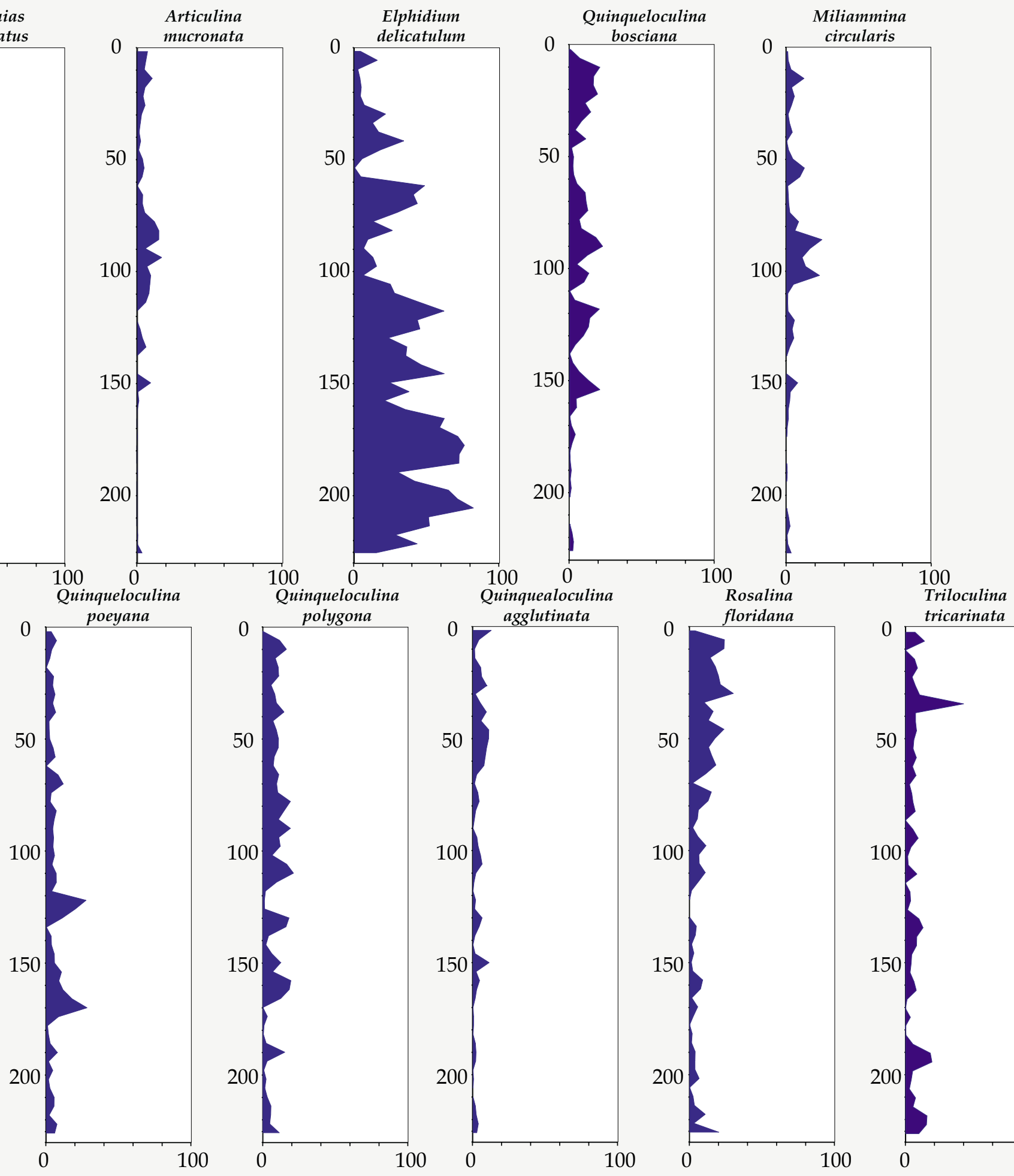

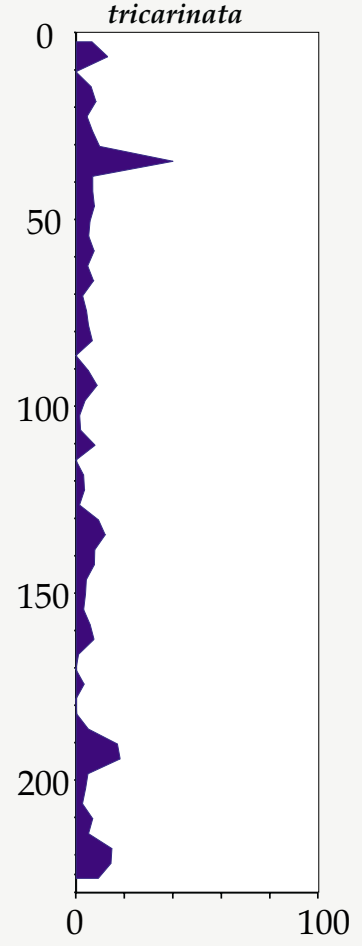


within zone 2 , suggesting that salinity conditions may have been slightly reduced when compared with zone 1.

From $65 \mathrm{~cm}$ to the top of the core (zone 3), the open-bay coarse sediment foraminiferal assemblage attains dominance. There is an increased abundance of most members of the open-bay coarse sediment assemblage in zone 3 and Elphidium delicatulum is greatly reduced, only dominating in a few horizons; other forms of the seagrass indicators remain unchanged or show only a modest increase in abundance. This suggests a bare sediment environment with patchy seagrass gradually replacing the lush sub-aquatic vegetation from zone 2 in the upper part of the core. 


\section{DISCUSSION}

\section{Regional Model for Featherbed Bank}

The results of faunal analyses in this study have produced a model for Core SEI 297-FB-1 that divides the fossil assemblages into three informal faunal zones. Although preliminary in nature, the results suggest distinct environmental changes have characterized deposition at Featherbed Bank over the past 5 centuries (fig. 7).

The data from zone 1 (base to $175 \mathrm{~cm}$ ) indicate that during the $15^{\text {th }}$ and $16^{\text {th }}$ centuries Featherbed Bank had an open-marine carbonate environment. The substrate during this period was patchy sub-aquatic vegetation, with a strong infaunal presence. Key biotic indicators suggest that salinity within zone 1 remained relatively stable and near to normal marine throughout deposition.

Zone $2(175-65 \mathrm{~cm})$ deposited during the $17^{\text {th }}, 18^{\text {th }}$, and $19^{\text {th }}$ centuries, had a thriving bottom community living on seagrasses (probably abundant Thalassia) and marine algae. The presence of some taxa capable of tolerating lowered salinities in zone 2 indicate that this period may have experienced a wetter regional climate and/ or experienced greater influx of freshwater. However, relatively high abundances of biotic indicators, which require near normal marine sal inities within zone 2 , limits the potential that salinity conditions were lowered for a sustained period.

Zone $3(65-0 \mathrm{~cm})$ represents the last 150 years of Biscayne Bay history when open-marine species re-colonized and sub-aquatic vegetation decreased. For the first time, the ostracode $\mathrm{M}$ alzella floridana became common, indicating possible episodes of hypersalinity and a molluscan assemblage similar to the western margin of Florida Bay appeared. The dramatic change in molluscan faunal richness and abundance in the upper portion of the core is striking (fig. 7). It is possible that this is a taphonomic effect, but preservation quality of 
Figure 7. SEI297-FB-1 Key Down-core Indicators Plotted Against Time.
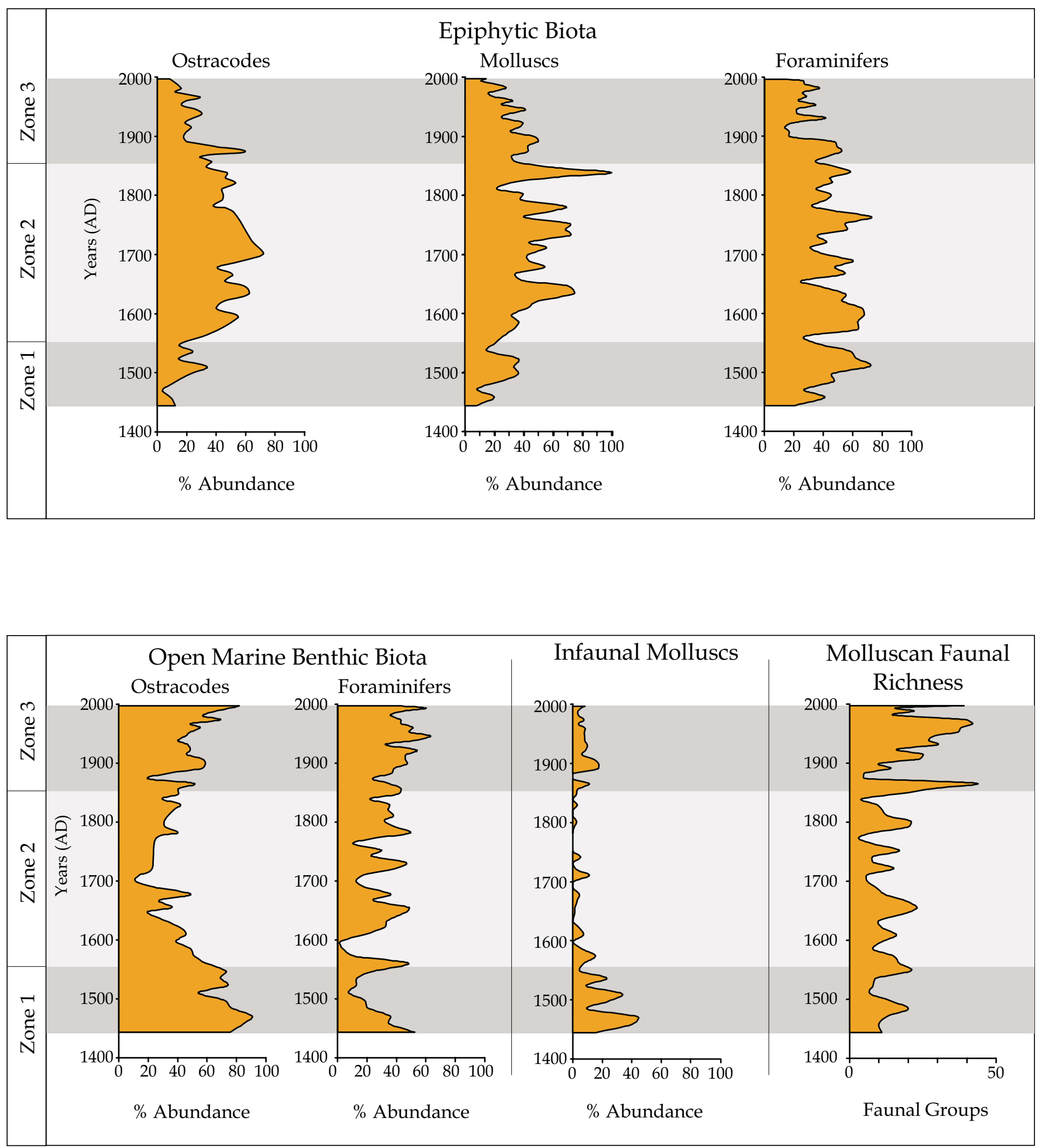
molluscan fossil shells is generally the same throughout the core; for example, the lowest portion of the core contains very delicate specimens that are well preserved, and color patterns are still present. These observations indicate that the variation in faunal richness and abundance is real and not a taphonomic bias. The increase in molluscs may be related to the variability in substrate heterogeneity, to fluctuating salinities, or to some other factor such as productivity or increased nutrients.

\section{Bank Migration Model}

While distinct, the changes within core SEI297-FB-1 are dominated by assemblages that primarily indicate different substrates. Variation in the fossil biotic communities throughout the core indicate limited variation in salinity conditions, possibly ranging as low as $25 \mathrm{ppt}$ in zone 2; biological proxy indicators suggest that salinity conditions at Featherbed Bank remained stable and near normal marine throughout most of the deposition of the core.

The transition from infaunal and coarse-sediment dwelling biota (zone 1) to prominent epiphytic communities (zone 2) to variable substrates and potentially increased salinity fluctuations (zone 3 ) is consistent with a model for bank migration; the transition from zone 1 to zone 3 may be explained by gradual shallowing of the environment of deposition, with lush sub-aquatic vegetation (zone 2) representing the side of a migrating bank. Without further investigation, it would be difficult to determine if the changes seen in core SEI297-FB-1 are the result of local bank migration or if they represent regional changes within central Biscayne Bay over the past 5 centuries.

\section{Suggestions for Future W ork}

To date, short cores, like the core analyzed in this paper, have been taken from six different regions of Biscayne Bay for the USGS 'Ecosystem History of Biscayne Bay and the Southeast Coast' project (Ishman, 1997). Faunal analyses of 
the cores from Manatee Bay and Featherbed Bank have now been completed, and modern anal og data exist that al low a more accurate interpretation of historical trends in faunal assemblages. Results from this study suggest that environmental trends within Biscayne Bay show three distinct faunal zones, representing a shift in environmental conditions at Featherbed Bank. To understand whether these changes are a result of bay-wide changes or simply a local phenomenon produced by the migration of Featherbed Bank, additional cores must be analyzed from other regions within Biscayne Bay. 


\section{CONCLUSIONS}

Ostracode, molluscan, and foraminiferal biological proxy indicators demonstrate that Featherbed Bank experienced three distinct environments. From approximately 1440-1550AD (zone 1), biotic assemblages show that openbay, relatively stable marine, carbonate deposition dominated the environment. Patchy to limited sub-aquatic vegetation was present. Between 1550 and 1850 AD (zone 2), this environment gave way to open-bay conditions with lush subaquatic vegetation with only limited bare substrate. Salinity conditions may have reached as low as $25 \mathrm{ppt}$ within this zone, but probably not for sustained periods. Between 1850 and 1997 AD (zone 3), biotic assemblages, representing open-bay deposition with patchy sub-aquatic vegetation and increasing bare substrate, recolonize the environment. However, assemblages after 1850 display distinct differences from those in zone 1 , suggesting modest changes in salinity regimes and substrate. There is no evidence for proximity to land or sustained periods of lowered salinity throughout core SEI297-FB-1. Although distinct, the changes in biotic assemblages down-core are dominantly substrate-controlled and may represent either bay-wide or local variations in the biological communities at Featherbed Bank. 


\section{REFERENCES}

A bbott, R. T., 1974, American Seashells, $2^{\text {nd }}$ Edition. Van N ostrand Reinhold Co. New York. 663 p.

A ndrews, J., 1971, Shells and shores of Texas: Austin, TX, University of Texas, $365 \mathrm{p}$.

Bock, W. D., Lynts, G.W., Smith, S., Wright, R., Hay, W.W., and Jones, J.I., 1971, A symposium of Recent south Florida Foraminfera: Miami Geological Society Memoir 1, Miami Geological Society Publication, Miami, Florida.

Brewster-Wingard, G.L., Ishman, S.E., Edwards, L.E., and Willard, D.A., 1996, Preliminary report on the distribution of modern fauna and flora at selected sites in north-central and north-eastern Florida Bay: U.S. Geological Survey Open-File Report 96-0732, 36 p.

Brewster-Wingard, G.L., and Ishman, S.E., 1999, Historical trends in salinity and substrate in Central Florida Bay: A paleoecological reconstruction using modern analogue data: Estuaries v. 22, p. 369-383.

Brewster-Wingard, G.L., Stone, J.R., and Holmes, C.W., in press, Molluscan faunal distribution in Florida Bay, past and present: An integration of down-core and modern data. in Wardlaw, B.R., (ed.), Ecosystem history of South Florida: Bulletins of American Paleontology.

Cronin, T.M., Holmes, C.W., Wingard, G.L., Ishman, S.E., Dowsett, H., Keyser, D., and Waibel, N ., in press, Historical trends in epiphytal ostracodes from Florida Bay: implications for seagrass and macro-benthic al gal variability. in Wardlaw, B.R., (ed.), Ecosystem history of South Florida: Bulletins of A merican Paleontology.

Garbett, E.C., and M addocks, R.F., 1978, Zoogeography of Holocene cytheracean ostracodes in the bays of Texas: Journal of Paleontology v. 53, p. 841-919.

Ishman, S.E., and D'A mbrosio, J., 1997, Modern benthic foraminifer distributions in Biscayne Bay: A nalogues for historical reconstructions: U.S. Geological Survey Open-File Report 97-0034, 20 p.

Ishman, S.E, 1997, Ecosystem history of south Florida: Biscayne Bay sediment core descriptions: U.S. Geological Survey Open-File Report 97-0437, 15 p. 
Ishman, S.E., Cronin, T.M., Brewster-Wingard, G.L., Willard, D.A., and Verardo, D.J., 1998, A record of ecosystem change, Manatee Bay, Barnes Sound, Florida: Journal of Coastal Research, Special Issue, v. 26, p. 125-138.

Ishman, S.E., in press, Ecological controls on benthic foraminifer distributions in Biscayne Bay, Florida. in Wardlaw, B.R., (ed.), Ecosystem history of South Florida: Bulletins of A merican Paleontology.

Keyser, D., 1975, Ostracoden aus den Mangrovegebieten von Sudwest-Florida: A bhandlungen des $\mathrm{N}$ aturwissenschaftlichen Vereins in Hamburg: NF 18/ 19, p. 255-290.

Keyser, D., 1976, Zur kenntnis der brackigen mangrovebewachsenen Weichboden Sudwest-Floridas unter besonderer Berucksichtigung ihrer Ostracodenfauna: Ph. D. Dissertation, Hamburg University, 142 p.

Keyser, D., 1977, Brackwasser-Cytheracea aus Sud-Florida: A bhandlungen des Naturwissenschaftlichen Vereins in Hamburg: NF 20, p. 43-85.

Lidz, B.H., and Rose, P.R., 1989, Diagnostic foraminiferal assemblages of Florida Bay, and adjacent shallow water: a comparison: Bulletin of Marine Science v. 44, p. 399-418.

Loeblich, A.R., and Tappan, H., 1988, Foraminiferal genera and their classification, v. 1, 2: New York, Van N ostrand Reinhold Company, Inc., $970 \mathrm{p}$.

Lyons, W.G., 1996, An assessment of mollusks as indicators of environmental change in Florida Bay: in Programs and A bstracts, Florida Bay Science Conference, 1996, Gainesville, Florida, p. 52-54.

Lyons, W.G., 1999, Responses of benthic fauna to salinity shifts in Florida Bay: evidence from a more robust sample of the molluscan community: in Program and Abstracts, Florida Bay and A djacent Marine Systems Science Conference, November 1999, Key Largo, Florida, p 47-50.

Perry, L. M. and Schwengel, J.S., 1955, Marine shells of the western coast of Florida: Ithaca, NY, Paleontological Research Institution, 318 p.

Robbins, J.A., Holmes, C.W., Halley, R.B., Bothner, M., Shinn, E.A., Graney, J., Keller, G., tenBrinck, M., and Rudnick, D, in press, Time constraints characterizing predeposition integration of ${ }^{137} \mathrm{Ce}$ and $\mathrm{Pb}$ fluxes to sediments in Florida Bay: Geochemica et Cosmochemica. 
Stuvier, M., and Reimer, P.J., 1993, Extended (super 14) C data based and revised CALIB 3.0 (super 14) C age calibration program: Radiocarbon, v. 35, p. 215-230.

Teeter, J.W., 1975, Distribution of Holocene marine Ostracoda from Belize, in, Wantland, K.F., and Pusey, C., III, eds., Belize shelf-carbonate sediments, clastic sediments, and ecology: A merican Association of Petroleum Geologists Studies in Geology N o. 2, p. 400-498.

Turgeon, D.D., Quinn, J.F., Jr., Bogan, A.E., Coan, E.V., Hochberg, F.G., Lyons, W.G., Mikkelsen, P.M., N eves, R.J., Roper, C.F.E., Rosenberg, G., Roth, B., Scheltema, A., Thompson, F.G., Vecchione, M., and Williams, J.D., 1998, Common and scientific names of aquatic invertebrates from the United States and Canada: mollusks, 2nd Edition. American Fisheries Society, Special Publication 26, Bethesda, Maryland, $526 \mathrm{p}$.

Turney, W.J. and Perkins, B.F., 1972, M olluscan Distribution in Florida Bay: Sedimenta III, University of Miami, Miami, Florida, 37p.

Wanless, H.R., Cottrell, D.J., Tagett, M.G., Tedesco, L.P., and Warzeski, E.R., Jr., 1995, Origin and growth of carbonate banks in south Florida. In M onty, C.V., Boscence, D.W.J., Bridges, P.H., and Pratt, B.R., (eds.), Carbonate Mud-M ounds. Special Publication of the International Association of Sedimentologists, v. 23, p. 439-473.

Warmke, G.L., and A bbott, R.T., 1961, Carribean Seashells: Narberth, PA, Livingston Publishing Co. $348 \mathrm{p}$. 
Table 1. A ge model for core SEI297-FB-1 samples

\begin{tabular}{|c|c|c|c|c|c|}
\hline Depth (cm) & Method & Age\# & 2 Sigma Range & Uncorrected C-14 Age & $+1-$ \\
\hline $0-2$ & lead-210 & 1.66 & & & \\
\hline $2-4$ & lead-210 & 5.04 & & & \\
\hline $4-6$ & lead-210 & 8.49 & & & \\
\hline 6-8 & lead-210 & 12.00 & & & \\
\hline $8-10$ & lead-210 & 15.57 & & & \\
\hline $10-12$ & lead-210 & 19.20 & & & \\
\hline $12-14$ & lead-210 & 22.86 & & & \\
\hline $14-16$ & lead-210 & 26.55 & & & \\
\hline $16-18$ & lead-210 & 30.24 & & & \\
\hline $18-20$ & lead-210 & 33.90 & & & \\
\hline $20-22$ & lead-210 & 37.52 & & & \\
\hline $22-24$ & lead-210 & 41.06 & & & \\
\hline $24-26$ & lead-210 & 44.47 & & & \\
\hline $26-28$ & lead-210 & 47.71 & & & \\
\hline $28-30$ & lead-210 & 50.75 & & & \\
\hline $30-32$ & lead-210 & 53.54 & & & \\
\hline $32-34$ & lead-210 & 56.05 & & & \\
\hline 34-36 & lead-210 & 58.25 & & & \\
\hline $36-38$ & lead-210 & 60.15 & & & \\
\hline $38-40$ & lead-210 & 61.74 & & & \\
\hline $40-42$ & lead-210 & 63.05 & & & \\
\hline $42-44$ & lead-210 & 64.13 & & & \\
\hline $44-46$ & lead-210 & 65.05 & & & \\
\hline $46-48$ & lead-210 & 65.85 & & & \\
\hline $48-50$ & lead-210 & 66.64 & & & \\
\hline $50-52$ & lead-210 & 67.49 & & & \\
\hline $52-54$ & lead-210 & 68.51 & & & \\
\hline $54-56$ & lead-210 & 69.81 & & & \\
\hline $56-58$ & lead-210 & 71.52 & & & \\
\hline $58-60$ & lead-210 & 73.83 & & & \\
\hline $60-62$ & lead-210 & 76.99 & & & \\
\hline $62-64$ & lead-210 & 81.40 & & & \\
\hline $64-68$ & lead-210*k & 87.77 & & & \\
\hline $60-62$ & radiocarbon* & 295 & $350-0$ yr BP & 570 & 55 \\
\hline $100-102$ & radiocarbon** & 286 & $342-0$ yr BP & 555 & 55 \\
\hline $140-142$ & radiocarbon** & 359 & 503-303 yr BP & 695 & 55 \\
\hline $220-222$ & radiocarbon** & 505 & 567-363yr BP & 810 & 55 \\
\hline
\end{tabular}

*not used in age-depth model

* used to derive age model for interval $65-225 \mathrm{~cm}$ age $=0.004$ (depth*depth) $+(1.581 *$ depth $)-5.312$

\#Radiocarbon ages are calibrated using program in Stuvier and Reimer (1993) 


\begin{tabular}{|c|c|c|c|c|c|c|c|c|c|c|c|c|c|}
\hline Depth (cm) & Years (B.P.) & Age Model Year & 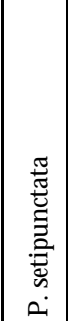 & 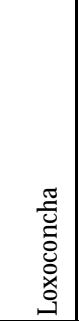 & $\begin{array}{l}\frac{\sigma}{\bar{N}} \\
\frac{N}{N} \\
\sum\end{array}$ & $\begin{array}{l}\frac{n}{8} \\
\frac{8}{0} \\
\frac{0}{0} \\
\times\end{array}$ & $\begin{array}{l}\frac{n}{0} \\
: \bar{D} \\
. \bar{D} \\
\infty\end{array}$ & $\begin{array}{l}\frac{0}{0} \\
\frac{0}{0} \\
\frac{1}{2} \\
0\end{array}$ & 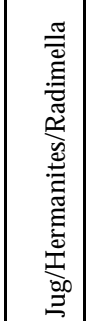 & 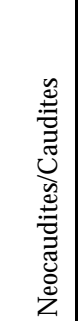 & 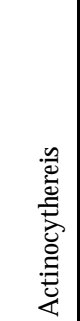 & $\begin{array}{l}\bar{\Phi} \\
\frac{ \pm}{ \pm}\end{array}$ & 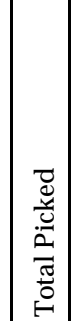 \\
\hline $0-2$ & 0.00 & 1997 & 0.00 & 8.65 & 4.81 & 0.00 & 63.46 & 0.00 & 14.42 & 3.85 & 0.00 & 4.81 & 1104 \\
\hline $12-14$ & 15.92 & 1981 & 0.00 & 6.36 & \begin{tabular}{|l|}
11.82 \\
\end{tabular} & 10.00 & 25.45 & 0.00 & 25.45 & 5.45 & 0.91 & 14.55 & \begin{tabular}{|l|l|}
5110 \\
\end{tabular} \\
\hline $16-18$ & 22.72 & 1974 & 0.00 & 7.50 & 7.50 & 5.00 & 33.33 & \begin{tabular}{|l|}
0.00 \\
\end{tabular} & 21.67 & 11.67 & 2.50 & 10.83 & \begin{tabular}{|l|l|}
3 & 120 \\
\end{tabular} \\
\hline $20-22$ & 29.65 & 1967 & 0.85 & 13.68 & 8.55 & 15.38 & 24.79 & 0.85 & 17.09 & 5.98 & 0.85 & 11.97 & \begin{tabular}{l|l}
7117 \\
\end{tabular} \\
\hline $24-26$ & 36.71 & 1960 & 0.00 & 4.85 & 7.77 & 14.56 & 21.36 & \begin{tabular}{|l|}
0.97 \\
\end{tabular} & 19.42 & 11.65 & 2.91 & 16.50 & \begin{tabular}{|l|l|} 
& 103 \\
\end{tabular} \\
\hline $28-30$ & 43.90 & 1953 & 0.00 & 9.28 & 15.46 & 7.22 & 15.46 & \begin{tabular}{|l|}
4.12 \\
\end{tabular} & 19.59 & 10.31 & 3.09 & 15.46 & \begin{tabular}{|l|l|}
5 & 97 \\
\end{tabular} \\
\hline $32-34$ & 51.22 & 1946 & 0.00 & 15.38 & 8.65 & 10.58 & 21.15 & 3.85 & 17.31 & 4.81 & 1.92 & 16.35 & \begin{tabular}{|l|l|} 
& 104 \\
\end{tabular} \\
\hline $36-38$ & 58.66 & 1938 & 0.00 & 17.39 & 3.48 & \begin{tabular}{|l|}
13.04 \\
\end{tabular} & \begin{tabular}{|l|}
16.52 \\
\end{tabular} & 4.35 & 15.65 & 4.35 & 3.48 & 21.74 & \begin{tabular}{l|l|}
4 & 115 \\
\end{tabular} \\
\hline $40-42$ & 66.23 & 1931 & 0.79 & 16.67 & 7.94 & 7.94 & 14.29 & \begin{tabular}{|l|}
4.76 \\
\end{tabular} & 23.02 & 5.56 & 3.97 & 15.08 & \begin{tabular}{|l|l|} 
& 126 \\
\end{tabular} \\
\hline \begin{tabular}{|l|}
$44-46$ \\
\end{tabular} & 73.93 & 1923 & 1.56 & 10.16 & 13.28 & 8.59 & \begin{tabular}{|l|}
19.53 \\
\end{tabular} & \begin{tabular}{|l|}
2.34 \\
\end{tabular} & 19.53 & 3.13 & 6.25 & 15.63 & \begin{tabular}{|l|l|}
328 \\
\end{tabular} \\
\hline $48-50$ & 81.76 & 1915 & \begin{tabular}{|l|}
0.72 \\
\end{tabular} & 14.49 & 7.97 & 8.70 & 24.64 & \begin{tabular}{|l|}
1.45 \\
\end{tabular} & 12.32 & 6.52 & 2.90 & 20.29 & \begin{tabular}{|l|l}
9 & 138 \\
\end{tabular} \\
\hline $52-54$ & 89.72 & 1907 & 0.00 & 14.04 & 7.89 & 5.26 & 33.33 & \begin{tabular}{|l|}
0.88 \\
\end{tabular} & 13.16 & 5.26 & 5.26 & 14.91 & \begin{tabular}{|l|l|}
1114 \\
\end{tabular} \\
\hline $56-58$ & 97.80 & 1899 & 0.89 & 8.93 & 8.04 & 8.93 & 25.00 & 2.68 & 17.86 & 5.36 & 10.71 & 11.61 & \begin{tabular}{|l|l|}
112 \\
\end{tabular} \\
\hline $60-62$ & 106.01 & 1891 & \begin{tabular}{|l|}
0.81 \\
\end{tabular} & 15.32 & 9.68 & 4.84 & 8.06 & \begin{tabular}{|l|}
0.81 \\
\end{tabular} & 33.87 & 9.68 & 4.84 & 12.10 & \begin{tabular}{|l|l|} 
& 124 \\
\end{tabular} \\
\hline 64-66 & 114.35 & 1883 & 0.87 & 28.70 & 6.09 & 10.43 & \begin{tabular}{|l|}
19.13 \\
\end{tabular} & \begin{tabular}{|l|}
2.61 \\
\end{tabular} & 10.43 & 4.35 & 0.00 & 17.39 & \begin{tabular}{|l|l|}
9 & 115 \\
\end{tabular} \\
\hline 68-70 & 122.82 & 1874 & 5.36 & 40.18 & 1.79 & \begin{tabular}{|l|}
19.64 \\
\end{tabular} & 8.93 & \begin{tabular}{|l|}
0.89 \\
\end{tabular} & 7.14 & 0.89 & 2.68 & 12.50 & 112 \\
\hline 72-74 & 1.42 & 1866 & \begin{tabular}{|l|}
1.60 \\
\end{tabular} & 13.60 & 7.20 & 16.00 & 27.20 & \begin{tabular}{|l|}
0.00 \\
\end{tabular} & 11.20 & 8.00 & 4.80 & 10.40 & 125 \\
\hline 76-78 & 140.14 & 1857 & \begin{tabular}{|l|}
0.00 \\
\end{tabular} & 20.72 & 3.60 & \begin{tabular}{|l|}
16.22 \\
\end{tabular} & 31.53 & \begin{tabular}{|l|}
0.90 \\
\end{tabular} & 6.31 & 2.70 & 0.00 & 18.02 & 2111 \\
\hline $80-82$ & 148.99 & 1848 & 0.00 & 13.51 & 4.50 & 19.82 & 27.03 & 0.00 & 8.11 & 4.50 & 0.90 & 21.62 & \begin{tabular}{|l|}
111 \\
\end{tabular} \\
\hline $84-86$ & 157.97 & 1839 & 0.00 & 27.05 & 0.00 & 20.49 & 15.57 & 2.46 & 13.11 & 0.00 & 0.82 & 20.49 & \begin{tabular}{|l|l}
9 & 122 \\
\end{tabular} \\
\hline $88-90$ & 167.08 & 1830 & 0.00 & 31.62 & 0.00 & 14.53 & 35.04 & 0.00 & 5.98 & 0.00 & 0.85 & 11.97 & \begin{tabular}{l|l}
7117 \\
\end{tabular} \\
\hline $92-94$ & 176.32 & 1821 & 0.82 & 21.31 & 4.92 & 31.97 & 22.95 & 0.00 & 12.30 & 1.64 & 0.82 & 3.28 & \begin{tabular}{|l|l}
3 & 122
\end{tabular} \\
\hline $96-98$ & .68 & 1811 & 0.00 & 21.93 & 5.26 & 21.93 & 16.67 & 0.88 & 14.91 & 0.88 & 0.88 & 16.67 & \begin{tabular}{l|l}
7114 \\
\end{tabular} \\
\hline $100-102$ & 195.17 & 1802 & 0.00 & 28.03 & 0.76 & 16.67 & 21.21 & 0.00 & 7.58 & 0.00 & 2.27 & 23.48 & \begin{tabular}{|l|l|}
3 & 132 \\
\end{tabular} \\
\hline $104-106$ & 204.79 & 1792 & 0.00 & 27.03 & 2.70 & 17.12 & 20.72 & 1.80 & 7.21 & 0.00 & 3.60 & 19.82 & \begin{tabular}{l|l}
2111 \\
2111
\end{tabular} \\
\hline $108-110$ & 214.54 & 1782 & 1.74 & 16.52 & 2.61 & 21.74 & 6.96 & 0.00 & 28.70 & 3.48 & 0.87 & 17.39 & \begin{tabular}{|l|l|}
9115 \\
\end{tabular} \\
\hline $112-114$ & 224.42 & 1773 & 0.00 & 42.31 & 2.31 & 9.23 & 18.46 & 0.77 & 6.15 & 0.77 & 0.00 & 20.00 & 130 \\
\hline $132-134$ & 275.72 & 1721 & 0.74 & 41.18 & 0.74 & 23.53 & 18.38 & 0.00 & 2.94 & 0.00 & 1.47 & 11.03 & \begin{tabular}{|l|l}
3 & 136 \\
\end{tabular} \\
\hline $136-138$ & 286.36 & 1711 & 1.52 & 25.76 & 3.03 & 43.18 & 6.06 & 0.00 & 7.58 & 0.76 & 0.00 & 12.12 & \begin{tabular}{|l|l|}
2 & 132 \\
\end{tabular} \\
\hline $140-142$ & 297.13 & 1700 & 0.00 & 26.87 & 3.73 & 44.78 & 3.73 & 2.24 & 6.72 & 0.75 & 0.00 & 11.19 & \begin{tabular}{|l|l|}
9 & 134 \\
\end{tabular} \\
\hline $144-146$ & 308.03 & 1689 & 0.00 & 23.73 & 0.00 & 34.75 & \begin{tabular}{|l|}
11.02 \\
\end{tabular} & 1.69 & 9.32 & 1.69 & 2.54 & 15.25 & \begin{tabular}{|l|l|}
118 \\
\end{tabular} \\
\hline $148-150$ & 319.06 & 1678 & 1.02 & 31.63 & 1.02 & 9.18 & 42.86 & 0.00 & 3.06 & 0.00 & 3.06 & 8.16 & \begin{tabular}{|l|l|}
5 & 98 \\
\end{tabular} \\
\hline $152-154$ & 330.22 & 1667 & 0.00 & 27.52 & 1.83 & 23.85 & \begin{tabular}{|l|}
13.76 \\
\end{tabular} & \begin{tabular}{|l|}
0.92 \\
\end{tabular} & 9.17 & 3.67 & 0.92 & 18.35 & \begin{tabular}{|l|l|}
5109 \\
\end{tabular} \\
\hline 156-158 & 341.50 & 1655 & 0.81 & 25.00 & 4.84 & 20.97 & 12.90 & 0.00 & 20.97 & 1.61 & 0.81 & 12.10 & 124 \\
\hline \begin{tabular}{|l|}
$160-162$ \\
\end{tabular} & 350.05 & 1647 & 1.85 & 30.56 & 2.78 & 28.70 & 6.48 & \begin{tabular}{|l|}
0.00 \\
\end{tabular} & 12.04 & 0.93 & 0.00 & 16.67 & \begin{tabular}{|l|l|}
7 & 108 \\
\end{tabular} \\
\hline $164-166$ & 364.45 & 1633 & 1.65 & 48.76 & 2.48 & 13.22 & 19.83 & 0.00 & 7.44 & 1.65 & 2.48 & 2.48 & \begin{tabular}{|l|l}
121 \\
\end{tabular} \\
\hline $168-170$ & 376.12 & 1621 & 0.00 & 25.00 & 5.00 & 20.83 & 10.00 & 0.00 & 28.33 & 1.67 & 1.67 & 7.50 & 120 \\
\hline $172-174$ & 387.92 & 1609 & 0.00 & 31.34 & 2.99 & 8.96 & 14.18 & 0.00 & 23.13 & 1.49 & 6.72 & 11.19 & \begin{tabular}{|l|l|}
9 & 134 \\
\end{tabular} \\
\hline $176-178$ & 399.84 & 1597 & 0.00 & 41.51 & 3.77 & 13.21 & 16.04 & 0.00 & 12.26 & 0.00 & 10.38 & 2.83 & \begin{tabular}{|l|l|}
106 \\
\end{tabular} \\
\hline $180-182$ & 411.89 & 1585 & 0.00 & 29.69 & 0.00 & 20.31 & 23.44 & 0.00 & 14.06 & 0.00 & 10.94 & 1.56 & \begin{tabular}{|l|l}
5128 \\
5
\end{tabular} \\
\hline $184-186$ & 424.07 & 1573 & 0.00 & 21.97 & 3.03 & 19.70 & 22.73 & 0.00 & 20.45 & 0.00 & 8.33 & 3.79 & \begin{tabular}{|l|l}
932 \\
9
\end{tabular} \\
\hline 188-190 & 436.38 & 1561 & 0.00 & 17.69 & 5.44 & 12.93 & 31.29 & 0.00 & 20.41 & 0.00 & 7.48 & 4.76 & \begin{tabular}{|l|l}
5147 \\
5
\end{tabular} \\
\hline \begin{tabular}{|l|}
$192-194$ \\
\end{tabular} & 448.82 & 1548 & \begin{tabular}{|l|}
0.00 \\
\end{tabular} & 8.53 & 5.43 & 6.20 & 31.01 & \begin{tabular}{|l|}
0.00 \\
\end{tabular} & 29.46 & 6.20 & 6.20 & 6.98 & $\begin{array}{ll}129 \\
\end{array}$ \\
\hline 196-198 & 461.38 & 1536 & 0.00 & 11.72 & 2.76 & 12.41 & 24.83 & 0.00 & 32.41 & 1.38 & 10.34 & 4.14 & \begin{tabular}{|l|l|}
4 & 145 \\
\end{tabular} \\
\hline $200-202$ & 474.07 & 1523 & 0.00 & 4.85 & 4.85 & 9.71 & 34.95 & 0.00 & 27.18 & 5.83 & 5.83 & 6.80 & 103 \\
\hline $204-206$ & 486.89 & 1510 & 0.00 & 8.06 & 1.61 & 25.81 & 32.26 & 0.00 & 18.55 & 0.81 & 2.42 & 10.48 & \begin{tabular}{|l|l}
3 & 124
\end{tabular} \\
\hline $208-210$ & 499.84 & 1497 & 0.00 & 5.92 & 5.26 & 15.13 & 33.55 & 0.00 & 31.58 & 2.63 & 3.95 & 1.97 & \begin{tabular}{l|l}
752 \\
\end{tabular} \\
\hline $212-214$ & 512.92 & 1484 & 0.00 & 2.82 & 7.04 & 9.15 & 40.85 & 0.00 & 25.35 & 6.34 & 4.23 & 4.23 & \begin{tabular}{|l|l|}
142 \\
\end{tabular} \\
\hline $216-218$ & 526.12 & 1471 & 0.00 & 1.45 & 2.17 & 2.17 & 55.07 & 0.00 & 28.99 & 1.45 & 5.07 & 3.62 & 2138 \\
\hline $220-222$ & 539.45 & 1458 & 0.00 & 8.40 & 3.36 & 0.84 & 42.86 & 0.00 & 35.29 & 5.04 & 1.68 & 2.52 & \begin{tabular}{|l|l|}
119 \\
\end{tabular} \\
\hline $224-226$ & 552.91 & 1444 & 0.00 & 7.32 & 4.88 & 4.88 & 42.28 & 0.00 & 26.83 & 4.07 & 2.44 & 7.32 & \begin{tabular}{|l|l|}
123 \\
\end{tabular} \\
\hline
\end{tabular}




\begin{tabular}{|c|c|c|c|c|c|c|c|c|c|c|c|c|c|c|c|c|c|c|}
\hline Depth (cm) & Years (B.P.) & Age Model Year & $\begin{array}{l}\bar{y} \\
\frac{2}{0} \\
\frac{0}{8} \\
\frac{n}{4} \\
\frac{0}{0} \\
\frac{8}{4} \\
\frac{8}{4}\end{array}$ & 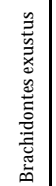 & 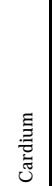 & 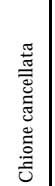 & 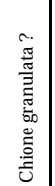 & 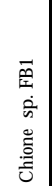 & $\begin{array}{l}\frac{n}{2} \\
\frac{0}{0} \\
\frac{0}{0} \\
\frac{0}{0} \\
0 \\
\frac{0}{0} \\
\frac{0}{8} \\
u\end{array}$ & $\begin{array}{l}\frac{\pi}{0} \\
\frac{\pi}{5} \\
\frac{0}{0} \\
\frac{0}{0} \\
\frac{\pi}{0} \\
\frac{7}{0} \\
\frac{\pi}{8} \\
ن\end{array}$ & 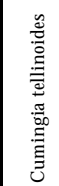 & 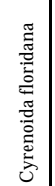 & 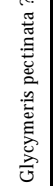 & $\begin{array}{l}\dot{\theta} \\
\dot{0} \\
\dot{0} \\
\overline{0} \\
\overline{0} \\
0\end{array}$ & 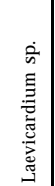 & 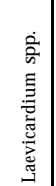 & 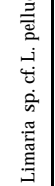 & 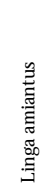 \\
\hline $0-2$ & 0.00 & 1997 & 0.00 & 0.00 & 0.00 & 0.75 & 0.00 & 3.76 & 0.00 & 5.26 & 0.75 & 0.00 & 3.76 & 0.00 & \begin{tabular}{|l|}
0.75 \\
\end{tabular} & \begin{tabular}{|l|}
0.00 \\
\end{tabular} & \begin{tabular}{|l|}
1.50 \\
\end{tabular} & 0.00 \\
\hline $4-6$ & 2.69 & 1994 & 2.78 & 0.00 & \begin{tabular}{l|l}
0.00 \\
\end{tabular} & 0.00 & 0.00 & 2.78 & 0.00 & 5.56 & 0.00 & 0.00 & 0.00 & 0.00 & \begin{tabular}{|l|}
0.00 \\
\end{tabular} & \begin{tabular}{|l|}
0.00 \\
\end{tabular} & 0.00 & 0.00 \\
\hline $8-10$ & 9.24 & 1988 & 1.72 & 0.00 & 0.00 & 0.00 & 0.00 & 0.00 & 6.90 & 5.17 & 0.00 & 0.00 & 0.00 & 0.00 & 0.00 & 0.00 & 0.00 & 1.72 \\
\hline \begin{tabular}{|l|}
$12-14$ \\
\end{tabular} & 15.92 & 1981 & 0.00 & 0.00 & 0.00 & 4.00 & 0.00 & 0.00 & 12.00 & 0.00 & 0.00 & 0.00 & 0.00 & 0.00 & 4.00 & \begin{tabular}{|l|}
0.00 \\
\end{tabular} & 4.00 & 0.00 \\
\hline $16-18$ & 22.72 & 1974 & 0.00 & 0.95 & 0.00 & 1.90 & 0.00 & 0.95 & 0.95 & 3.81 & 0.95 & 0.00 & 0.00 & 0.00 & 0.95 & 0.00 & 1.90 & 0.00 \\
\hline $20-22$ & 29.65 & 1967 & 0.00 & 0.54 & 0.00 & 0.00 & 0.00 & 0.54 & 2.17 & 3.26 & 1.09 & 0.00 & 0.00 & 0.00 & \begin{tabular}{|l|}
0.00 \\
\end{tabular} & \begin{tabular}{|l|}
0.00 \\
\end{tabular} & 0.54 & 0.00 \\
\hline $24-26$ & 36.71 & 1960 & 0.76 & 0.00 & 0.00 & 0.76 & 0.00 & 1.52 & 0.00 & 0.76 & 0.76 & 0.00 & 0.00 & 0.00 & 1.52 & 0.00 & \begin{tabular}{|l|}
1.52 \\
\end{tabular} & 0.00 \\
\hline $28-30$ & 43.90 & 1953 & 0.00 & 0.99 & 0.00 & 2.97 & 0.00 & 0.99 & 0.99 & 0.99 & 0.99 & 0.99 & 0.99 & 0.00 & 0.00 & 0.00 & 0.00 & 0.00 \\
\hline $32-34$ & 51.22 & 1946 & 0.00 & 1.33 & 0.00 & 2.67 & 0.00 & 0.00 & 4.00 & 1.33 & 0.00 & 0.00 & 0.00 & 0.00 & \begin{tabular}{|l|}
2.67 \\
\end{tabular} & 0.00 & \begin{tabular}{|l|}
2.67 \\
\end{tabular} & 0.00 \\
\hline 36-38 & 58.66 & 1938 & 0.00 & 1.85 & 0.00 & 0.00 & 0.00 & 2.78 & 0.00 & 3.70 & 1.85 & 0.00 & 0.00 & 0.00 & 0.00 & 0.00 & 0.93 & 0.00 \\
\hline \begin{tabular}{|l|}
$40-42$ \\
\end{tabular} & 66.23 & 1931 & 0.00 & 1.41 & 0.00 & 2.82 & 0.00 & 2.82 & 0.00 & 0.00 & 1.41 & 0.00 & 0.00 & 0.00 & 0.00 & 0.00 & 0.00 & 0.00 \\
\hline $44-46$ & 73.93 & 1923 & 0.00 & 4.35 & 0.00 & 0.00 & 0.00 & 4.35 & 0.00 & 0.00 & 0.00 & 0.00 & 0.00 & 0.00 & \begin{tabular}{|l|}
0.00 \\
\end{tabular} & \begin{tabular}{|l|}
0.00 \\
\end{tabular} & 0.00 & 0.00 \\
\hline \begin{tabular}{|l|}
$48-50$ \\
\end{tabular} & 81.76 & \begin{tabular}{|l|}
1915 \\
\end{tabular} & 0.00 & 0.00 & 0.00 & 2.53 & 0.00 & 1.27 & 0.00 & $\begin{array}{l}0.00 \\
\end{array}$ & 0.00 & 0.00 & 0.00 & 0.00 & \begin{tabular}{|l|}
0.00 \\
\end{tabular} & \begin{tabular}{|l|}
0.00 \\
\end{tabular} & \begin{tabular}{|l|}
0.00 \\
\end{tabular} & 0.00 \\
\hline 52-54 & 89.72 & 1907 & 0.00 & 0.00 & 0.00 & 6.25 & 0.00 & 8.33 & 0.00 & 2.08 & 0.00 & 0.00 & 0.00 & 0.00 & 0.00 & \begin{tabular}{|l|}
0.00 \\
\end{tabular} & 0.00 & 0.00 \\
\hline \begin{tabular}{|l|}
$56-58$ \\
\end{tabular} & \begin{tabular}{|c|}
97.80 \\
\end{tabular} & 1899 & 0.00 & 0.00 & 0.00 & 5.88 & 0.00 & 0.00 & 0.00 & 0.00 & 0.00 & 0.00 & 0.00 & 0.00 & \begin{tabular}{|l|}
0.00 \\
\end{tabular} & \begin{tabular}{|l|}
0.00 \\
\end{tabular} & \begin{tabular}{|l|}
0.00 \\
\end{tabular} & 0.00 \\
\hline 60-62 & 106.01 & 1891 & 0.00 & 4.17 & 0.00 & 4.17 & 0.00 & 0.00 & 0.00 & 0.00 & 0.00 & 0.00 & 0.00 & 0.00 & 0.00 & 0.00 & 0.00 & 0.00 \\
\hline \begin{tabular}{|l|}
$64-66$ \\
\end{tabular} & 114.35 & 1883 & 0.00 & 0.00 & 0.00 & 0.00 & 0.00 & $\begin{array}{l}0.00 \\
\end{array}$ & 0.00 & 0.00 & 0.00 & $\begin{array}{l}0.00 \\
\end{array}$ & $\begin{array}{l}0.00 \\
\end{array}$ & 0.00 & \begin{tabular}{|l|}
0.00 \\
\end{tabular} & \begin{tabular}{|l|}
0.00 \\
\end{tabular} & \begin{tabular}{|l|}
0.00 \\
\end{tabular} & 0.00 \\
\hline \begin{tabular}{|l|}
$68-70$ \\
\end{tabular} & \begin{tabular}{l|}
122.82 \\
\end{tabular} & 1874 & \begin{tabular}{l|}
0.00 \\
\end{tabular} & \begin{tabular}{l|}
0.00 \\
\end{tabular} & $\begin{array}{l}0.00 \\
\end{array}$ & $\begin{array}{l}0.00 \\
\end{array}$ & \begin{tabular}{l|}
0.00 \\
\end{tabular} & \begin{tabular}{l|}
0.00 \\
\end{tabular} & 0.00 & $\begin{array}{l}0.00 \\
\end{array}$ & 0.00 & \begin{tabular}{l|}
0.00 \\
\end{tabular} & $\begin{array}{l}0.00 \\
\end{array}$ & 0.00 & \begin{tabular}{|l|}
0.00 \\
\end{tabular} & \begin{tabular}{|l|}
0.00 \\
\end{tabular} & \begin{tabular}{|l|}
0.00 \\
\end{tabular} & 0.00 \\
\hline \begin{tabular}{|l|}
$72-74$ \\
\end{tabular} & 131.42 & 1866 & 1.69 & 1.12 & \begin{tabular}{l|l}
0.00 \\
\end{tabular} & 0.56 & 0.56 & 4.49 & 0.56 & 2.25 & 0.56 & 0.00 & 0.56 & 0.00 & 0.56 & \begin{tabular}{|l|}
0.00 \\
\end{tabular} & 0.56 & 0.00 \\
\hline \begin{tabular}{|l|}
$76-78$ \\
\end{tabular} & 140.14 & 1857 & 0.00 & 1.11 & 0.00 & 0.00 & 0.00 & 1.11 & 1.11 & 4.44 & 1.11 & 0.00 & 0.00 & 0.00 & \begin{tabular}{|l|}
0.00 \\
\end{tabular} & \begin{tabular}{|l|}
0.00 \\
\end{tabular} & \begin{tabular}{|l|}
0.00 \\
\end{tabular} & $\begin{array}{ll}0.00 \\
\end{array}$ \\
\hline \begin{tabular}{|l|}
$80-82$ \\
\end{tabular} & 148.99 & 1848 & 0.00 & 0.00 & 0.00 & 0.00 & 0.00 & 0.00 & 0.00 & 0.00 & 0.00 & 0.00 & 0.00 & 0.00 & \begin{tabular}{|l|}
0.00 \\
\end{tabular} & 0.00 & 0.00 & 0.00 \\
\hline \begin{tabular}{|l|}
$84-86$ \\
\end{tabular} & 157.97 & 1839 & 0.00 & 0.00 & 0.00 & 0.00 & 0.00 & 0.00 & 0.00 & $\begin{array}{l}0.00 \\
\end{array}$ & 0.00 & 0.00 & 0.00 & 0.00 & \begin{tabular}{|l|}
0.00 \\
\end{tabular} & \begin{tabular}{|l|}
0.00 \\
\end{tabular} & \begin{tabular}{|l|}
0.00 \\
\end{tabular} & 0.00 \\
\hline \begin{tabular}{|l|}
$88-90$ \\
\end{tabular} & 167.08 & 1830 & 0.00 & 0.00 & 0.00 & 0.00 & 0.00 & 0.00 & 0.00 & 0.00 & 0.00 & 0.00 & 0.00 & 0.00 & \begin{tabular}{|l|}
0.00 \\
\end{tabular} & \begin{tabular}{|l|}
0.00 \\
\end{tabular} & 0.00 & 0.00 \\
\hline \begin{tabular}{|l|}
$92-94$ \\
\end{tabular} & 176.32 & 1821 & 0.00 & 0.00 & 0.00 & 0.00 & 0.00 & 0.00 & 0.00 & $\begin{array}{l}0.00 \\
\end{array}$ & 0.00 & 0.00 & 0.00 & 0.00 & \begin{tabular}{|l|}
0.00 \\
\end{tabular} & \begin{tabular}{|l|}
0.00 \\
\end{tabular} & \begin{tabular}{|l|}
0.00 \\
\end{tabular} & 0.00 \\
\hline \begin{tabular}{|l|}
$96-98$ \\
\end{tabular} & 185.68 & 1811 & 0.00 & 0.00 & 0.00 & 0.00 & 0.00 & 0.00 & 0.00 & 0.00 & 0.00 & 0.00 & 0.00 & 0.00 & \begin{tabular}{|l|}
0.00 \\
\end{tabular} & \begin{tabular}{|l|}
0.00 \\
\end{tabular} & 0.00 & 0.00 \\
\hline \begin{tabular}{|l|}
$100-102$ \\
\end{tabular} & 195.17 & 1802 & 0.00 & 0.00 & $\begin{array}{l}0.00 \\
\end{array}$ & 0.00 & 0.00 & 2.63 & 0.00 & 0.00 & 0.00 & 0.00 & 0.00 & 0.00 & \begin{tabular}{|l|}
0.00 \\
\end{tabular} & \begin{tabular}{|l|}
0.00 \\
\end{tabular} & \begin{tabular}{|l|}
0.00 \\
\end{tabular} & 0.00 \\
\hline \begin{tabular}{|l|}
$104-106$ \\
\end{tabular} & 204.79 & 1792 & 0.88 & 0.00 & 0.00 & 0.00 & 0.00 & 0.00 & 0.00 & 0.00 & 0.88 & 0.00 & 0.00 & 0.00 & \begin{tabular}{|l|}
0.00 \\
\end{tabular} & 0.00 & 1.77 & 0.00 \\
\hline \begin{tabular}{|l|}
$108-110$ \\
\end{tabular} & 214.54 & 1782 & 2.63 & 0.00 & 0.00 & 0.00 & 0.00 & 0.00 & 0.00 & 0.00 & 0.00 & 0.00 & 0.00 & 0.00 & \begin{tabular}{|l|}
0.00 \\
\end{tabular} & \begin{tabular}{|l|}
0.00 \\
\end{tabular} & \begin{tabular}{|l|}
0.00 \\
\end{tabular} & 0.00 \\
\hline 112-114 & 224.42 & 1773 & 0.00 & 0.00 & \begin{tabular}{l|l}
0.00 \\
\end{tabular} & 0.00 & 0.00 & 0.00 & 0.00 & 0.00 & 0.00 & 0.00 & 0.00 & 0.00 & 0.00 & \begin{tabular}{|l|}
0.00 \\
\end{tabular} & 0.00 & 0.00 \\
\hline \begin{tabular}{|l|}
$116-118$ \\
\end{tabular} & 234.42 & 1763 & 0.00 & 0.00 & 0.00 & 0.00 & 0.00 & 0.00 & 0.00 & $\mid 10.00$ & 0.00 & 0.00 & 0.00 & 0.00 & \begin{tabular}{|l|}
0.00 \\
\end{tabular} & \begin{tabular}{|l|}
0.00 \\
\end{tabular} & \begin{tabular}{|l|}
0.00 \\
\end{tabular} & 0.00 \\
\hline \begin{tabular}{|l|}
$120-122$ \\
\end{tabular} & 244.55 & 1752 & 0.00 & 0.00 & 0.00 & 0.00 & 0.00 & 0.00 & 0.00 & 0.00 & 0.00 & 0.00 & 0.00 & 0.00 & \begin{tabular}{|l|}
0.00 \\
\end{tabular} & \begin{tabular}{|l|}
1.96 \\
\end{tabular} & \begin{tabular}{|l|}
0.00 \\
\end{tabular} & 0.00 \\
\hline 124-126 & 254.81 & 1742 & 0.00 & 0.00 & 0.00 & 5.26 & 0.00 & 0.00 & 0.00 & 0.00 & 0.00 & 0.00 & 0.00 & 0.00 & 0.00 & 0.00 & 0.00 & 0.00 \\
\hline \begin{tabular}{|l|}
$128-130$ \\
\end{tabular} & 265.20 & 1732 & 0.00 & 0.00 & 0.00 & 0.00 & 0.00 & 0.00 & 0.00 & $\begin{array}{l}0.00 \\
\end{array}$ & 12.00 & 0.00 & 0.00 & 0.00 & \begin{tabular}{|l|}
0.00 \\
\end{tabular} & \begin{tabular}{|l|}
0.00 \\
\end{tabular} & \begin{tabular}{|l|}
0.00 \\
\end{tabular} & 0.00 \\
\hline $132-134$ & 275.72 & \begin{tabular}{ll|}
1721 \\
\end{tabular} & 0.00 & 0.00 & $\begin{array}{l}0.00 \\
\end{array}$ & $\begin{array}{l}0.00 \\
\end{array}$ & $\begin{array}{l}0.00 \\
\end{array}$ & 0.00 & 0.00 & $\begin{array}{l}0.00 \\
\end{array}$ & 0.00 & 0.00 & 0.00 & 0.00 & \begin{tabular}{|l|}
0.00 \\
\end{tabular} & \begin{tabular}{|l|}
0.00 \\
\end{tabular} & 0.00 & 0.00 \\
\hline \begin{tabular}{|l}
$136-138$ \\
\end{tabular} & 286.36 & 1711 & 0.00 & 0.00 & 0.00 & 0.00 & 0.00 & 0.00 & 0.00 & 0.00 & 0.00 & 0.00 & 0.00 & 0.00 & 0.00 & \begin{tabular}{|l|}
0.00 \\
\end{tabular} & 0.00 & 0.00 \\
\hline 140-142 & 297.13 & 1700 & 0.00 & 0.00 & 0.00 & 0.00 & 0.00 & 0.00 & 0.00 & 0.00 & 0.00 & 0.00 & 0.00 & 0.00 & \begin{tabular}{|l|}
0.00 \\
\end{tabular} & \begin{tabular}{|l|}
0.00 \\
\end{tabular} & 0.00 & 0.00 \\
\hline 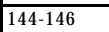 & 308.03 & 1689 & 0.00 & 0.00 & 0.00 & 0.00 & 0.00 & 0.00 & 0.00 & 0.00 & 0.00 & 0.00 & 0.00 & 0.00 & \begin{tabular}{|l|}
0.00 \\
\end{tabular} & \begin{tabular}{|l|}
0.00 \\
\end{tabular} & 6.25 & 0.00 \\
\hline \begin{tabular}{|l}
$148-150$ \\
\end{tabular} & 319.06 & 1678 & 0.00 & 0.00 & 0.00 & 4.55 & 0.00 & 0.00 & 0.00 & 0.00 & 4.55 & 0.00 & 0.00 & 0.00 & \begin{tabular}{|l|}
4.55 \\
\end{tabular} & 0.00 & 0.00 & 0.00 \\
\hline \begin{tabular}{|l|}
$152-154$ \\
\end{tabular} & 330.22 & 1667 & 0.00 & 0.00 & 0.00 & 0.00 & 0.00 & 0.00 & 0.00 & 0.00 & 0.00 & 0.00 & 0.00 & 0.00 & 0.00 & 0.00 & 0.00 & 0.00 \\
\hline \begin{tabular}{|l|}
$156-158$ \\
\end{tabular} & 341.50 & 1655 & 0.00 & 2.78 & 0.00 & 0.00 & 0.00 & 0.00 & 0.00 & 0.00 & 5.56 & 0.00 & 0.00 & 0.00 & \begin{tabular}{|l|}
0.00 \\
\end{tabular} & \begin{tabular}{|l|}
2.78 \\
\end{tabular} & 0.00 & 0.00 \\
\hline \begin{tabular}{|l|}
$160-162$ \\
\end{tabular} & 350.05 & 1647 & 0.00 & 2.56 & 0.00 & 0.00 & 0.00 & 0.00 & 0.00 & 0.00 & 0.00 & 0.00 & 0.00 & 0.00 & \begin{tabular}{|l|}
0.00 \\
\end{tabular} & \begin{tabular}{|l|}
0.00 \\
\end{tabular} & 0.00 & 0.00 \\
\hline \begin{tabular}{|l|}
$164-166$ \\
\end{tabular} & 364.45 & 1633 & 0.00 & 0.00 & 0.00 & 0.00 & 0.00 & 0.00 & 0.00 & $\begin{array}{l}0.00 \\
\end{array}$ & 0.00 & 0.00 & 0.00 & 0.00 & \begin{tabular}{|l|}
4.26 \\
\end{tabular} & \begin{tabular}{|l|}
0.00 \\
\end{tabular} & \begin{tabular}{|l|}
0.00 \\
\end{tabular} & 0.00 \\
\hline $168-170$ & 376.12 & 1621 & 0.00 & 0.00 & 0.00 & 0.00 & 0.00 & 4.55 & 0.00 & 0.00 & 0.00 & 0.00 & 0.00 & 0.00 & 0.00 & 0.00 & 0.00 & 0.00 \\
\hline \begin{tabular}{|l|}
$172-174$ \\
\end{tabular} & 387.92 & 1609 & 0.00 & 0.00 & $\begin{array}{l}0.00 \\
\end{array}$ & 0.00 & $\begin{array}{l}0.00 \\
\end{array}$ & 7.14 & 0.00 & $\begin{array}{l}0.00 \\
\end{array}$ & 0.00 & 0.00 & 0.00 & 0.00 & \begin{tabular}{|l|}
0.00 \\
\end{tabular} & \begin{tabular}{|l|}
0.00 \\
\end{tabular} & \begin{tabular}{|l|}
0.00 \\
\end{tabular} & 0.00 \\
\hline \begin{tabular}{|l|}
$176-178$ \\
\end{tabular} & 399.84 & 1597 & 0.00 & 0.00 & 0.00 & 0.00 & 0.00 & 0.00 & 0.00 & 0.00 & 0.00 & 0.00 & 0.00 & 0.00 & 0.00 & \begin{tabular}{|l|}
0.00 \\
\end{tabular} & 0.00 & 0.00 \\
\hline \begin{tabular}{|l|}
$180-182$ \\
\end{tabular} & 411.89 & 1585 & 0.00 & 0.00 & $\begin{array}{l}0.00 \\
\end{array}$ & 0.00 & 0.00 & 0.00 & 0.00 & $\begin{array}{l}0.00 \\
\end{array}$ & 0.00 & 0.00 & 0.00 & 0.00 & \begin{tabular}{|l|}
0.00 \\
\end{tabular} & \begin{tabular}{|l|}
0.00 \\
\end{tabular} & \begin{tabular}{|l|}
0.00 \\
\end{tabular} & 0.00 \\
\hline 184-186 & 424.07 & 1573 & 0.00 & 0.00 & 0.00 & 0.00 & 0.00 & 0.00 & 0.00 & 0.00 & 0.00 & 0.00 & 0.00 & 3.03 & \begin{tabular}{|l|}
0.00 \\
\end{tabular} & \begin{tabular}{|l|}
0.00 \\
\end{tabular} & 0.00 & 0.00 \\
\hline \begin{tabular}{|l|}
$188-190$ \\
\end{tabular} & 436.38 & 1561 & 0.00 & 0.00 & 0.00 & 7.41 & 0.00 & 0.00 & 3.70 & $\begin{array}{l}0.00 \\
\end{array}$ & 0.00 & 0.00 & 0.00 & 0.00 & \begin{tabular}{|l|}
0.00 \\
\end{tabular} & \begin{tabular}{|l|}
0.00 \\
\end{tabular} & \begin{tabular}{|l|}
0.00 \\
\end{tabular} & 0.00 \\
\hline \begin{tabular}{|l|}
$192-194$ \\
\end{tabular} & 448.82 & 1548 & 0.00 & 0.00 & 2.50 & 0.00 & 0.00 & 0.00 & 0.00 & 2.50 & 0.00 & 0.00 & 0.00 & 0.00 & \begin{tabular}{|l|}
0.00 \\
\end{tabular} & \begin{tabular}{|l|}
0.00 \\
\end{tabular} & 0.00 & 0.00 \\
\hline \begin{tabular}{|l|}
$196-198$ \\
\end{tabular} & 461.38 & 1536 & 0.00 & 0.00 & 0.00 & 0.00 & 0.00 & 0.00 & 0.00 & 0.00 & 0.00 & 0.00 & 0.00 & 7.69 & \begin{tabular}{|l|}
0.00 \\
\end{tabular} & \begin{tabular}{|l|}
0.00 \\
\end{tabular} & \begin{tabular}{|l|}
0.00 \\
\end{tabular} & 0.00 \\
\hline $200-202$ & 474.07 & 1523 & 0.00 & 0.00 & 0.00 & 0.00 & 0.00 & 0.00 & 0.00 & 0.00 & 0.00 & 0.00 & 0.00 & 0.00 & \begin{tabular}{|l|}
0.00 \\
\end{tabular} & 0.00 & 0.00 & 0.00 \\
\hline $204-206$ & 486.89 & 1510 & 0.00 & 0.00 & 0.00 & 0.00 & 0.00 & $\begin{array}{l}0.00 \\
\end{array}$ & 0.00 & 0.00 & 0.00 & $\begin{array}{l}0.00 \\
\end{array}$ & $\begin{array}{l}0.00 \\
\end{array}$ & 22.22 & \begin{tabular}{|l|}
0.00 \\
\end{tabular} & \begin{tabular}{|l|}
0.00 \\
\end{tabular} & \begin{tabular}{|l|}
0.00 \\
\end{tabular} & 0.00 \\
\hline \begin{tabular}{|l|}
$208-210$ \\
\end{tabular} & \begin{tabular}{|l|}
499.84 \\
\end{tabular} & \begin{tabular}{|l|}
1497 \\
\end{tabular} & \begin{tabular}{l|}
0.00 \\
\end{tabular} & \begin{tabular}{l|}
0.00 \\
\end{tabular} & $\begin{array}{l}0.00 \\
\end{array}$ & $\begin{array}{l}0.00 \\
\end{array}$ & \begin{tabular}{l|}
0.00 \\
\end{tabular} & \begin{tabular}{l|}
0.00 \\
\end{tabular} & 0.00 & 0.00 & 0.00 & $\begin{array}{l}0.00 \\
\end{array}$ & $\begin{array}{l}0.00 \\
\end{array}$ & 8.00 & \begin{tabular}{|l|}
0.00 \\
\end{tabular} & \begin{tabular}{|l|}
0.00 \\
\end{tabular} & \begin{tabular}{|l|}
0.00 \\
\end{tabular} & 0.00 \\
\hline $212-214$ & 512.92 & 1484 & 0.00 & 0.00 & \begin{tabular}{l|l}
0.00 \\
\end{tabular} & 0.00 & 0.00 & 0.00 & 0.00 & \begin{tabular}{l|l}
0.00 \\
\end{tabular} & 0.00 & 0.00 & 0.00 & 7.32 & 0.00 & 0.00 & 0.00 & 0.00 \\
\hline $216-218$ & 526.12 & 1471 & 0.00 & 0.00 & 0.00 & 0.00 & 0.00 & 0.00 & 0.00 & 0.00 & 0.00 & 0.00 & 0.00 & 16.00 & 0.00 & 0.00 & 0.00 & 0.00 \\
\hline $220-222$ & 539.45 & 1458 & 0.00 & 0.00 & 0.00 & 0.00 & 0.00 & 0.00 & 0.00 & 0.00 & 0.00 & 0.00 & 0.00 & 0.00 & \begin{tabular}{|l|}
6.67 \\
\end{tabular} & \begin{tabular}{|l|}
0.00 \\
\end{tabular} & 0.00 & 0.00 \\
\hline \begin{tabular}{|l|}
$224-226$ \\
\end{tabular} & 552.91 & 1444 & 0.00 & 0.00 & 0.00 & 7.69 & 0.00 & 0.00 & 0.00 & $\begin{array}{l}0.00 \\
\end{array}$ & 0.00 & 0.00 & 0.00 & 0.00 & \begin{tabular}{|l|}
0.00 \\
\end{tabular} & \begin{tabular}{|l|}
0.00 \\
\end{tabular} & \begin{tabular}{|l|}
0.00 \\
\end{tabular} & 0.00 \\
\hline
\end{tabular}




\begin{tabular}{|c|c|c|c|c|c|c|c|c|c|c|c|c|c|c|c|c|c|c|c|c|}
\hline Depth (cm) & Years (B.P.) & Age Model Year & 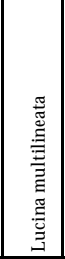 & 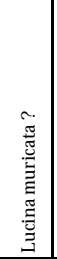 & $\begin{array}{l}\text { है } \\
\Sigma \\
\Sigma\end{array}$ & 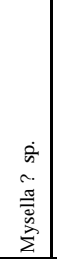 & $\begin{array}{l}\dot{0} \\
\frac{0}{0} \\
\overline{0} \\
\vec{\Sigma} \\
\dot{\Sigma}\end{array}$ & 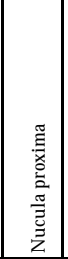 & 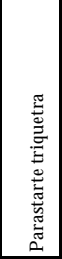 & 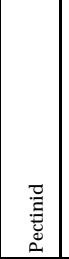 & $\begin{array}{l}\dot{a} \\
\frac{5}{2} \\
\frac{5}{2}\end{array}$ & 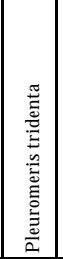 & $\begin{array}{l}0 \\
0 \\
\frac{\pi}{8} \\
8 \\
\frac{1}{2} \\
\frac{0}{0} \\
0\end{array}$ & 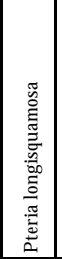 & $\begin{array}{l}\dot{0} \\
0 \\
0 \\
\tilde{n} \\
\omega \\
n\end{array}$ & $\begin{array}{l}\frac{0}{0} \\
0 \\
\frac{0}{5} \\
\underline{0} \\
\end{array}$ & 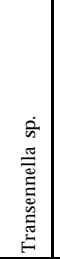 & $\begin{array}{l}\frac{n}{0} \\
\frac{0}{0} \\
\frac{0}{0} \\
\frac{0}{0} \\
\frac{0}{0} \\
\frac{0}{5} \\
\frac{0}{5} \\
\frac{0}{5}\end{array}$ & 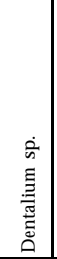 & 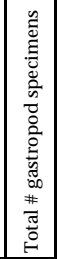 \\
\hline $0-2$ & 0.00 & $\begin{array}{l}1997 \\
\end{array}$ & 0.00 & \begin{tabular}{|l|}
0.00 \\
\end{tabular} & 0.00 & \begin{tabular}{|l|}
1.50 \\
\end{tabular} & 0.00 & 2.26 & 0.00 & 0.00 & 0.00 & \begin{tabular}{|l|}
0.00 \\
\end{tabular} & 0.00 & 2.26 & \begin{tabular}{|l|l|}
0.00 \\
\end{tabular} & \begin{tabular}{|l|}
0.00 \\
\end{tabular} & $\begin{array}{l}1.50 \\
\end{array}$ & 0.00 & \begin{tabular}{|l|}
0.00 \\
\end{tabular} & 133 \\
\hline $4-6$ & 2.69 & 1994 & 0.00 & \begin{tabular}{|l|}
0.00 \\
\end{tabular} & 0.00 & $\begin{array}{ll}0.00 \\
\end{array}$ & $\begin{array}{ll}0.00 \\
\end{array}$ & 2.78 & 0.00 & 0.00 & 0.00 & \begin{tabular}{|l|}
0.00 \\
\end{tabular} & $\begin{array}{l}0.00 \\
\end{array}$ & \begin{tabular}{|l|}
0.00 \\
\end{tabular} & \begin{tabular}{|l|l|}
0.00 \\
\end{tabular} & \begin{tabular}{|l|}
0.00 \\
\end{tabular} & 0.00 & 2.78 & \begin{tabular}{|l|}
0.00 \\
\end{tabular} & 36 \\
\hline $8-10$ & 9.24 & 1988 & 0.00 & \begin{tabular}{|l|}
0.00 \\
\end{tabular} & 0.00 & 0.00 & 0.00 & 0.00 & 0.00 & 0.00 & 0.00 & \begin{tabular}{|l|}
0.00 \\
\end{tabular} & 0.00 & 3.45 & \begin{tabular}{|l|l|}
0.00 \\
\end{tabular} & \begin{tabular}{|l|l|}
1.72 \\
\end{tabular} & 3.45 & 0.00 & \begin{tabular}{|l|}
0.00 \\
\end{tabular} & 58 \\
\hline 12-14 & 15.92 & 1981 & 0.00 & \begin{tabular}{|l|}
0.00 \\
\end{tabular} & 0.00 & 0.00 & \begin{tabular}{|l|}
0.00 \\
\end{tabular} & 0.00 & 0.00 & 0.00 & 0.00 & \begin{tabular}{|l|}
0.00 \\
\end{tabular} & 0.00 & \begin{tabular}{|l|}
0.00 \\
\end{tabular} & \begin{tabular}{|l}
0.00 \\
\end{tabular} & \begin{tabular}{|l|}
0.00 \\
\end{tabular} & \begin{tabular}{|l|}
0.00 \\
\end{tabular} & 0.00 & \begin{tabular}{|l|}
0.00 \\
\end{tabular} & 25 \\
\hline 16-18 & 22.72 & 1974 & 0.00 & \begin{tabular}{|l|}
0.00 \\
\end{tabular} & 0.00 & \begin{tabular}{|l|}
5.71 \\
\end{tabular} & 0.00 & 3.81 & 0.00 & 0.00 & 0.00 & \begin{tabular}{|l|}
0.00 \\
\end{tabular} & 0.00 & \begin{tabular}{|l|}
0.95 \\
\end{tabular} & \begin{tabular}{|l|l|}
0.00 \\
\end{tabular} & \begin{tabular}{|l|}
0.00 \\
\end{tabular} & \begin{tabular}{|l|l|}
0.95 \\
\end{tabular} & 0.00 & \begin{tabular}{|l|}
0.00 \\
\end{tabular} & 105 \\
\hline $20-22$ & 29.65 & 1967 & 0.00 & \begin{tabular}{|l|}
0.00 \\
\end{tabular} & 0.54 & \begin{tabular}{|l|}
0.54 \\
\end{tabular} & 0.00 & 0.54 & 0.00 & 0.00 & 2.72 & \begin{tabular}{|l|}
0.00 \\
\end{tabular} & 0.00 & \begin{tabular}{|l|}
1.09 \\
\end{tabular} & \begin{tabular}{|l|l|}
0.00 \\
\end{tabular} & \begin{tabular}{|l|}
0.00 \\
\end{tabular} & \begin{tabular}{ll|}
0.00 \\
\end{tabular} & 0.00 & \begin{tabular}{|l|}
0.00 \\
\end{tabular} & 184 \\
\hline $24-26$ & 36.71 & 1960 & 0.76 & \begin{tabular}{|l|}
0.00 \\
\end{tabular} & 3.03 & 0.00 & 0.00 & 3.03 & 0.00 & 0.00 & 3.03 & \begin{tabular}{|l|}
0.00 \\
\end{tabular} & \begin{tabular}{|l|}
0.00 \\
\end{tabular} & \begin{tabular}{|l|}
2.27 \\
\end{tabular} & \begin{tabular}{|l}
0.00 \\
\end{tabular} & \begin{tabular}{|l|}
0.76 \\
\end{tabular} & \begin{tabular}{|l|}
0.00 \\
\end{tabular} & 1.52 & \begin{tabular}{|l|}
0.00 \\
\end{tabular} & 132 \\
\hline $28-30$ & 43.90 & 1953 & 0.00 & \begin{tabular}{|l|}
0.00 \\
\end{tabular} & 3.96 & 0.00 & \begin{tabular}{|l|}
0.99 \\
\end{tabular} & 3.96 & 0.00 & 0.00 & 0.00 & \begin{tabular}{|l|}
0.00 \\
\end{tabular} & \begin{tabular}{|l|}
1.98 \\
\end{tabular} & \begin{tabular}{|l|}
1.98 \\
\end{tabular} & 0.00 & \begin{tabular}{|l|}
2.97 \\
\end{tabular} & 0.00 & 0.99 & 0.00 & 101 \\
\hline $32-34$ & 51.22 & 1946 & 0.00 & \begin{tabular}{|l|}
0.00 \\
\end{tabular} & 0.00 & 0.00 & 0.00 & 4.00 & 0.00 & 0.00 & 0.00 & \begin{tabular}{|l|}
0.00 \\
\end{tabular} & 0.00 & \begin{tabular}{|l|l|}
5.33 \\
\end{tabular} & 1.33 & \begin{tabular}{|l|}
0.00 \\
\end{tabular} & \begin{tabular}{|l|l|}
1.33 \\
\end{tabular} & 0.00 & \begin{tabular}{|l|}
0.00 \\
\end{tabular} & 75 \\
\hline $36-38$ & 58.66 & 1938 & 0.00 & \begin{tabular}{|l|}
0.00 \\
\end{tabular} & 5.56 & 0.00 & 0.00 & 3.70 & 0.00 & 0.93 & 0.00 & \begin{tabular}{|l|}
0.00 \\
\end{tabular} & 0.00 & \begin{tabular}{|l|}
0.00 \\
\end{tabular} & 0.00 & 3.70 & \begin{tabular}{l|l|}
1.85 \\
\end{tabular} & 3.70 & \begin{tabular}{|l|}
0.00 \\
\end{tabular} & 108 \\
\hline $40-42$ & 66.23 & 1931 & 0.00 & \begin{tabular}{|l|}
0.00 \\
\end{tabular} & 0.00 & \begin{tabular}{|l|}
0.00 \\
\end{tabular} & \begin{tabular}{|l|}
0.00 \\
\end{tabular} & 1.41 & 0.00 & 0.00 & 0.00 & \begin{tabular}{|l|l|}
0.00 \\
\end{tabular} & \begin{tabular}{|l|}
0.00 \\
\end{tabular} & \begin{tabular}{|l|}
1.41 \\
\end{tabular} & \begin{tabular}{|l|l}
0.00 \\
\end{tabular} & \begin{tabular}{|l|}
2.82 \\
\end{tabular} & \begin{tabular}{|l|}
2.82 \\
\end{tabular} & 7.04 & \begin{tabular}{|l|}
0.00 \\
\end{tabular} & 71 \\
\hline \begin{tabular}{|l|l|}
$44-46$ \\
\end{tabular} & $\begin{array}{l}73.93 \\
\end{array}$ & 1923 & 0.00 & \begin{tabular}{|l|l|}
0.00 \\
\end{tabular} & 0.00 & $\begin{array}{l}0.00 \\
\end{array}$ & $\begin{array}{l}0.00 \\
\end{array}$ & 4.35 & 0.00 & $\begin{array}{l}0.00 \\
\end{array}$ & 0.00 & \begin{tabular}{|l|l|}
0.00 \\
\end{tabular} & \begin{tabular}{|l|l|}
4.35 \\
\end{tabular} & \begin{tabular}{|l|l|}
0.00 \\
\end{tabular} & \begin{tabular}{|l|l}
0.00 \\
\end{tabular} & \begin{tabular}{|l|l|}
0.00 \\
\end{tabular} & \begin{tabular}{|l|}
0.00 \\
\end{tabular} & 4.35 & \begin{tabular}{|l|}
0.00 \\
\end{tabular} & 23 \\
\hline \begin{tabular}{|l|l}
$48-50$ \\
\end{tabular} & 81.76 & $\begin{array}{l}1915 \\
\end{array}$ & 0.00 & \begin{tabular}{|l|}
0.00 \\
\end{tabular} & 0.00 & \begin{tabular}{|l|}
0.00 \\
\end{tabular} & \begin{tabular}{|l|}
0.00 \\
\end{tabular} & 0.00 & 0.00 & 0.00 & 0.00 & \begin{tabular}{|l|l|}
0.00 \\
\end{tabular} & \begin{tabular}{|l|}
0.00 \\
\end{tabular} & \begin{tabular}{|l|}
0.00 \\
\end{tabular} & \begin{tabular}{|l|l}
1.27 \\
\end{tabular} & \begin{tabular}{|l|}
0.00 \\
\end{tabular} & \begin{tabular}{|l|}
2.53 \\
\end{tabular} & 8.86 & \begin{tabular}{|l|}
0.00 \\
\end{tabular} & 79 \\
\hline $52-54$ & 89.72 & 1907 & 0.00 & \begin{tabular}{|l|l|}
0.00 \\
\end{tabular} & 0.00 & \begin{tabular}{|l|}
0.00 \\
\end{tabular} & \begin{tabular}{|l|l|l|}
0.00 \\
\end{tabular} & 0.00 & 0.00 & \begin{tabular}{l|l}
0.00 \\
\end{tabular} & 0.00 & \begin{tabular}{|l|l|}
0.00 \\
\end{tabular} & \begin{tabular}{|l|l|l|}
0.00 \\
\end{tabular} & \begin{tabular}{|l|l|}
0.00 \\
\end{tabular} & \begin{tabular}{|l|l}
0.00 \\
\end{tabular} & \begin{tabular}{|l|l}
4.17 \\
\end{tabular} & \begin{tabular}{|l|}
0.00 \\
\end{tabular} & 4.17 & \begin{tabular}{|l|l|l|}
0.0 \\
\end{tabular} & 48 \\
\hline $56-58$ & 97.80 & \begin{tabular}{|l|l|}
1899 \\
\end{tabular} & 111.76 & \begin{tabular}{|l|}
0.00 \\
\end{tabular} & 0.00 & \begin{tabular}{|l|}
0.00 \\
\end{tabular} & \begin{tabular}{|l|}
0.00 \\
\end{tabular} & 0.00 & 0.00 & \begin{tabular}{l|l}
0.00 \\
\end{tabular} & \begin{tabular}{|l|l|}
11.76 \\
\end{tabular} & \begin{tabular}{|l|}
0.00 \\
\end{tabular} & \begin{tabular}{|l|}
0.00 \\
\end{tabular} & \begin{tabular}{|l|}
0.00 \\
\end{tabular} & \begin{tabular}{|l|l}
0.00 \\
\end{tabular} & \begin{tabular}{|l|l}
0.00 \\
\end{tabular} & \begin{tabular}{|l|}
0.00 \\
\end{tabular} & 5.88 & \begin{tabular}{|l|}
0.00 \\
\end{tabular} & 17 \\
\hline $60-62$ & 106.01 & 1891 & 0.00 & \begin{tabular}{|l|}
0.00 \\
\end{tabular} & 0.00 & \begin{tabular}{|l|}
0.00 \\
\end{tabular} & \begin{tabular}{|l|}
0.00 \\
\end{tabular} & \begin{tabular}{|l|}
8.33 \\
\end{tabular} & 0.00 & \begin{tabular}{l|l}
0.00 \\
\end{tabular} & 4.17 & \begin{tabular}{|l|}
0.00 \\
\end{tabular} & \begin{tabular}{|l|}
0.00 \\
\end{tabular} & \begin{tabular}{|l|l|}
4.17 \\
\end{tabular} & \begin{tabular}{|l|}
0.00 \\
\end{tabular} & \begin{tabular}{|l|}
0.00 \\
\end{tabular} & \begin{tabular}{|l|}
0.00 \\
\end{tabular} & $\begin{array}{l}4.17 \\
\end{array}$ & \begin{tabular}{|l|}
0.00 \\
\end{tabular} & 24 \\
\hline $64-66$ & 114.35 & 1883 & \begin{tabular}{|l|l|}
14.29 \\
\end{tabular} & \begin{tabular}{|l|l|}
0.00 \\
\end{tabular} & 0.00 & $\begin{array}{l}0.00 \\
\end{array}$ & $\begin{array}{l}0.00 \\
\end{array}$ & 0.00 & 0.00 & $\begin{array}{l}0.00 \\
\end{array}$ & 0.00 & \begin{tabular}{|l|l|}
0.00 \\
\end{tabular} & $\begin{array}{l}0.00 \\
\end{array}$ & \begin{tabular}{|l|l|}
0.00 \\
\end{tabular} & \begin{tabular}{|l|l}
0.00 \\
\end{tabular} & \begin{tabular}{|l|}
0.00 \\
\end{tabular} & \begin{tabular}{|l|}
0.00 \\
\end{tabular} & \begin{tabular}{|l|l}
28.57 \\
\end{tabular} & \begin{tabular}{|l|l|}
0.00 \\
\end{tabular} & 7 \\
\hline \begin{tabular}{|l|}
$68-70$ \\
\end{tabular} & 122.82 & \begin{tabular}{|c|}
1874 \\
\end{tabular} & 0.00 & \begin{tabular}{|l|}
0.00 \\
\end{tabular} & 0.00 & \begin{tabular}{|l|}
0.00 \\
\end{tabular} & \begin{tabular}{|l|}
0.00 \\
\end{tabular} & 0.00 & 0.00 & 0.00 & 0.00 & \begin{tabular}{|l|l|}
0.00 \\
\end{tabular} & \begin{tabular}{|l|}
0.00 \\
\end{tabular} & \begin{tabular}{|l|}
0.00 \\
\end{tabular} & \begin{tabular}{|l}
0.00 \\
\end{tabular} & \begin{tabular}{|l|}
0.00 \\
\end{tabular} & \begin{tabular}{|l|}
0.00 \\
\end{tabular} & 0.00 & \begin{tabular}{|l|}
0.00 \\
\end{tabular} & 7 \\
\hline \begin{tabular}{|l|}
$72-74$ \\
\end{tabular} & \begin{tabular}{ll|}
131.42 \\
\end{tabular} & $\begin{array}{l}1866 \\
\end{array}$ & 0.00 & \begin{tabular}{|l|}
0.00 \\
\end{tabular} & $\begin{array}{l}0.00 \\
\end{array}$ & \begin{tabular}{|l|}
0.00 \\
\end{tabular} & \begin{tabular}{|l|l|}
1.12 \\
\end{tabular} & 1.69 & 0.00 & $\begin{array}{ll}0.00 \\
\end{array}$ & $\begin{array}{l}3.93 \\
\end{array}$ & \begin{tabular}{|l|l|}
0.00 \\
\end{tabular} & \begin{tabular}{|l|}
0.00 \\
\end{tabular} & \begin{tabular}{|l|}
2.25 \\
\end{tabular} & \begin{tabular}{|l|l}
0.00 \\
\end{tabular} & \begin{tabular}{|l|}
2.81 \\
\end{tabular} & \begin{tabular}{|l|}
0.00 \\
\end{tabular} & 1.12 & \begin{tabular}{|l|}
0.00 \\
\end{tabular} & 178 \\
\hline 76-78 & 140.14 & \begin{tabular}{|l|l|}
1857 \\
\end{tabular} & 0.00 & \begin{tabular}{|l|}
0.00 \\
\end{tabular} & 0.00 & \begin{tabular}{|l|}
0.00 \\
\end{tabular} & \begin{tabular}{|l|l|}
1.11 \\
\end{tabular} & 0.00 & 0.00 & $\begin{array}{ll}0.00 \\
\end{array}$ & 2.22 & \begin{tabular}{|l|}
0.00 \\
\end{tabular} & \begin{tabular}{|l|}
0.00 \\
\end{tabular} & \begin{tabular}{|l|l|}
1.11 \\
\end{tabular} & \begin{tabular}{|l|l}
0.00 \\
\end{tabular} & \begin{tabular}{|l|}
0.00 \\
\end{tabular} & \begin{tabular}{|l|}
0.00 \\
\end{tabular} & 0.00 & \begin{tabular}{|l|}
0.00 \\
\end{tabular} & 90 \\
\hline $80-82$ & 148.99 & \begin{tabular}{|c|}
1848 \\
\end{tabular} & 2.38 & \begin{tabular}{|l|}
0.00 \\
\end{tabular} & 0.00 & \begin{tabular}{|l|}
0.00 \\
\end{tabular} & \begin{tabular}{|l|}
0.00 \\
\end{tabular} & 2.38 & 0.00 & \begin{tabular}{l|l}
0.00 \\
\end{tabular} & 0.00 & \begin{tabular}{|l|}
0.00 \\
\end{tabular} & \begin{tabular}{|l|}
0.00 \\
\end{tabular} & \begin{tabular}{|l|}
0.00 \\
\end{tabular} & \begin{tabular}{|l}
0.00 \\
\end{tabular} & \begin{tabular}{|l|}
1.19 \\
\end{tabular} & \begin{tabular}{|l|}
0.00 \\
\end{tabular} & 0.00 & \begin{tabular}{|l|}
0.00 \\
\end{tabular} & 84 \\
\hline $84-86$ & 157.97 & 1839 & 0.00 & \begin{tabular}{|l|}
0.00 \\
\end{tabular} & 0.00 & \begin{tabular}{|l|}
0.00 \\
\end{tabular} & \begin{tabular}{|l|}
0.00 \\
\end{tabular} & 0.00 & 0.00 & \begin{tabular}{l|l}
0.00 \\
\end{tabular} & 0.00 & \begin{tabular}{|l|l|}
0.00 \\
\end{tabular} & \begin{tabular}{|l|}
0.00 \\
\end{tabular} & \begin{tabular}{|l|}
0.00 \\
\end{tabular} & \begin{tabular}{|l|l}
0.00 \\
\end{tabular} & \begin{tabular}{|l|}
0.00 \\
\end{tabular} & \begin{tabular}{|l|}
0.00 \\
\end{tabular} & 0.00 & \begin{tabular}{|l|}
0.00 \\
\end{tabular} & 19 \\
\hline $88-90$ & 167.08 & 1830 & 0.00 & \begin{tabular}{|l|}
0.00 \\
\end{tabular} & 0.00 & \begin{tabular}{|l|}
0.00 \\
\end{tabular} & \begin{tabular}{|l|}
0.00 \\
\end{tabular} & 0.00 & 0.00 & $\begin{array}{l}0.00 \\
\end{array}$ & $\begin{array}{l}3.23 \\
\end{array}$ & \begin{tabular}{|l|l|}
0.00 \\
\end{tabular} & \begin{tabular}{|l|}
0.00 \\
\end{tabular} & \begin{tabular}{|l|l|}
0.00 \\
\end{tabular} & \begin{tabular}{|l|l}
0.00 \\
\end{tabular} & \begin{tabular}{|l|}
0.00 \\
\end{tabular} & \begin{tabular}{|l|}
0.00 \\
\end{tabular} & 0.00 & \begin{tabular}{|l|}
0.00 \\
\end{tabular} & 31 \\
\hline \begin{tabular}{|c|}
$92-94$ \\
\end{tabular} & 176.32 & 1821 & 0.00 & \begin{tabular}{|l|}
0.00 \\
\end{tabular} & $\begin{array}{l}3.45 \\
\end{array}$ & \begin{tabular}{|l|}
0.00 \\
\end{tabular} & \begin{tabular}{|l|l|}
0.00 \\
\end{tabular} & $\begin{array}{l}0.00 \\
\end{array}$ & 0.00 & \begin{tabular}{l|l}
0.00 \\
\end{tabular} & 0.00 & \begin{tabular}{|l|l|}
0.00 \\
\end{tabular} & \begin{tabular}{|l|}
0.00 \\
\end{tabular} & \begin{tabular}{|l|l|}
0.00 \\
\end{tabular} & \begin{tabular}{|l|l}
0.00 \\
\end{tabular} & \begin{tabular}{|l|}
0.00 \\
\end{tabular} & \begin{tabular}{|l|}
0.00 \\
\end{tabular} & 0.00 & \begin{tabular}{|l|l|}
0.00 \\
\end{tabular} & 29 \\
\hline \begin{tabular}{|c|}
$96-98$ \\
\end{tabular} & 185.68 & \begin{tabular}{|l|l|}
1811 \\
\end{tabular} & 0.00 & \begin{tabular}{|l|}
0.00 \\
\end{tabular} & $\begin{array}{l}0.00 \\
\end{array}$ & \begin{tabular}{|l|}
0.00 \\
\end{tabular} & \begin{tabular}{|l|}
0.00 \\
\end{tabular} & 0.00 & 0.00 & \begin{tabular}{l|l}
0.00 \\
\end{tabular} & 0.00 & \begin{tabular}{|l|l|}
0.00 \\
\end{tabular} & \begin{tabular}{|l|}
0.00 \\
\end{tabular} & \begin{tabular}{|l|l|}
0.00 \\
\end{tabular} & \begin{tabular}{|l|l}
0.00 \\
\end{tabular} & \begin{tabular}{|l|}
0.00 \\
\end{tabular} & \begin{tabular}{|l|}
0.00 \\
\end{tabular} & 0.00 & \begin{tabular}{|l|}
0.00 \\
\end{tabular} & 23 \\
\hline 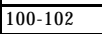 & $\begin{array}{l}195.17 \\
\end{array}$ & 1802 & 0.00 & \begin{tabular}{|l|}
0.00 \\
\end{tabular} & 0.00 & \begin{tabular}{|l|}
0.00 \\
\end{tabular} & \begin{tabular}{|l|}
0.00 \\
\end{tabular} & 0.00 & 0.00 & 0 & 0.00 & \begin{tabular}{|l|l|}
0.00 \\
\end{tabular} & \begin{tabular}{|l|}
0.00 \\
\end{tabular} & \begin{tabular}{|l|l|}
0.00 \\
\end{tabular} & \begin{tabular}{|l|l}
0.00 \\
\end{tabular} & \begin{tabular}{|l|}
0.00 \\
\end{tabular} & \begin{tabular}{|l|l|}
0.00 \\
\end{tabular} & 0.00 & \begin{tabular}{|l|}
0.00 \\
\end{tabular} & 76 \\
\hline \begin{tabular}{|l|}
$104-106$ \\
\end{tabular} & 20 & 1792 & 0.00 & \begin{tabular}{|l|}
0.00 \\
\end{tabular} & 0.00 & \begin{tabular}{|l|}
0.00 \\
\end{tabular} & \begin{tabular}{|l|}
0.00 \\
\end{tabular} & $\begin{array}{l}0.88 \\
\end{array}$ & 0.00 & 0.00 & 0.00 & \begin{tabular}{|l|}
0.00 \\
\end{tabular} & $\begin{array}{l}0.00 \\
\end{array}$ & \begin{tabular}{|l|}
0.00 \\
\end{tabular} & \begin{tabular}{|l|l|}
0.00 \\
\end{tabular} & \begin{tabular}{|l|}
0.00 \\
\end{tabular} & \begin{tabular}{|l|}
0.00 \\
\end{tabular} & 0.00 & \begin{tabular}{|l|}
0.00 \\
\end{tabular} & 113 \\
\hline \begin{tabular}{|l}
$108-110$ \\
\end{tabular} & 214.54 & 1782 & 0.00 & \begin{tabular}{|l|}
0.00 \\
\end{tabular} & 0.00 & \begin{tabular}{|l|}
0.00 \\
\end{tabular} & \begin{tabular}{|l|}
0.00 \\
\end{tabular} & 0.00 & 0.00 & 0.00 & 0.00 & \begin{tabular}{|l|}
0.00 \\
\end{tabular} & \begin{tabular}{|l|}
0.00 \\
\end{tabular} & \begin{tabular}{|l|}
0.00 \\
\end{tabular} & \begin{tabular}{|l|}
0.00 \\
\end{tabular} & \begin{tabular}{|l|}
0.00 \\
\end{tabular} & \begin{tabular}{|l|}
0.00 \\
\end{tabular} & 0.00 & \begin{tabular}{|l|}
0.00 \\
\end{tabular} & 38 \\
\hline \begin{tabular}{|l|}
$112-114$ \\
\end{tabular} & 224.42 & \begin{tabular}{|l|l|}
1773 \\
\end{tabular} & 0.00 & \begin{tabular}{|l|}
0.00 \\
\end{tabular} & 0.00 & $\begin{array}{l}0.00 \\
\end{array}$ & $\begin{array}{l}0.00 \\
\end{array}$ & 0.00 & 0.00 & $\begin{array}{ll}0.00 \\
\end{array}$ & 0.00 & \begin{tabular}{|l|}
0.00 \\
\end{tabular} & \begin{tabular}{|l|}
0.00 \\
\end{tabular} & \begin{tabular}{|l|}
0.00 \\
\end{tabular} & \begin{tabular}{|l|}
0.00 \\
\end{tabular} & \begin{tabular}{|l|}
0.00 \\
\end{tabular} & \begin{tabular}{|l|}
0.00 \\
\end{tabular} & 0.00 & $\begin{array}{l}0.00 \\
\end{array}$ & 5 \\
\hline \begin{tabular}{|l|}
$116-118$ \\
\end{tabular} & 2 & 1763 & 0.00 & \begin{tabular}{|l|}
0.00 \\
\end{tabular} & 0.00 & \begin{tabular}{|l|}
0.00 \\
\end{tabular} & \begin{tabular}{|l|}
0.00 \\
\end{tabular} & 0.00 & 0.00 & 0.00 & 0.00 & \begin{tabular}{|l|}
0.00 \\
\end{tabular} & \begin{tabular}{|l|}
0.00 \\
\end{tabular} & \begin{tabular}{|l|}
0.00 \\
\end{tabular} & \begin{tabular}{|l|}
0.00 \\
\end{tabular} & \begin{tabular}{|l|}
0.00 \\
\end{tabular} & \begin{tabular}{|l|}
0.00 \\
\end{tabular} & 0.00 & \begin{tabular}{|l|}
0.00 \\
\end{tabular} & 10 \\
\hline \begin{tabular}{|l}
$120-122$ \\
\end{tabular} & 244.55 & 1752 & 0.00 & \begin{tabular}{|l|}
0.00 \\
\end{tabular} & 0.00 & \begin{tabular}{|l|}
0.00 \\
\end{tabular} & \begin{tabular}{|l|}
0.00 \\
\end{tabular} & 0.00 & 0.00 & 0.00 & 0.00 & \begin{tabular}{|l|}
0.00 \\
\end{tabular} & \begin{tabular}{|l|}
0.00 \\
\end{tabular} & \begin{tabular}{|l|}
0.00 \\
\end{tabular} & \begin{tabular}{|l|}
0.00 \\
\end{tabular} & \begin{tabular}{|l|}
1.96 \\
\end{tabular} & \begin{tabular}{|l|}
0.00 \\
\end{tabular} & 0.00 & \begin{tabular}{|l|}
0.00 \\
\end{tabular} & 102 \\
\hline \begin{tabular}{|l|}
$124-126$ \\
\end{tabular} & 254.81 & $\begin{array}{l}1742 \\
\end{array}$ & 0.00 & \begin{tabular}{|l|}
0.00 \\
\end{tabular} & 0.00 & $\begin{array}{l}0.00 \\
\end{array}$ & \begin{tabular}{|l|}
0.00 \\
\end{tabular} & 0.00 & 0.00 & $\begin{array}{l}0.00 \\
\end{array}$ & 0.00 & \begin{tabular}{|l|l|}
0.00 \\
\end{tabular} & \begin{tabular}{|l|}
0.00 \\
\end{tabular} & \begin{tabular}{|l|l|}
0.00 \\
\end{tabular} & \begin{tabular}{|l|l|}
0.00 \\
\end{tabular} & \begin{tabular}{|l|}
0.00 \\
\end{tabular} & \begin{tabular}{|l|}
0.00 \\
\end{tabular} & 5.26 & \begin{tabular}{|l|}
0.00 \\
\end{tabular} & 19 \\
\hline \begin{tabular}{|l|}
$128-130$ \\
\end{tabular} & 265.20 & 1732 & 0.00 & \begin{tabular}{|l|}
0.00 \\
\end{tabular} & 0.00 & 0.00 & \begin{tabular}{|l|}
0.00 \\
\end{tabular} & 0.00 & 0.00 & \begin{tabular}{l|l}
0.00 \\
\end{tabular} & 0.00 & \begin{tabular}{|l|}
0.00 \\
\end{tabular} & $\begin{array}{l}0.00 \\
\end{array}$ & \begin{tabular}{|l|}
0.00 \\
\end{tabular} & 0.00 & \begin{tabular}{|l|}
0.00 \\
\end{tabular} & \begin{tabular}{|l|}
0.00 \\
\end{tabular} & 8.00 & \begin{tabular}{|l|}
0.00 \\
\end{tabular} & 25 \\
\hline 132-134 & 275.72 & 1721 & 0.00 & \begin{tabular}{|l|}
0.00 \\
\end{tabular} & \begin{tabular}{l|l|}
0.00 \\
\end{tabular} & \begin{tabular}{|c|}
0.00 \\
\end{tabular} & \begin{tabular}{|c|}
0.00 \\
\end{tabular} & 0.00 & 2.44 & \begin{tabular}{l|l}
0.00 \\
\end{tabular} & 0.00 & \begin{tabular}{|l|}
2.44 \\
\end{tabular} & \begin{tabular}{|c|}
0.00 \\
\end{tabular} & \begin{tabular}{|l|}
0.00 \\
\end{tabular} & 0.00 & \begin{tabular}{|l|l|}
4.88 \\
\end{tabular} & \begin{tabular}{|l|}
0.00 \\
\end{tabular} & 0.00 & \begin{tabular}{|l|}
0.00 \\
\end{tabular} & 41 \\
\hline \begin{tabular}{|l}
$136-138$ \\
\end{tabular} & 286.36 & 1711 & 0.00 & \begin{tabular}{|l|}
0.00 \\
\end{tabular} & 0.00 & \begin{tabular}{|l|}
0.00 \\
\end{tabular} & \begin{tabular}{|l|}
0.00 \\
\end{tabular} & 0.00 & \begin{tabular}{|l|l|}
11.11 \\
\end{tabular} & 0.00 & 0.00 & \begin{tabular}{|l|}
0.00 \\
\end{tabular} & \begin{tabular}{|l|}
0.00 \\
\end{tabular} & \begin{tabular}{|l|}
0.00 \\
\end{tabular} & \begin{tabular}{|l|l|}
0.00 \\
\end{tabular} & \begin{tabular}{|l|}
0.00 \\
\end{tabular} & \begin{tabular}{|l|}
0.00 \\
\end{tabular} & 0.00 & \begin{tabular}{|l|}
0.00 \\
\end{tabular} & 9 \\
\hline \begin{tabular}{|l}
$140-142$ \\
\end{tabular} & 297.13 & 1700 & 0.00 & \begin{tabular}{|l|}
0.00 \\
\end{tabular} & 0.00 & \begin{tabular}{|l|}
0.00 \\
\end{tabular} & \begin{tabular}{|l|}
0.00 \\
\end{tabular} & 0.00 & 0.00 & 0.00 & 0.00 & \begin{tabular}{|l|}
0.00 \\
\end{tabular} & \begin{tabular}{|l|}
0.00 \\
\end{tabular} & \begin{tabular}{|l|}
0.00 \\
\end{tabular} & \begin{tabular}{|l|}
0.00 \\
\end{tabular} & \begin{tabular}{|l|}
0.00 \\
\end{tabular} & \begin{tabular}{|l|}
0.00 \\
\end{tabular} & \begin{tabular}{|l|}
14.29 \\
\end{tabular} & \begin{tabular}{|l|}
0.00 \\
\end{tabular} & 14 \\
\hline \begin{tabular}{|l|}
$144-146$ \\
\end{tabular} & 308.03 & 1689 & 0.00 & \begin{tabular}{|l|}
0.00 \\
\end{tabular} & 0.00 & \begin{tabular}{|l|}
0.00 \\
\end{tabular} & \begin{tabular}{|l|}
0.00 \\
\end{tabular} & 0.00 & 0.00 & $\begin{array}{ll}0.00 \\
\end{array}$ & 0.00 & \begin{tabular}{|l|}
0.00 \\
\end{tabular} & \begin{tabular}{|l|}
0.00 \\
\end{tabular} & \begin{tabular}{|l|}
0.00 \\
\end{tabular} & \begin{tabular}{|l|}
0.00 \\
\end{tabular} & \begin{tabular}{|l|}
0.00 \\
\end{tabular} & \begin{tabular}{|l|}
0.00 \\
\end{tabular} & 6.25 & \begin{tabular}{|l|}
0.00 \\
\end{tabular} & 16 \\
\hline \begin{tabular}{|l|}
$148-150$ \\
\end{tabular} & 319. & 1678 & 0.00 & \begin{tabular}{|l|}
0.00 \\
\end{tabular} & 0.00 & \begin{tabular}{|l|}
0.00 \\
\end{tabular} & \begin{tabular}{|l|}
0.00 \\
\end{tabular} & 0.00 & 0.00 & 0.00 & 0.00 & \begin{tabular}{|l|l|}
0.00 \\
\end{tabular} & \begin{tabular}{|l|}
0.00 \\
\end{tabular} & \begin{tabular}{|l|}
0.00 \\
\end{tabular} & \begin{tabular}{|l|l|}
0.00 \\
\end{tabular} & \begin{tabular}{|l|l|}
0.00 \\
\end{tabular} & \begin{tabular}{|l|}
0.00 \\
\end{tabular} & 9.09 & \begin{tabular}{|l|}
0.00 \\
\end{tabular} & 22 \\
\hline \begin{tabular}{|l|}
$152-154$ \\
\end{tabular} & 330.22 & $\begin{array}{l}1667 \\
\end{array}$ & 0.00 & \begin{tabular}{|l|}
0.00 \\
\end{tabular} & 0.00 & $\begin{array}{l}0.00 \\
\end{array}$ & \begin{tabular}{|l|}
2.86 \\
\end{tabular} & 2.86 & 0.00 & 0.00 & 0.00 & \begin{tabular}{|l|}
0.00 \\
\end{tabular} & \begin{tabular}{|l|}
0.00 \\
\end{tabular} & \begin{tabular}{|l|}
0.00 \\
\end{tabular} & \begin{tabular}{|l|}
0.00 \\
\end{tabular} & \begin{tabular}{|l|}
0.00 \\
\end{tabular} & \begin{tabular}{|l|}
0.00 \\
\end{tabular} & \begin{tabular}{|l|l|}
11.43 \\
\end{tabular} & \begin{tabular}{|l|}
0.00 \\
\end{tabular} & 35 \\
\hline \begin{tabular}{|l}
$156-158$ \\
\end{tabular} & 341.50 & 1655 & 0.00 & \begin{tabular}{|l|}
0.00 \\
\end{tabular} & 0.00 & \begin{tabular}{|l|}
0.00 \\
\end{tabular} & \begin{tabular}{|l|}
1.39 \\
\end{tabular} & 1.39 & 0.00 & \begin{tabular}{l|l}
0.00 \\
\end{tabular} & 0.00 & \begin{tabular}{|l|}
0.00 \\
\end{tabular} & \begin{tabular}{|l|}
0.00 \\
\end{tabular} & \begin{tabular}{|l|}
0.00 \\
\end{tabular} & \begin{tabular}{|l}
0.00 \\
\end{tabular} & \begin{tabular}{|l|}
0.00 \\
\end{tabular} & \begin{tabular}{|l|}
0.00 \\
\end{tabular} & 4.17 & \begin{tabular}{|l|}
0.00 \\
\end{tabular} & 72 \\
\hline \begin{tabular}{|l}
$160-162$ \\
\end{tabular} & 350.05 & \begin{tabular}{|l|}
1647 \\
\end{tabular} & 0.00 & \begin{tabular}{|l|}
0.00 \\
\end{tabular} & 0.00 & \begin{tabular}{|l|}
0.00 \\
\end{tabular} & \begin{tabular}{|l|}
0.00 \\
\end{tabular} & 1.28 & 0.00 & 0.00 & 0.00 & \begin{tabular}{|l|}
0.00 \\
\end{tabular} & \begin{tabular}{|l|}
0.00 \\
\end{tabular} & \begin{tabular}{|l|}
0.00 \\
\end{tabular} & \begin{tabular}{|l|}
0.00 \\
\end{tabular} & \begin{tabular}{|l|}
2.56 \\
\end{tabular} & \begin{tabular}{|l|}
0.00 \\
\end{tabular} & 2.56 & \begin{tabular}{|l|}
0.00 \\
\end{tabular} & 78 \\
\hline \begin{tabular}{|l|}
$164-166$ \\
\end{tabular} & 364.45 & \begin{tabular}{|l|l|}
1633 \\
\end{tabular} & 0.00 & \begin{tabular}{|l|}
0.00 \\
\end{tabular} & 0.00 & \begin{tabular}{|l|}
0.00 \\
\end{tabular} & \begin{tabular}{|l|}
0.00 \\
\end{tabular} & 0.00 & 0.00 & 0.00 & 0.00 & \begin{tabular}{|l|}
0.00 \\
\end{tabular} & \begin{tabular}{|l|}
0.00 \\
\end{tabular} & \begin{tabular}{|l|}
0.00 \\
\end{tabular} & \begin{tabular}{|l|}
0.00 \\
\end{tabular} & \begin{tabular}{|l|}
0.00 \\
\end{tabular} & \begin{tabular}{|l|}
0.00 \\
\end{tabular} & 0.00 & \begin{tabular}{|l|}
0.00 \\
\end{tabular} & 47 \\
\hline \begin{tabular}{|l|}
$168-170$ \\
\end{tabular} & 31 & 162 & 0.00 & $\begin{array}{ll}0.00 \\
\end{array}$ & 0.00 & \begin{tabular}{|l|}
0.00 \\
\end{tabular} & \begin{tabular}{|l|}
0.00 \\
\end{tabular} & 0.00 & 0.00 & \begin{tabular}{l|}
0.00 \\
\end{tabular} & 0.00 & \begin{tabular}{|l|}
0.00 \\
\end{tabular} & $\begin{array}{l}0.00 \\
\end{array}$ & \begin{tabular}{|l|}
0.00 \\
\end{tabular} & \begin{tabular}{|l|}
0.00 \\
\end{tabular} & \begin{tabular}{|l|}
0.00 \\
\end{tabular} & \begin{tabular}{|l|}
0.00 \\
\end{tabular} & 0.00 & \begin{tabular}{|l|}
0.00 \\
\end{tabular} & 22 \\
\hline \begin{tabular}{|l|}
$172-174$ \\
\end{tabular} & \begin{tabular}{|l|}
387.92 \\
\end{tabular} & 1609 & 0.00 & \begin{tabular}{|l|}
0.00 \\
\end{tabular} & 0.00 & \begin{tabular}{|l|}
0.00 \\
\end{tabular} & \begin{tabular}{|l|}
0.00 \\
\end{tabular} & 0.00 & 0.00 & 0.00 & 0.00 & \begin{tabular}{|l|}
0.00 \\
\end{tabular} & \begin{tabular}{|l|}
0.00 \\
\end{tabular} & \begin{tabular}{|l|}
0.00 \\
\end{tabular} & \begin{tabular}{|l|}
0.00 \\
\end{tabular} & \begin{tabular}{|l|}
0.00 \\
\end{tabular} & \begin{tabular}{|l|}
0.00 \\
\end{tabular} & 0.00 & \begin{tabular}{|l|}
0.00 \\
\end{tabular} & 28 \\
\hline \begin{tabular}{|l}
$176-178$ \\
\end{tabular} & 399.84 & $\begin{array}{l}1597 \\
\end{array}$ & \begin{tabular}{|l|l|}
10.53 \\
\end{tabular} & \begin{tabular}{|l|}
0.00 \\
\end{tabular} & 0.00 & \begin{tabular}{|l|} 
\\
\end{tabular} & \begin{tabular}{|l|}
0.00 \\
\end{tabular} & 0.00 & 0.00 & \begin{tabular}{l|l}
0.00 \\
\end{tabular} & 0.00 & \begin{tabular}{|l|}
0.00 \\
\end{tabular} & \begin{tabular}{|l|}
0.00 \\
\end{tabular} & \begin{tabular}{|l|}
0.00 \\
\end{tabular} & \begin{tabular}{|l|}
0.00 \\
\end{tabular} & \begin{tabular}{|l|}
0.00 \\
\end{tabular} & \begin{tabular}{|l|}
0.00 \\
\end{tabular} & 5.26 & \begin{tabular}{|l|}
0.00 \\
\end{tabular} & 19 \\
\hline \begin{tabular}{|l}
$180-182$ \\
\end{tabular} & & 1585 & \begin{tabular}{|l|}
0.00 \\
\end{tabular} & \begin{tabular}{|l|}
0.00 \\
\end{tabular} & 0.00 & \begin{tabular}{|l|}
0.00 \\
\end{tabular} & \begin{tabular}{|l|}
0.00 \\
\end{tabular} & \begin{tabular}{|r|}
6.67 \\
\end{tabular} & 0.00 & 0.00 & 0.00 & \begin{tabular}{|l|}
0.00 \\
\end{tabular} & \begin{tabular}{|l|}
0.00 \\
\end{tabular} & \begin{tabular}{|l|}
0.00 \\
\end{tabular} & \begin{tabular}{|l|}
0.00 \\
\end{tabular} & \begin{tabular}{|l|}
0.00 \\
\end{tabular} & \begin{tabular}{|l|}
0.00 \\
\end{tabular} & 0.00 & \begin{tabular}{|l|}
0.00 \\
\end{tabular} & 30 \\
\hline \begin{tabular}{|l|}
$184-186$ \\
\end{tabular} & 424.07 & 1573 & 0.00 & \begin{tabular}{|l|}
0.00 \\
\end{tabular} & 0.00 & \begin{tabular}{|l|}
0.00 \\
\end{tabular} & \begin{tabular}{|l|}
0.00 \\
\end{tabular} & 9.09 & 0.00 & 0.00 & 3.03 & \begin{tabular}{|l|}
0.00 \\
\end{tabular} & \begin{tabular}{|l|}
0.00 \\
\end{tabular} & \begin{tabular}{|l|}
0.00 \\
\end{tabular} & \begin{tabular}{|l|}
0.00 \\
\end{tabular} & \begin{tabular}{|l|}
0.00 \\
\end{tabular} & \begin{tabular}{|l|}
0.00 \\
\end{tabular} & 9.09 & \begin{tabular}{|l|}
0.00 \\
\end{tabular} & 33 \\
\hline \begin{tabular}{|l|}
$188-190$ \\
\end{tabular} & 436.38 & 1561 & 0.00 & \begin{tabular}{|l|}
0.00 \\
\end{tabular} & 0.00 & \begin{tabular}{|l|}
0.00 \\
\end{tabular} & $\begin{array}{l}0.00 \\
\end{array}$ & 0.00 & 0.00 & \begin{tabular}{l|l}
0.00 \\
\end{tabular} & 0.00 & \begin{tabular}{|l|}
0.00 \\
\end{tabular} & $\begin{array}{l}0.00 \\
\end{array}$ & \begin{tabular}{|l|}
0.00 \\
\end{tabular} & \begin{tabular}{|l|}
0.00 \\
\end{tabular} & \begin{tabular}{|l|}
0.00 \\
\end{tabular} & \begin{tabular}{|l|}
0.00 \\
\end{tabular} & 3.70 & \begin{tabular}{|l|}
0.00 \\
\end{tabular} & 27 \\
\hline \begin{tabular}{|l|}
$192-194$ \\
\end{tabular} & \begin{tabular}{|l|l|}
448.82 \\
\end{tabular} & 1548 & 0.00 & 2.50 & 0.00 & $\begin{array}{l}0.00 \\
\end{array}$ & $\begin{array}{l}0.00 \\
\end{array}$ & 0.00 & \begin{tabular}{c|}
0.00 \\
\end{tabular} & \begin{tabular}{l|l}
0.00 \\
\end{tabular} & 5.00 & \begin{tabular}{|l|}
0.00 \\
\end{tabular} & 2.50 & \begin{tabular}{|l|}
0.00 \\
\end{tabular} & \begin{tabular}{|l|l|}
0.00 \\
\end{tabular} & \begin{tabular}{|l|}
0.00 \\
\end{tabular} & \begin{tabular}{|l|}
0.00 \\
\end{tabular} & 7.50 & \begin{tabular}{|l|l|}
0.00 \\
\end{tabular} & 40 \\
\hline \begin{tabular}{|l}
$196-198$ \\
\end{tabular} & 461.38 & 1536 & 0.00 & \begin{tabular}{|l|}
0.00 \\
\end{tabular} & 0.00 & \begin{tabular}{|l|}
0.00 \\
\end{tabular} & \begin{tabular}{|l|}
0.00 \\
\end{tabular} & \begin{tabular}{|l|}
15.38 \\
\end{tabular} & 0.00 & \begin{tabular}{l|l}
0.00 \\
\end{tabular} & 0.00 & \begin{tabular}{|l|}
0.00 \\
\end{tabular} & \begin{tabular}{|l|}
0.00 \\
\end{tabular} & \begin{tabular}{|l|}
0.00 \\
\end{tabular} & \begin{tabular}{|l|l|}
0.00 \\
\end{tabular} & \begin{tabular}{|l|}
0.00 \\
\end{tabular} & \begin{tabular}{|l|}
0.00 \\
\end{tabular} & 7.69 & \begin{tabular}{|l|}
0.00 \\
\end{tabular} & 13 \\
\hline \begin{tabular}{|l}
$200-202$ \\
\end{tabular} & \begin{tabular}{|l|l|}
474.07 \\
\end{tabular} & 1523 & $\begin{array}{l}9.09 \\
\end{array}$ & \begin{tabular}{|l|}
0.00 \\
\end{tabular} & 0.00 & \begin{tabular}{|l|}
0.00 \\
\end{tabular} & \begin{tabular}{|l|}
0.00 \\
\end{tabular} & \begin{tabular}{|l|}
0.00 \\
\end{tabular} & 0.00 & \begin{tabular}{|l|}
18.18 \\
\end{tabular} & $\begin{array}{l}9.09 \\
\end{array}$ & \begin{tabular}{|l|}
0.00 \\
\end{tabular} & \begin{tabular}{|l|}
0.00 \\
\end{tabular} & \begin{tabular}{|l|}
0.00 \\
\end{tabular} & \begin{tabular}{|l|}
0.00 \\
\end{tabular} & \begin{tabular}{|l|}
0.00 \\
\end{tabular} & \begin{tabular}{|l|}
0.00 \\
\end{tabular} & 0.00 & \begin{tabular}{|l|}
0.00 \\
\end{tabular} & 11 \\
\hline \begin{tabular}{|l|}
$204-206$ \\
\end{tabular} & 486.89 & 1510 & 0.00 & \begin{tabular}{|l|}
0.00 \\
\end{tabular} & 0.00 & $\begin{array}{l}0.00 \\
\end{array}$ & $\begin{array}{l}0.00 \\
\end{array}$ & 0.00 & 0.00 & 0.00 & \begin{tabular}{|l|}
11.11 \\
\end{tabular} & \begin{tabular}{|l|}
0.00 \\
\end{tabular} & $\begin{array}{l}0.00 \\
\end{array}$ & \begin{tabular}{|l|}
0.00 \\
\end{tabular} & \begin{tabular}{|l|}
0.00 \\
\end{tabular} & \begin{tabular}{|l|}
0.00 \\
\end{tabular} & \begin{tabular}{|l|}
0.00 \\
\end{tabular} & \begin{tabular}{|l|}
11.11 \\
\end{tabular} & \begin{tabular}{|l|}
0.00 \\
\end{tabular} & 9 \\
\hline \begin{tabular}{|l|}
$208-210$ \\
\end{tabular} & 499.84 & \begin{tabular}{|l|l|}
1497 \\
\end{tabular} & 0.00 & \begin{tabular}{|l|}
0.00 \\
\end{tabular} & 0.00 & \begin{tabular}{|c|}
0.00 \\
\end{tabular} & \begin{tabular}{|c|}
0.00 \\
\end{tabular} & 8.00 & 0.00 & 4.00 & 8.00 & \begin{tabular}{|l|}
0.00 \\
\end{tabular} & \begin{tabular}{|c|}
0.00 \\
\end{tabular} & \begin{tabular}{|l|}
0.00 \\
\end{tabular} & \begin{tabular}{|l|}
0.00 \\
\end{tabular} & \begin{tabular}{|l|}
0.00 \\
\end{tabular} & \begin{tabular}{|l|}
0.00 \\
\end{tabular} & 4.00 & \begin{tabular}{|l|}
0.00 \\
\end{tabular} & 25 \\
\hline 212-214 & 512.92 & $\begin{array}{l}1484 \\
\end{array}$ & 2.44 & \begin{tabular}{|l|}
2.44 \\
\end{tabular} & 0.00 & \begin{tabular}{|l|}
0.00 \\
\end{tabular} & \begin{tabular}{|l|}
2.44 \\
\end{tabular} & 0.00 & 0.00 & 2.44 & 2.44 & \begin{tabular}{|l|}
0.00 \\
\end{tabular} & \begin{tabular}{|l|}
0.00 \\
\end{tabular} & \begin{tabular}{|l|}
2.44 \\
\end{tabular} & \begin{tabular}{|l|}
0.00 \\
\end{tabular} & \begin{tabular}{|l|}
0.00 \\
\end{tabular} & \begin{tabular}{|l|}
0.00 \\
\end{tabular} & 4.88 & \begin{tabular}{|l|}
2.44 \\
\end{tabular} & 41 \\
\hline \begin{tabular}{|l}
$216-218$ \\
\end{tabular} & 526.12 & \begin{tabular}{|l|}
1471 \\
\end{tabular} & 0.00 & \begin{tabular}{|l|}
0.00 \\
\end{tabular} & 0.00 & \begin{tabular}{|l|}
0.00 \\
\end{tabular} & \begin{tabular}{|l|}
0.00 \\
\end{tabular} & \begin{tabular}{|l|}
28.00 \\
\end{tabular} & 0.00 & \begin{tabular}{l|l}
0.00 \\
\end{tabular} & 0.00 & \begin{tabular}{|l|}
0.00 \\
\end{tabular} & \begin{tabular}{|l|}
0.00 \\
\end{tabular} & \begin{tabular}{|l|}
0.00 \\
\end{tabular} & \begin{tabular}{|l|}
0.00 \\
\end{tabular} & \begin{tabular}{|l|}
0.00 \\
\end{tabular} & \begin{tabular}{|l|}
0.00 \\
\end{tabular} & 4.00 & \begin{tabular}{|l|}
0.00 \\
\end{tabular} & 25 \\
\hline $220-222$ & 539.45 & 1458 & 0.00 & \begin{tabular}{|l|}
0.00 \\
\end{tabular} & 0.00 & \begin{tabular}{|l|}
0.00 \\
\end{tabular} & \begin{tabular}{|l|l|}
0.00 \\
\end{tabular} & 20.00 & 0.00 & 0.00 & 20.00 & \begin{tabular}{|l|}
0.00 \\
\end{tabular} & $\begin{array}{l}0.00 \\
\end{array}$ & \begin{tabular}{|l|}
0.00 \\
\end{tabular} & \begin{tabular}{|l|}
0.00 \\
\end{tabular} & \begin{tabular}{|l|}
0.00 \\
\end{tabular} & \begin{tabular}{|l|}
0.00 \\
\end{tabular} & 6.67 & \begin{tabular}{|l|}
0.00 \\
\end{tabular} & 15 \\
\hline $224-226$ & 552.91 & 1444 & 7.69 & \begin{tabular}{|l|}
0.00 \\
\end{tabular} & 0.00 & $\begin{array}{l}0.00 \\
\end{array}$ & \begin{tabular}{|l|}
7.69 \\
\end{tabular} & \begin{tabular}{|l|}
7.69 \\
\end{tabular} & \begin{tabular}{c|}
0.00 \\
\end{tabular} & \begin{tabular}{l|l}
0.00 \\
\end{tabular} & 0.00 & \begin{tabular}{|l|}
0.00 \\
\end{tabular} & $\begin{array}{l}0.00 \\
\end{array}$ & \begin{tabular}{|l|}
7.69 \\
\end{tabular} & \begin{tabular}{|l|}
0.00 \\
\end{tabular} & \begin{tabular}{|l|}
0.00 \\
\end{tabular} & \begin{tabular}{|l|}
0.00 \\
\end{tabular} & 7.69 & \begin{tabular}{|l|}
0.00 \\
\end{tabular} & 13 \\
\hline
\end{tabular}




\begin{tabular}{|c|c|c|c|c|c|c|c|c|c|c|c|c|c|c|c|c|}
\hline Depth (cm) & Years (B.P.) & Age Model Year & 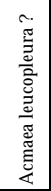 & 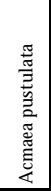 & 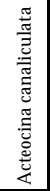 & $\begin{array}{l}\dot{\theta} \\
\tilde{0} \\
\frac{0}{2} \\
\frac{0}{0} \\
\frac{2}{4}\end{array}$ & $\begin{array}{l}\frac{\pi}{4} \\
\frac{4}{4} \\
\frac{\pi}{4} \\
\frac{8}{8} \\
\frac{8}{4}\end{array}$ & 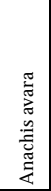 & $\begin{array}{l}\frac{0}{8} \\
\frac{8}{8} \\
\frac{0}{2} \\
\frac{8}{8} \\
\frac{5}{4} \\
\frac{6}{4}\end{array}$ & 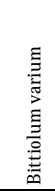 & $\begin{array}{l}\frac{\pi}{0} \\
\frac{0}{2} \\
\dot{t} \\
\frac{\pi}{5} \\
\overline{\bar{J}} \\
0\end{array}$ & 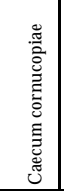 & 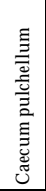 & 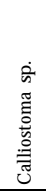 & 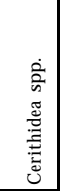 & 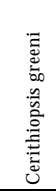 \\
\hline $0-2$ & 0.00 & 1997 & 0.75 & 0.00 & 0.00 & 0.00 & 0.00 & 0.00 & 1.50 & 2.26 & 0.00 & 0.75 & 1.50 & 0.00 & 0.00 & 0.00 \\
\hline $4-6$ & 2.69 & 1994 & 0.00 & 0.00 & 0.00 & 0.00 & 0.00 & 0.00 & 0.00 & 5.56 & 0.00 & 5.56 & 0.00 & 0.00 & 0.00 & 0.00 \\
\hline 8-10 & 9.24 & 1988 & 0.00 & 1.72 & 0.00 & 0.00 & 3.45 & 0.00 & 1.72 & 1.72 & 0.00 & 1.72 & 0.00 & 0.00 & 0.00 & 0.00 \\
\hline $12-14$ & 15.92 & 1981 & 0.00 & 0.00 & 0.00 & 0.00 & 4.00 & 0.00 & 0.00 & 4.00 & 0.00 & 0.00 & 0.00 & 0.00 & 0.00 & 0.00 \\
\hline $16-18$ & 22.72 & 1974 & 0.00 & 4.76 & 0.00 & 0.00 & 0.95 & 0.95 & 0.00 & 0.95 & 0.00 & 0.95 & 0.95 & 0.00 & 0.00 & 0.00 \\
\hline $20-22$ & 29.65 & 1967 & 0.54 & 1.63 & 0.00 & 0.54 & 0.00 & 0.54 & 1.09 & 9.78 & 0.00 & 0.54 & 0.00 & 0.00 & 0.00 & 0.00 \\
\hline $24-26$ & 36.71 & 1960 & 1.52 & 0.00 & 0.00 & 0.00 & 0.00 & 0.00 & 1.52 & 18.18 & 0.00 & 0.00 & 0.00 & 0.00 & 0.00 & 0.76 \\
\hline $28-30$ & 43.90 & 1953 & 1.98 & 0.00 & 0.00 & 0.00 & 0.00 & 1.98 & 0.00 & 10.89 & 0.00 & 6.93 & 0.00 & 0.00 & 0.00 & 0.00 \\
\hline $32-34$ & 51.22 & 1946 & 0.00 & 0.00 & 0.00 & 0.00 & 0.00 & 0.00 & 0.00 & 28.00 & 0.00 & 0.00 & 0.00 & 0.00 & 0.00 & 0.00 \\
\hline $36-38$ & 58.66 & 1938 & 0.00 & 0.00 & 0.00 & 0.00 & 0.00 & 0.00 & 0.00 & 20.37 & 0.00 & 0.00 & 0.00 & 0.00 & 0.00 & 1.85 \\
\hline $40-42$ & 66.23 & 1931 & 0.00 & 0.00 & 0.00 & 0.00 & 1.41 & 1.41 & 0.00 & 15.49 & 0.00 & 0.00 & 0.00 & 0.00 & 0.00 & 2.82 \\
\hline $44-46$ & 73.93 & 1923 & 0.00 & 0.00 & 0.00 & 0.00 & 0.00 & 0.00 & 0.00 & 17.39 & 0.00 & 0.00 & 0.00 & 0.00 & 0.00 & 4.35 \\
\hline $48-50$ & 81.76 & 1915 & 0.00 & 0.00 & 1.27 & 0.00 & 1.27 & 0.00 & 0.00 & 24.05 & 0.00 & 1.27 & 0.00 & 0.00 & 0.00 & 2.53 \\
\hline $52-54$ & 89.72 & 1907 & 0.00 & 0.00 & 0.00 & 0.00 & 0.00 & 0.00 & 0.00 & 14.58 & 0.00 & 0.00 & 0.00 & 0.00 & 0.00 & 0.00 \\
\hline $56-58$ & 97.80 & 1899 & 0.00 & 0.00 & 0.00 & 0.00 & 0.00 & 0.00 & 0.00 & 11.76 & 0.00 & 0.00 & 0.00 & 0.00 & 0.00 & 0.00 \\
\hline 60-62 & 106.01 & 1891 & 0.00 & 0.00 & 0.00 & 0.00 & 0.00 & 0.00 & 0.00 & 25.00 & 0.00 & 0.00 & 0.00 & 0.00 & 0.00 & 0.00 \\
\hline 64-66 & 114.35 & 1883 & 0.00 & 0.00 & 0.00 & 0.00 & 0.00 & 0.00 & 0.00 & 0.00 & 0.00 & 0.00 & 0.00 & 0.00 & 0.00 & 0.00 \\
\hline 68-70 & 122.82 & 1874 & 0.00 & 0.00 & 0.00 & 0.00 & 0.00 & 0.00 & 0.00 & 14.29 & 0.00 & 0.00 & 0.00 & 0.00 & 0.00 & 0.00 \\
\hline $72-74$ & 131.42 & 1866 & 0.56 & 0.00 & 0.00 & 0.00 & 0.00 & 1.69 & 0.00 & 7.30 & 0.00 & 0.56 & 0.00 & 0.00 & 0.00 & 1.12 \\
\hline 76-78 & 140.14 & 1857 & 2.22 & 0.00 & 0.00 & 0.00 & 0.00 & 0.00 & 0.00 & 6.67 & 0.00 & 3.33 & 0.00 & 0.00 & 0.00 & 0.00 \\
\hline $80-82$ & 148.99 & 1848 & 0.00 & 0.00 & 0.00 & 0.00 & 0.00 & 0.00 & 0.00 & 52.38 & 0.00 & 1.19 & 0.00 & 0.00 & 0.00 & 0.00 \\
\hline $84-86$ & 157.97 & 1839 & 0.00 & 0.00 & 0.00 & 0.00 & 0.00 & 0.00 & 0.00 & 89.47 & 0.00 & 0.00 & 0.00 & 0.00 & 0.00 & 0.00 \\
\hline 88-90 & 167.08 & 1830 & 0.00 & 0.00 & 0.00 & 0.00 & 0.00 & 0.00 & 0.00 & 48.39 & 0.00 & 0.00 & 0.00 & 0.00 & 0.00 & 0.00 \\
\hline $92-94$ & 176.32 & 1821 & 3.45 & 0.00 & 0.00 & 0.00 & 0.00 & 0.00 & 0.00 & 20.69 & 0.00 & 0.00 & 0.00 & 0.00 & 0.00 & 0.00 \\
\hline $96-98$ & 185.68 & 1811 & 0.00 & 0.00 & 0.00 & 0.00 & 0.00 & 0.00 & 0.00 & 8.70 & 0.00 & 4.35 & 0.00 & 0.00 & 0.00 & 13.04 \\
\hline $100-102$ & 195.17 & 1802 & 0.00 & 0.00 & 0.00 & 0.00 & 0.00 & 0.00 & 0.00 & 25.00 & 0.00 & 11.84 & 0.00 & 0.00 & 0.00 & 1.32 \\
\hline 104-106 & 204.79 & 1792 & 0.00 & 0.00 & 0.00 & 0.00 & 0.00 & 0.00 & 0.00 & 23.89 & 0.00 & 10.62 & 0.00 & 0.00 & 0.00 & 6.19 \\
\hline $108-110$ & 214.54 & 1782 & 0.00 & 0.00 & 0.00 & 0.00 & 0.00 & 0.00 & 0.00 & 42.11 & 0.00 & 0.00 & 0.00 & 0.00 & 0.00 & 0.00 \\
\hline $112-114$ & 224.42 & 1773 & 0.00 & 0.00 & 0.00 & 0.00 & 0.00 & 0.00 & 0.00 & 0.00 & 0.00 & 0.00 & 0.00 & 0.00 & 0.00 & 0.00 \\
\hline 116-118 & 234.42 & 1763 & 0.00 & 0.00 & 0.00 & 0.00 & 0.00 & 0.00 & 0.00 & 20.00 & 0.00 & 0.00 & 0.00 & 0.00 & 0.00 & 0.00 \\
\hline $120-122$ & 244.55 & 1752 & 0.00 & 0.00 & 0.00 & 0.00 & 0.00 & 0.00 & 0.00 & 67.65 & 0.00 & 4.90 & 0.00 & 0.00 & 0.00 & 0.00 \\
\hline $124-126$ & 254.81 & 1742 & 0.00 & 0.00 & 0.00 & 0.00 & 0.00 & 0.00 & 0.00 & 52.63 & 0.00 & 0.00 & 0.00 & 0.00 & 0.00 & 0.00 \\
\hline $128-130$ & 265.20 & 1732 & 0.00 & 0.00 & 0.00 & 0.00 & 0.00 & 0.00 & 0.00 & 44.00 & 0.00 & 0.00 & 0.00 & 0.00 & 0.00 & 0.00 \\
\hline $132-134$ & 275.72 & 1721 & 0.00 & 0.00 & 0.00 & 0.00 & 0.00 & 0.00 & 0.00 & 17.07 & 0.00 & 0.00 & 0.00 & 0.00 & 4.88 & 0.00 \\
\hline 136-138 & 286.36 & 1711 & 0.00 & 0.00 & 0.00 & 0.00 & 0.00 & 0.00 & 0.00 & 11.11 & 0.00 & 0.00 & 0.00 & 0.00 & 11.11 & 0.00 \\
\hline 140-142 & 297.13 & 1700 & 0.00 & 0.00 & 0.00 & 0.00 & 0.00 & 0.00 & 0.00 & 0.00 & 0.00 & 0.00 & 0.00 & 0.00 & 0.00 & 0.00 \\
\hline $144-146$ & 308.03 & 1689 & 0.00 & 0.00 & 0.00 & 0.00 & 0.00 & 0.00 & 0.00 & 6.25 & 0.00 & 6.25 & 0.00 & 0.00 & 0.00 & 0.00 \\
\hline $148-150$ & 319.06 & 1678 & 0.00 & 0.00 & 0.00 & 0.00 & 0.00 & 0.00 & 0.00 & 22.73 & 0.00 & 9.09 & 4.55 & 0.00 & 0.00 & 0.00 \\
\hline $152-154$ & 330.22 & 1667 & 0.00 & 0.00 & 0.00 & 0.00 & 0.00 & 0.00 & 0.00 & 11.43 & 0.00 & 5.71 & 0.00 & 2.86 & 0.00 & 0.00 \\
\hline 156-158 & 341.50 & 1655 & 0.00 & 0.00 & 0.00 & 0.00 & 0.00 & 0.00 & 0.00 & 27.78 & 0.00 & 0.00 & 0.00 & 0.00 & 0.00 & 2.78 \\
\hline $160-162$ & 350.05 & 1647 & 1.28 & 0.00 & 0.00 & 0.00 & 0.00 & 0.00 & 0.00 & 52.56 & 0.00 & 0.00 & 0.00 & 0.00 & 0.00 & 2.56 \\
\hline 164-166 & 364.45 & 1633 & 0.00 & 0.00 & 0.00 & 0.00 & 0.00 & 0.00 & 0.00 & 57.45 & 0.00 & 0.00 & 0.00 & 0.00 & 0.00 & 0.00 \\
\hline 168-170 & 376.12 & 1621 & 0.00 & 0.00 & 0.00 & 0.00 & 0.00 & 0.00 & 0.00 & 36.36 & 0.00 & 0.00 & 0.00 & 0.00 & 0.00 & 4.55 \\
\hline 172-174 & 387.92 & 1609 & 0.00 & 0.00 & 0.00 & 0.00 & 0.00 & 0.00 & 0.00 & 14.29 & 0.00 & 7.14 & 0.00 & 0.00 & 0.00 & 3.57 \\
\hline \begin{tabular}{|l|}
$176-178$ \\
\end{tabular} & 399.84 & 1597 & 0.00 & 0.00 & 0.00 & 0.00 & 0.00 & 0.00 & 0.00 & 5.26 & 0.00 & 0.00 & 0.00 & 5.26 & 0.00 & 0.00 \\
\hline \begin{tabular}{|l|}
$180-182$ \\
\end{tabular} & 411.89 & 1585 & 0.00 & 0.00 & 0.00 & 0.00 & 0.00 & 0.00 & 0.00 & 10.00 & 0.00 & 0.00 & 0.00 & 0.00 & 0.00 & 0.00 \\
\hline \begin{tabular}{|l|}
$184-186$ \\
\end{tabular} & 424.07 & 1573 & 0.00 & 0.00 & 0.00 & 0.00 & 0.00 & 0.00 & 0.00 & 9.09 & 0.00 & 0.00 & 0.00 & 0.00 & 0.00 & 0.00 \\
\hline 188-190 & 436.38 & 1561 & 0.00 & 0.00 & 0.00 & 0.00 & 0.00 & 0.00 & 0.00 & 7.41 & 0.00 & 3.70 & 0.00 & 0.00 & 0.00 & 7.41 \\
\hline \begin{tabular}{|l|}
$192-194$ \\
\end{tabular} & 448.82 & 1548 & 2.50 & 0.00 & 0.00 & 0.00 & 0.00 & 0.00 & 2.50 & 0.00 & 0.00 & 0.00 & 0.00 & 0.00 & 0.00 & 5.00 \\
\hline \begin{tabular}{|l|}
$196-198$ \\
\end{tabular} & 461.38 & 1536 & 0.00 & 0.00 & 0.00 & 0.00 & 0.00 & 0.00 & 0.00 & 0.00 & 0.00 & 0.00 & 0.00 & 0.00 & 0.00 & 0.00 \\
\hline $200-202$ & 474.07 & 1523 & 0.00 & 0.00 & 0.00 & 0.00 & 0.00 & 0.00 & 0.00 & 0.00 & 0.00 & 0.00 & 0.00 & 0.00 & 0.00 & 9.09 \\
\hline 204-206 & 486.89 & 1510 & 0.00 & 0.00 & 0.00 & 0.00 & 0.00 & 0.00 & 0.00 & 0.00 & 11.11 & 0.00 & 0.00 & 0.00 & 0.00 & 0.00 \\
\hline 208-210 & 499.84 & 1497 & 0.00 & 0.00 & 0.00 & 0.00 & 0.00 & 0.00 & 0.00 & 0.00 & 0.00 & 0.00 & 0.00 & 0.00 & 0.00 & 0.00 \\
\hline 212-214 & 512.92 & 1484 & 7.32 & 0.00 & 0.00 & 0.00 & 0.00 & 0.00 & 0.00 & 2.44 & 0.00 & 2.44 & 0.00 & 0.00 & 0.00 & 0.00 \\
\hline 216-218 & 526.12 & 1471 & 0.00 & 0.00 & 0.00 & 0.00 & 0.00 & 0.00 & 4.00 & 0.00 & 0.00 & $\begin{array}{ll}0.00 \\
\end{array}$ & 0.00 & 0.00 & 0.00 & 0.00 \\
\hline $220-222$ & 539.45 & 1458 & 0.00 & 0.00 & 0.00 & 0.00 & 0.00 & 0.00 & 0.00 & 6.67 & 0.00 & 0.00 & 0.00 & 0.00 & 0.00 & 0.00 \\
\hline $224-226$ & 552.91 & 1444 & 0.00 & 0.00 & 0.00 & 0.00 & 0.00 & 0.00 & 0.00 & 0.00 & 0.00 & 0.00 & 0.00 & 0.00 & 0.00 & 0.00 \\
\hline
\end{tabular}




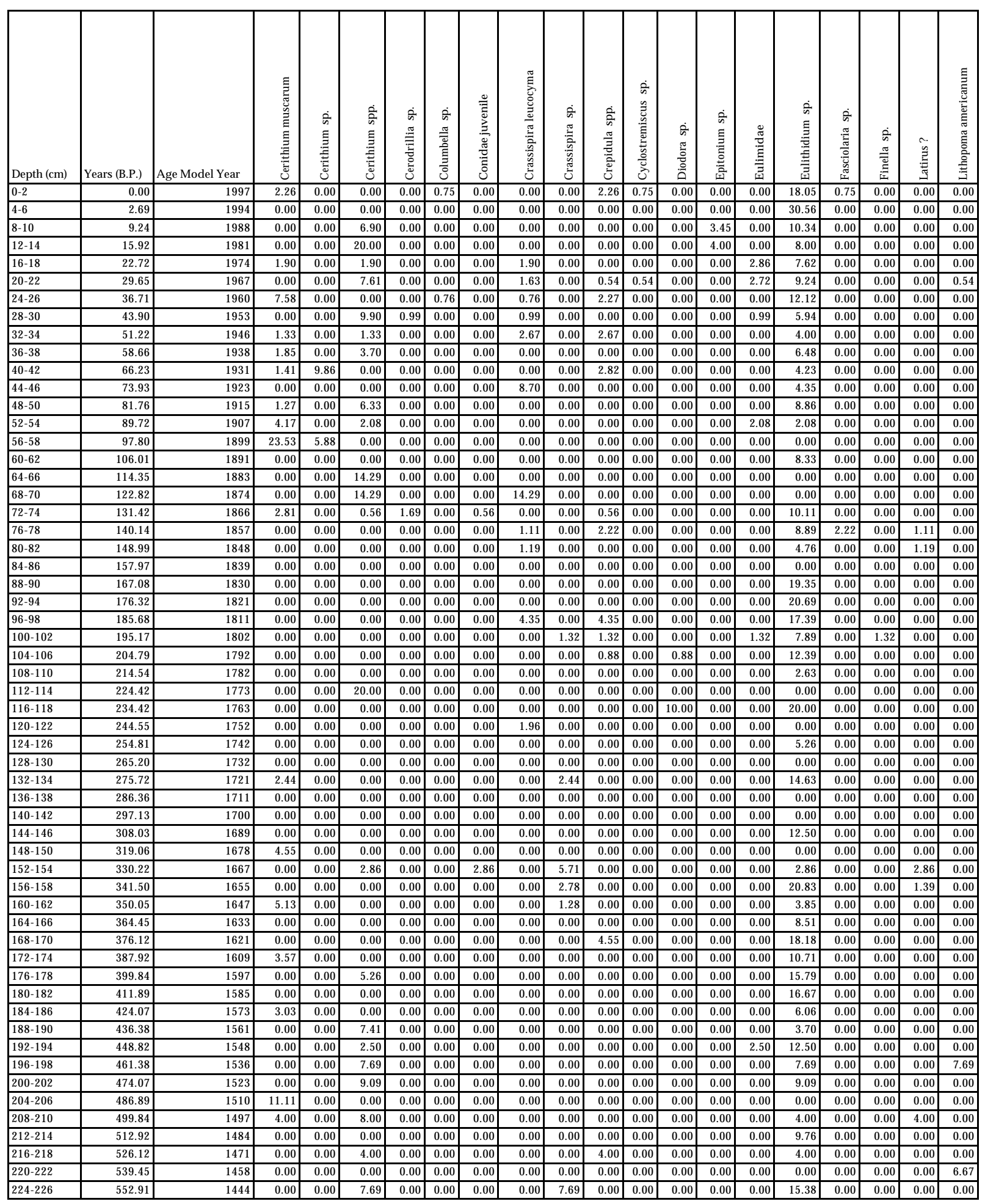




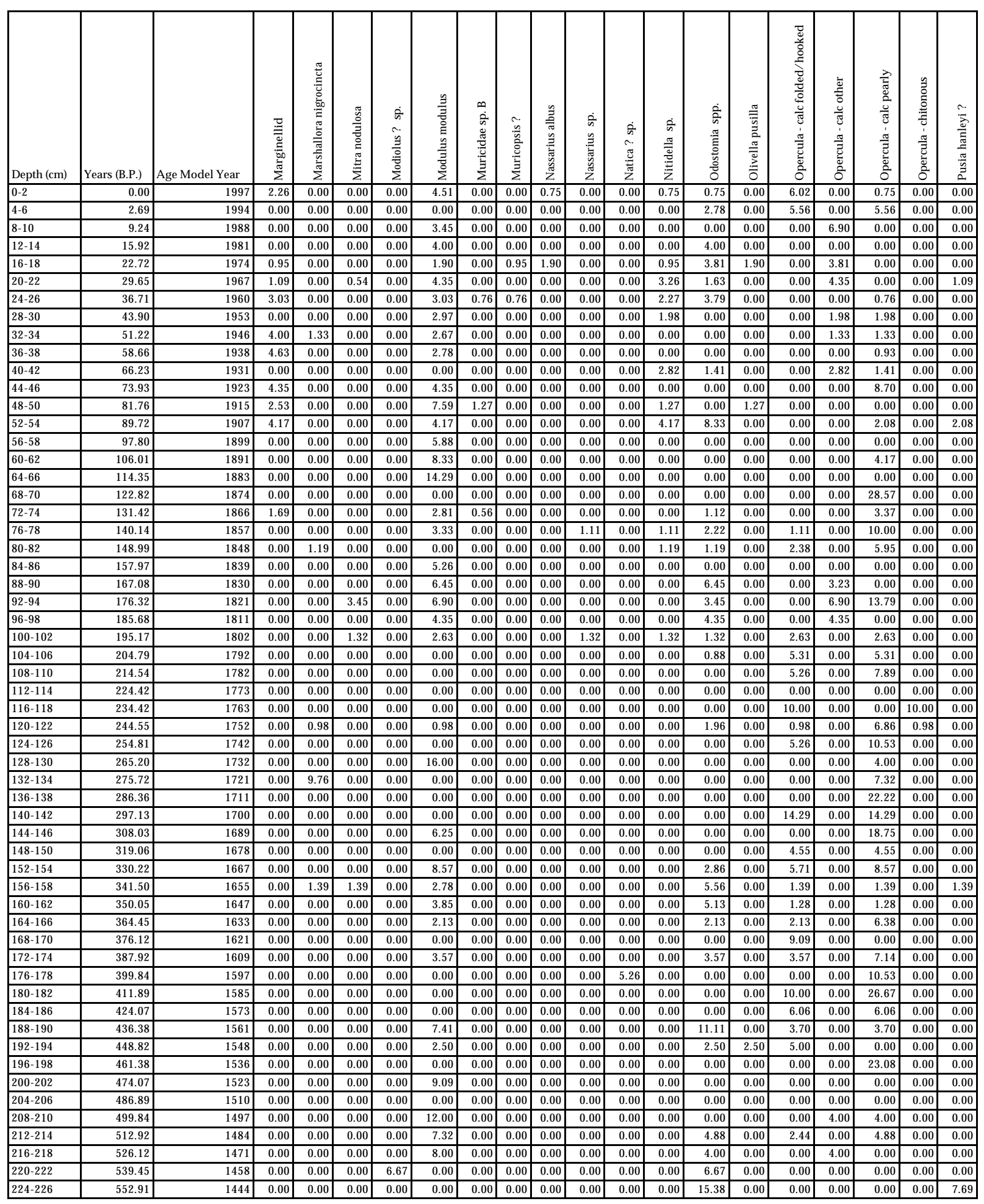




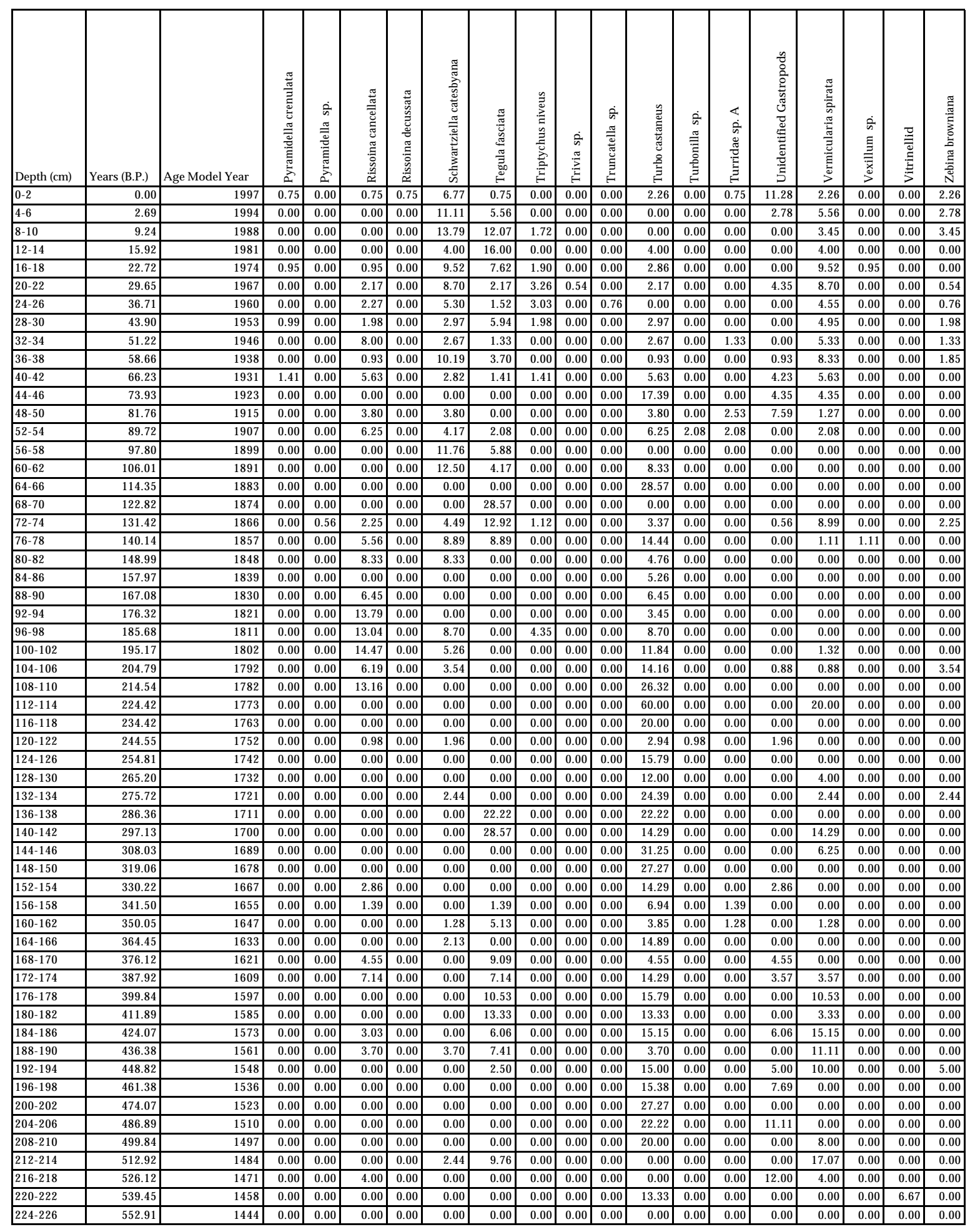





\begin{tabular}{|c|c|c|c|c|c|c|c|c|c|c|c|c|c|c|c|c|c|c|c|c|c|}
\hline Depth (cm) & Years (B.P.) & Age Model Year & 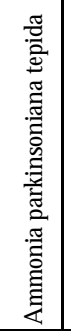 & 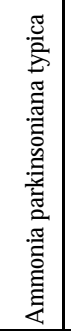 & 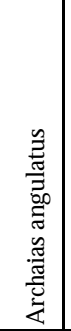 & 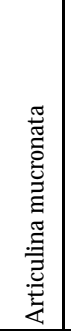 & 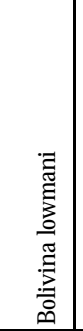 & 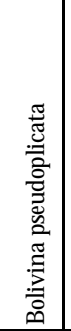 & 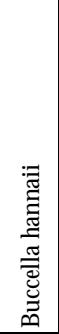 & $\begin{array}{l}\frac{0}{0} \\
\frac{y}{0} \\
\frac{0}{0} \\
\frac{0}{0} \\
\frac{0}{0}\end{array}$ & 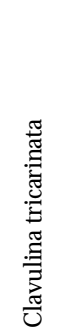 & 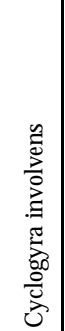 & 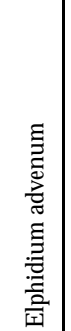 & 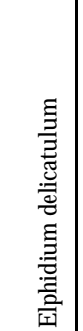 & 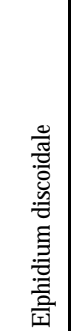 & 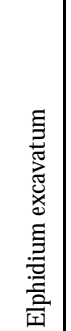 & 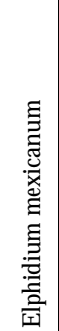 & 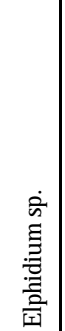 & 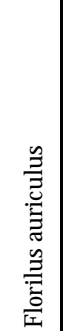 & 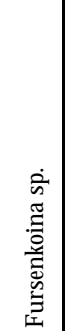 & 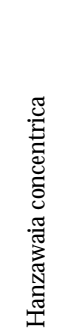 \\
\hline 116-118 & 234.42 & 1763 & 0.00 & 0.00 & 0.00 & 0.00 & 0.80 & 0.00 & 0.00 & 0.00 & 0.00 & 0.00 & 0.00 & 54.18 & 0.40 & 0.00 & 0.00 & 0.00 & 4.78 & 1.20 & 0.00 \\
\hline $120-122$ & 244.55 & 1752 & 0.00 & 0.00 & 0.31 & 0.00 & 0.93 & 0.00 & 3.43 & 0.00 & 0.00 & 0.31 & 0.00 & 38.01 & 0.00 & 0.00 & 0.00 & 0.00 & 1.25 & 0.31 & 0.00 \\
\hline $124-126$ & 254.81 & 1742 & 0.00 & 0.00 & 0.33 & 1.99 & 2.99 & 1.00 & 3.32 & 0.00 & 0.00 & 0.00 & 0.00 & 39.53 & 0.33 & 0.00 & 0.33 & 0.00 & 0.00 & 1.66 & 0.00 \\
\hline $128-130$ & 265.20 & 1732 & 0.00 & 0.00 & 0.61 & 3.34 & 0.61 & 0.00 & 5.78 & 0.00 & 0.00 & 0.00 & 1.22 & 20.36 & 0.61 & 0.00 & 0.30 & 0.00 & 0.61 & 0.00 & 0.00 \\
\hline 132-134 & 275.72 & 1721 & 0.00 & 2.57 & 5.15 & 5.51 & 0.00 & 0.00 & 0.00 & 0.00 & 0.74 & 0.00 & 0.00 & 31.62 & 0.00 & 0.00 & 1.47 & 0.00 & 0.37 & 0.00 & 0.00 \\
\hline 136-138 & 286.36 & 1711 & 0.00 & 9.20 & 4.60 & 0.00 & 0.00 & 0.00 & 0.00 & 0.00 & 0.00 & 0.00 & 9.20 & 31.03 & 10.92 & 0.00 & 4.02 & 0.00 & 1.72 & 0.00 & 0.00 \\
\hline $140-142$ & 297.13 & 1700 & 0.00 & 4.88 & 3.66 & 0.00 & 0.00 & 0.00 & 0.00 & 0.00 & 2.44 & 0.00 & 4.27 & 40.24 & 6.10 & 0.00 & 0.61 & 0.00 & 5.49 & 0.00 & 0.00 \\
\hline \begin{tabular}{|l|}
$144-146$ \\
\end{tabular} & 308.03 & 1689 & 0.00 & 0.32 & 0.63 & 0.32 & 0.00 & 0.00 & 0.00 & 0.00 & 0.00 & 0.00 & 0.63 & 54.29 & 0.63 & 0.00 & 0.63 & 0.00 & 5.71 & 0.00 & 0.00 \\
\hline $148-150$ & 319.06 & 1678 & 0.00 & 0.00 & 0.35 & 8.36 & 0.00 & 0.00 & 0.00 & 0.00 & 0.00 & 0.00 & 0.35 & 21.25 & 0.00 & 0.00 & 0.35 & 0.00 & 2.79 & 0.00 & 0.00 \\
\hline \begin{tabular}{|l|}
$152-154$ \\
\end{tabular} & 330.22 & 1667 & $\begin{array}{l}0.00 \\
\end{array}$ & 0.00 & 0.00 & 0.63 & 0.00 & 0.00 & 0.00 & 0.00 & 0.00 & 0.00 & 0.00 & 33.02 & 0.00 & 0.00 & 0.31 & 0.00 & 4.40 & 0.00 & 0.31 \\
\hline \begin{tabular}{|l}
$156-158$ \\
\end{tabular} & 341.50 & 1655 & \begin{tabular}{l|l}
0.00 \\
\end{tabular} & 0.00 & \begin{tabular}{l|}
1.21 \\
\end{tabular} & \begin{tabular}{l|}
1.21 \\
\end{tabular} & 0.00 & 0.00 & 0.00 & 0.00 & 0.00 & 0.00 & 0.00 & 18.13 & 0.30 & 0.00 & 0.00 & 0.00 & $\begin{array}{l}2.72 \\
\end{array}$ & 0.00 & 0.00 \\
\hline $160-162$ & 350.05 & 1647 & 0.00 & 0.00 & $\begin{array}{l}0.31 \\
\end{array}$ & $\begin{array}{l}0.31 \\
\end{array}$ & 0.00 & 0.00 & 0.00 & 0.00 & 0.00 & 0.00 & 0.00 & $\begin{array}{l}30.72 \\
\end{array}$ & 0.00 & 0.00 & 0.31 & 0.00 & 3.45 & 0.00 & 0.00 \\
\hline \begin{tabular}{|l|}
$164-166$ \\
\end{tabular} & 364.45 & 1633 & 0.00 & 0.00 & 0.00 & 0.00 & 0.00 & 0.00 & 0.00 & 0.00 & 0.00 & 0.00 & 1.32 & 54.28 & 0.99 & 0.00 & 0.00 & 0.00 & $\begin{array}{l}0.99 \\
\end{array}$ & 0.00 & 0.00 \\
\hline \begin{tabular}{|l|}
$168-170$ \\
\end{tabular} & 376.12 & 1621 & 0.00 & 0.00 & 0.00 & 0.00 & 0.00 & 0.00 & 0.00 & 0.00 & 0.00 & 0.00 & 0.65 & 51.46 & 0.65 & 0.00 & 0.00 & 0.00 & $\begin{array}{ll}4.53 \\
\end{array}$ & 0.00 & 0.00 \\
\hline \begin{tabular}{|l|}
$172-174$ \\
\end{tabular} & 387.92 & 1609 & 0.00 & 0.00 & 0.00 & 0.33 & 0.00 & 0.00 & 0.00 & 0.00 & 0.00 & 0.00 & 0.00 & $\begin{array}{l}62.46 \\
\end{array}$ & 1.66 & 0.00 & 0.00 & 0.00 & $\begin{array}{l}7.97 \\
\end{array}$ & 0.00 & 0.00 \\
\hline \begin{tabular}{|l}
$176-178$ \\
\end{tabular} & 399.84 & 1597 & $\begin{array}{l}0.00 \\
\end{array}$ & 0.00 & 0.00 & 0.00 & \begin{tabular}{|l|}
15.73 \\
\end{tabular} & 3.15 & 0.00 & 0.00 & 0.00 & 0.00 & 0.00 & 66.43 & 2.10 & 0.00 & 0.00 & 0.00 & 4.55 & 0.00 & 0.70 \\
\hline \begin{tabular}{|l|}
$180-182$ \\
\end{tabular} & 411.89 & 1585 & 0.00 & 0.00 & 0.31 & 0.00 & \begin{tabular}{|c|}
16.51 \\
\end{tabular} & 1.22 & 0.00 & 0.31 & 0.00 & 0.00 & 1.22 & 63.30 & 0.61 & 0.00 & 0.61 & 0.00 & 4.28 & 0.31 & 0.00 \\
\hline \begin{tabular}{|l|}
$184-186$ \\
\end{tabular} & 424.07 & 1573 & 0.00 & 0.85 & $\begin{array}{l}0.57 \\
\end{array}$ & 0.00 & 4.53 & 0.85 & 0.00 & 0.00 & 0.00 & 0.00 & 1.98 & $\begin{array}{l}63.17 \\
\end{array}$ & 4.25 & 0.00 & 0.28 & 0.00 & 3.97 & 0.85 & 0.00 \\
\hline \begin{tabular}{|l|}
$188-190$ \\
\end{tabular} & 436.38 & 1561 & 0.00 & 0.30 & \begin{tabular}{|l|}
1.81 \\
\end{tabular} & 0.30 & 0.00 & 0.90 & 0.00 & 0.00 & 0.00 & 0.00 & $\begin{array}{l}1.51 \\
\end{array}$ & 26.20 & 7.23 & 0.00 & 0.30 & 0.00 & 0.90 & 0.00 & 0.00 \\
\hline \begin{tabular}{|l|}
$192-194$ \\
\end{tabular} & 448.82 & 1548 & 0.00 & 2.57 & 2.57 & 0.00 & \begin{tabular}{|l|}
1.93 \\
\end{tabular} & 0.96 & 0.00 & 0.00 & 0.00 & 0.00 & \begin{tabular}{|c|}
9.97 \\
\end{tabular} & 36.33 & 15.43 & 0.00 & 0.00 & 0.00 & 0.00 & 0.00 & 0.00 \\
\hline \begin{tabular}{|l|}
$196-198$ \\
\end{tabular} & 461.38 & 1536 & 0.00 & 0.00 & 1.46 & 0.00 & 4.09 & 0.88 & 0.00 & 0.00 & 0.00 & 0.00 & 4.97 & 56.73 & $\begin{array}{r}6.14 \\
\end{array}$ & 0.00 & 0.58 & 0.00 & 2.92 & 0.58 & 0.00 \\
\hline $200-202$ & 474.07 & 1523 & 0.00 & 0.00 & 0.00 & 0.00 & 3.18 & 1.27 & 0.00 & 0.00 & 0.00 & 0.00 & 4.14 & $\begin{array}{l}62.42 \\
\end{array}$ & 6.05 & 2.55 & 0.00 & 0.00 & 1.91 & 0.96 & 0.00 \\
\hline \begin{tabular}{|l|}
$204-206$ \\
\end{tabular} & 486.89 & 1510 & 0.00 & 0.30 & 0.30 & 0.00 & 2.11 & 0.60 & 0.00 & 0.00 & 0.00 & 0.00 & 4.22 & 71.99 & 8.13 & 0.00 & 0.90 & 0.00 & 3.01 & 0.00 & 0.00 \\
\hline $208-210$ & 499.84 & 1497 & $\begin{array}{l}0.00 \\
\end{array}$ & 0.29 & 2.62 & 0.00 & 0.58 & 0.29 & 0.00 & 0.29 & 0.29 & 0.00 & 4.65 & \begin{tabular}{|l|}
44.77 \\
\end{tabular} & 18.31 & 0.00 & 0.00 & 0.00 & $\begin{array}{l}0.87 \\
\end{array}$ & 0.00 & 0.00 \\
\hline $212-214$ & 512.92 & 1484 & 0.00 & 1.72 & $\begin{array}{l}0.57 \\
\end{array}$ & 0.00 & 0.00 & 0.00 & 0.00 & 0.00 & 0.57 & 0.00 & 4.58 & \begin{tabular}{|l|}
45.27 \\
\end{tabular} & 15.19 & 0.00 & 0.00 & 0.00 & 2.29 & 0.29 & 0.00 \\
\hline $216-218$ & 526.12 & 1471 & 0.00 & 0.56 & 1.94 & 0.56 & 0.00 & 0.00 & 0.00 & 0.00 & 0.28 & 0.00 & 8.06 & 24.72 & 19.44 & 0.00 & 0.00 & 0.00 & 1.67 & 0.00 & 0.00 \\
\hline $220-222$ & 539.45 & 1458 & 0.00 & 0.00 & 0.96 & 0.32 & 0.32 & 0.32 & 0.00 & 0.00 & 0.96 & 0.00 & 0.00 & 37.90 & 17.20 & 0.00 & 0.00 & 0.00 & 0.00 & 0.32 & 0.00 \\
\hline $224-226$ & 552.91 & 1444 & 0.00 & 0.90 & 1.36 & 3.17 & 0.00 & 0.00 & 0.00 & 0.00 & 1.36 & 0.00 & 0.00 & \begin{tabular}{|l|}
13.12 \\
\end{tabular} & 6.79 & 0.00 & 0.00 & 0.00 & 0.90 & 0.00 & 0.00 \\
\hline
\end{tabular}




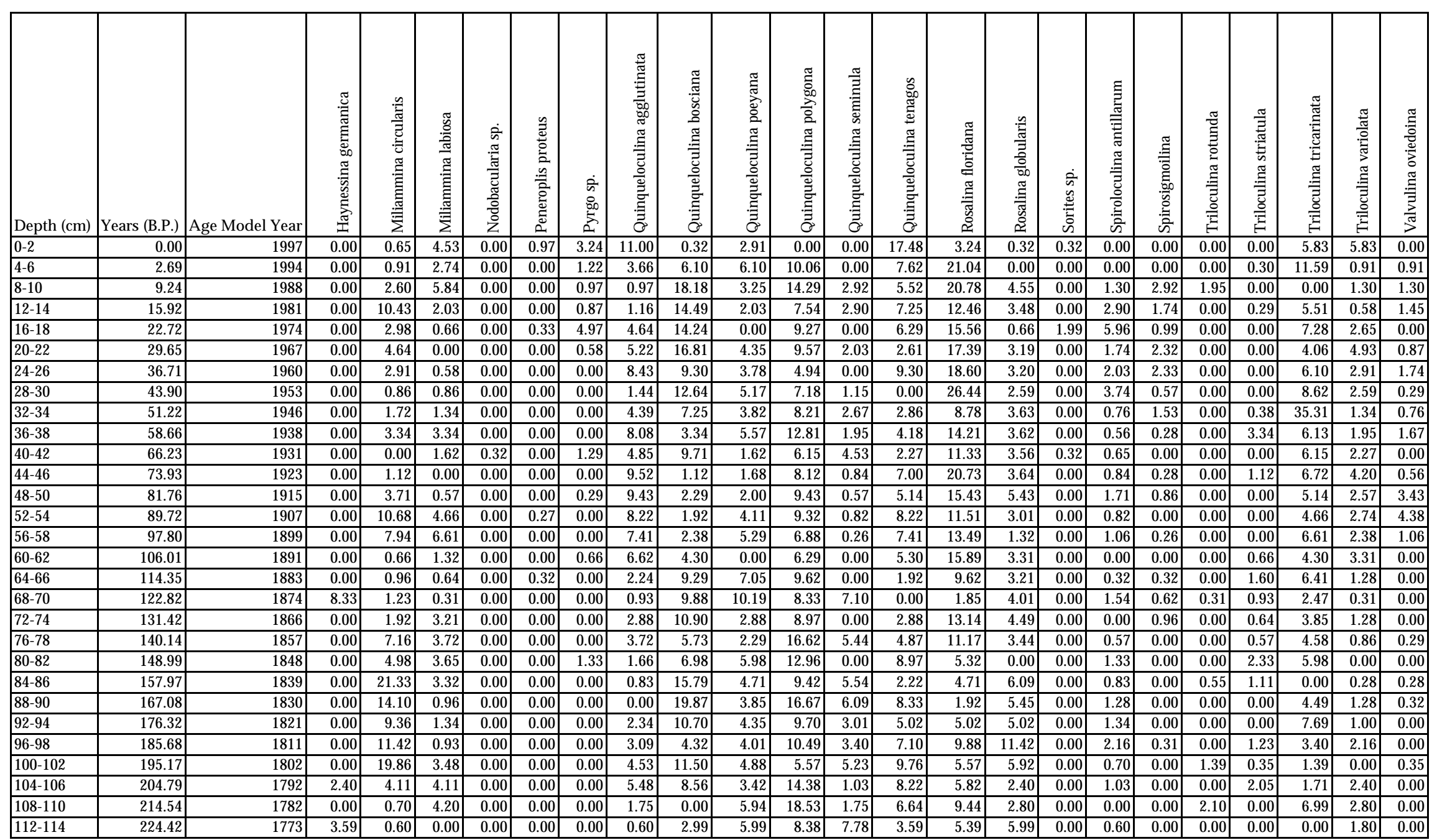




\begin{tabular}{|c|c|c|c|c|c|c|c|c|c|c|c|c|c|c|c|c|c|c|c|c|c|c|c|c|}
\hline Depth (cm) & Years (B.P.) & Age Model Year & 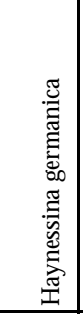 & 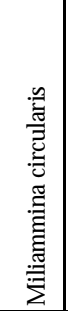 & 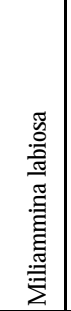 & $\begin{array}{l}\frac{2}{2} \\
\frac{0}{7} \\
\frac{0}{2} \\
\frac{8}{8} \\
\frac{0}{8} \\
z\end{array}$ & $\begin{array}{l}\frac{n}{\bar{y}} \\
\overline{0} \\
\frac{0}{0} \\
\frac{\bar{z}}{0} \\
0 \\
\overline{0} \\
0\end{array}$ & $\begin{array}{l}0 \\
0 \\
\text { o } \\
\sum_{0}^{2}\end{array}$ & 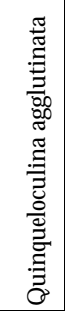 & 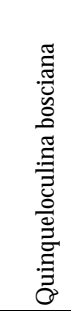 & 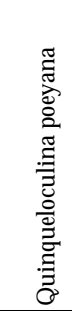 & 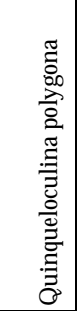 & 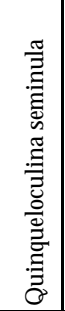 & 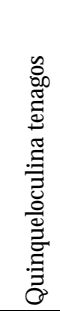 & 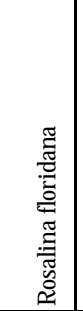 & $\begin{array}{l}\frac{n}{0} \\
\frac{\pi}{7} \\
\frac{0}{0} \\
0 \\
\pi \\
\frac{0}{0} \\
8 \\
8\end{array}$ & $\begin{array}{l}0 \\
\dot{0} \\
y \\
\dot{y} \\
\dot{0} \\
\dot{n}\end{array}$ & 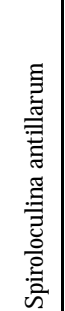 & $\begin{array}{l}\frac{\pi}{\bar{z}} \\
\overline{\bar{g}} \\
\bar{n} \\
\overline{8} \\
. \overline{0} \\
0\end{array}$ & 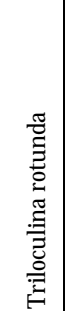 & 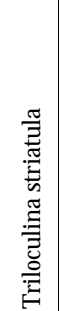 & 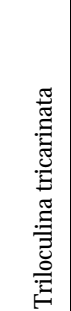 & 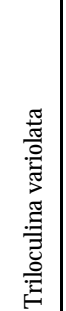 & 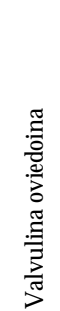 \\
\hline \begin{tabular}{|l|}
$116-118$ \\
\end{tabular} & 234.42 & 1763 & 0.00 & 0.80 & 0.40 & 0.00 & 0.00 & 0.00 & 0.00 & 17.93 & 3.19 & 1.59 & 2.39 & 1.59 & 1.20 & 5.98 & 0.00 & 0.00 & 0.00 & 0.00 & 0.80 & 2.79 & 0.00 & 0.00 \\
\hline $120-122$ & 244.55 & 1752 & 0.00 & 4.67 & 0.62 & 0.00 & 0.00 & 0.00 & 1.56 & 12.15 & 23.99 & 0.93 & 5.61 & 0.00 & 0.00 & 1.87 & 0.00 & 0.00 & 0.00 & 0.00 & 0.62 & 3.12 & 0.00 & 0.31 \\
\hline $124-126$ & $\begin{array}{l}254.81 \\
\end{array}$ & 1742 & 0.00 & 3.32 & 0.00 & 0.00 & 0.00 & 0.00 & 1.00 & 11.30 & 17.28 & 1.00 & 1.99 & 2.66 & 0.00 & 8.31 & 0.00 & 0.00 & 0.00 & 0.00 & 0.00 & 1.33 & 0.00 & 0.33 \\
\hline $128-130$ & 265.20 & 1732 & 0.00 & 4.26 & 3.65 & 0.61 & 0.00 & 0.00 & 5.47 & 8.21 & 9.42 & 15.81 & 0.91 & 7.60 & 0.00 & 1.52 & 0.00 & 0.61 & 0.00 & 0.30 & 0.00 & 8.21 & 0.00 & 0.00 \\
\hline $132-134$ & 275.72 & 1721 & 1.10 & 1.84 & 0.00 & 0.00 & 0.00 & 0.00 & 3.68 & 3.31 & 0.00 & 13.97 & 0.00 & 6.99 & 4.04 & 2.21 & 0.00 & 0.00 & 0.00 & 2.57 & 1.10 & 10.66 & \begin{tabular}{c|c|}
0.74 \\
\end{tabular} & 0.37 \\
\hline 136-138 & 286.36 & 1711 & 5.75 & 0.00 & 0.00 & 0.00 & 0.00 & 0.00 & 1.15 & 0.00 & 2.87 & 3.45 & 0.00 & 0.00 & 3.45 & 4.60 & 0.00 & 0.00 & 0.00 & 0.57 & 0.57 & 6.90 & 0.00 & 0.00 \\
\hline $140-142$ & 297.13 & 1700 & 12.20 & 0.00 & 0.00 & 0.00 & 0.00 & 0.00 & 0.00 & 1.83 & 3.05 & 1.83 & 0.61 & 0.00 & 1.22 & 4.27 & 0.00 & 0.00 & 0.00 & 0.00 & 0.00 & 6.71 & 0.00 & 0.61 \\
\hline 144-146 & 308.03 & 1689 & 0.00 & 0.00 & 0.00 & 0.00 & 0.00 & 0.00 & 1.27 & 5.71 & 4.76 & 5.40 & 5.08 & 0.63 & 2.54 & 6.67 & 0.00 & 0.32 & 0.00 & 0.32 & 0.00 & 3.81 & 0.32 & 0.00 \\
\hline \begin{tabular}{|l|}
$148-150$ \\
\end{tabular} & 319.06 & 1678 & 0.00 & 6.62 & 4.18 & 0.00 & 0.35 & 0.35 & 9.76 & 11.50 & 4.88 & 10.80 & 0.00 & 6.27 & 1.05 & 2.79 & 0.00 & 0.70 & 0.00 & 2.09 & 0.00 & 3.48 & $\begin{array}{l}1.74 \\
\end{array}$ & 0.00 \\
\hline \begin{tabular}{|l}
$152-154$ \\
\end{tabular} & 330.22 & 1667 & 0.00 & 2.20 & 1.26 & 0.00 & 0.00 & $\begin{array}{l}0.00 \\
\end{array}$ & 1.89 & 18.24 & 9.12 & 5.97 & 5.03 & 2.83 & 1.89 & 5.97 & 0.00 & 0.00 & $\begin{array}{l}1.57 \\
\end{array}$ & 0.31 & 1.57 & 2.83 & $\begin{array}{ll}0.63 \\
\end{array}$ & 0.00 \\
\hline \begin{tabular}{|l|}
$156-158$ \\
\end{tabular} & 341.50 & 1655 & 0.00 & 1.81 & 5.14 & 0.00 & 0.00 & 0.00 & 3.93 & 3.93 & 7.55 & 16.92 & 3.63 & 6.95 & 7.85 & 4.53 & 0.00 & 0.00 & 0.00 & 2.72 & 6.34 & 5.14 & 0.00 & 0.00 \\
\hline \begin{tabular}{|l|}
$160-162$ \\
\end{tabular} & 350.05 & 1647 & 0.00 & 0.94 & 2.19 & 0.00 & 0.00 & 0.00 & 2.19 & 4.08 & 10.03 & 15.99 & 5.33 & 5.64 & 6.58 & 4.08 & 0.00 & 0.31 & 0.00 & 0.00 & 0.00 & 6.58 & \begin{tabular}{l|l}
0.94 \\
\end{tabular} & 0.00 \\
\hline \begin{tabular}{|l|}
$164-166$ \\
\end{tabular} & 364.45 & 1633 & 0.00 & 0.99 & 4.28 & 0.00 & 0.00 & 0.00 & 1.32 & 0.00 & 15.46 & 10.86 & 0.00 & 3.95 & 1.32 & 0.66 & 0.00 & 0.00 & 0.00 & 0.00 & 2.30 & 0.99 & $\begin{array}{ll}0.33 \\
\end{array}$ & 0.00 \\
\hline \begin{tabular}{|l|}
$168-170$ \\
\end{tabular} & 376.12 & 621 & 0.00 & 0.32 & 0.00 & 0.00 & 0.00 & 0.00 & 0.00 & 0.97 & 24.60 & 0.00 & $\begin{array}{l}0.97 \\
\end{array}$ & 2.27 & 4.85 & 6.47 & 0.00 & 0.32 & 0.00 & 1.29 & 0.00 & 0.00 & 0.65 & 0.00 \\
\hline \begin{tabular}{|l|}
$172-174$ \\
\end{tabular} & 387.92 & 1609 & 0.00 & 0.00 & 0.00 & 0.00 & 0.00 & 0.00 & 0.33 & 3.32 & 7.31 & 2.66 & 0.33 & 2.99 & 2.33 & 4.98 & 0.00 & 0.33 & 0.00 & 0.00 & 0.00 & 2.99 & 0.00 & 0.00 \\
\hline \begin{tabular}{|l|}
$176-178$ \\
\end{tabular} & 399.84 & 1597 & 0.00 & 0.00 & 0.00 & 0.00 & 0.00 & 0.00 & 0.35 & 1.40 & 0.70 & 0.70 & 0.00 & 0.00 & 0.00 & 3.50 & 0.00 & 0.00 & 0.00 & 0.00 & 0.70 & 0.00 & 0.00 & 0.00 \\
\hline \begin{tabular}{|l}
$180-182$ \\
\end{tabular} & 411.89 & 1585 & 0.00 & 0.00 & 0.00 & 0.00 & 0.00 & 0.00 & 0.00 & 0.00 & 1.22 & 0.31 & 0.31 & 0.00 & 1.53 & 7.65 & 0.00 & 0.00 & 0.00 & 0.00 & 0.00 & 0.31 & 0.00 & 0.00 \\
\hline \begin{tabular}{|l|}
$184-186$ \\
\end{tabular} & 424.07 & 1573 & 0.00 & 0.00 & 0.00 & 0.00 & 0.00 & 0.00 & 1.42 & 0.28 & 2.27 & 1.98 & 0.00 & 0.28 & 1.13 & 5.67 & 0.00 & 0.57 & 0.00 & 0.28 & 0.00 & 4.53 & \begin{tabular}{l|l|}
0.28 \\
\end{tabular} & 0.00 \\
\hline \begin{tabular}{|l|}
$188-190$ \\
\end{tabular} & 436.38 & 1561 & 0.00 & 0.30 & 0.90 & 0.00 & 0.00 & 0.00 & $\begin{array}{l}1.81 \\
\end{array}$ & 0.90 & 6.63 & 13.25 & 3.61 & 8.13 & 3.31 & 3.31 & 0.00 & 0.00 & 0.00 & 0.00 & 0.00 & 15.06 & 2.71 & 0.60 \\
\hline \begin{tabular}{|l|}
$192-194$ \\
\end{tabular} & 448.82 & 1548 & 0.00 & 0.00 & 0.00 & 0.00 & 0.00 & 0.00 & 1.61 & 0.32 & 1.29 & 2.57 & 0.64 & 0.64 & 3.22 & 2.57 & 0.00 & 0.00 & 0.00 & 0.64 & 0.00 & 16.08 & \begin{tabular}{l|l|}
0.64 \\
\end{tabular} & 0.00 \\
\hline \begin{tabular}{|l|}
$196-198$ \\
\end{tabular} & 461.38 & 1536 & 0.00 & 0.00 & 0.00 & 0.00 & 0.00 & 0.00 & 0.00 & 0.88 & 3.80 & 0.58 & 0.00 & 1.17 & 3.22 & 7.02 & 0.00 & 0.00 & 0.00 & 0.29 & 0.00 & 4.39 & 0.29 & 0.00 \\
\hline $200-202$ & 474.07 & 1523 & 0.00 & 0.00 & 0.00 & 0.00 & 0.00 & 0.00 & 0.32 & 0.00 & 1.27 & 1.91 & 1.27 & 0.00 & 5.73 & 2.87 & 0.00 & 0.32 & 0.00 & 0.32 & 0.00 & 3.50 & 0.00 & 0.00 \\
\hline $204-206$ & 486.89 & 1510 & 0.00 & 0.00 & 0.00 & 0.00 & 0.00 & 0.00 & 0.00 & 0.00 & 2.11 & 1.20 & 0.90 & 1.81 & 0.00 & 0.00 & 0.00 & 0.00 & 0.00 & 0.00 & 0.00 & 2.41 & 0.00 & 0.00 \\
\hline $208-210$ & 499.84 & 1497 & 0.00 & 1.16 & 1.45 & 0.00 & 0.00 & 0.00 & 0.00 & 0.00 & 4.65 & 2.62 & 2.03 & 3.20 & 2.03 & 2.33 & 0.00 & 0.00 & 0.29 & 0.00 & 0.00 & 6.10 & 1.16 & 0.00 \\
\hline $212-214$ & 512.92 & 1484 & 0.00 & 2.01 & 1.15 & 0.00 & 0.00 & 0.00 & 1.43 & 0.00 & 4.58 & $\begin{array}{l}4.87 \\
\end{array}$ & 2.58 & 2.29 & 2.87 & 2.58 & 0.00 & 0.00 & 0.00 & 0.00 & 0.00 & 4.58 & $\begin{array}{l}0.57 \\
\end{array}$ & 0.00 \\
\hline $216-218$ & 526.12 & 1471 & 0.00 & 0.28 & 0.56 & 0.00 & 0.00 & 0.00 & 1.94 & 1.39 & 1.67 & 4.72 & 0.28 & 5.00 & 9.44 & 2.22 & 0.00 & 0.00 & 0.00 & 0.56 & 0.00 & 13.06 & $\begin{array}{l}1.67 \\
\end{array}$ & 0.00 \\
\hline $220-222$ & 539.45 & 1458 & 0.00 & 0.64 & 0.00 & 0.00 & 0.00 & 0.00 & 3.18 & 2.23 & 6.37 & 4.14 & 0.64 & 6.05 & 2.55 & 1.91 & 0.00 & 0.00 & 0.00 & 0.32 & 0.00 & 12.74 & $\begin{array}{l}0.96 \\
\end{array}$ & 0.00 \\
\hline $224-226$ & 552.91 & 1444 & 0.00 & 2.71 & 2.26 & 0.00 & 0.00 & $\begin{array}{l}0.00 \\
\end{array}$ & 2.26 & 1.81 & 4.98 & 9.95 & 4.07 & 9.50 & 17.65 & 3.62 & 0.00 & 0.00 & 0.00 & 0.00 & 2.26 & 8.14 & \begin{tabular}{|c|}
1.81 \\
\end{tabular} & 1.36 \\
\hline
\end{tabular}

